\title{
BOOLEAN ALGEBRA APPROXIMATIONS
}

\author{
KENNETH HARRIS AND ANTONIO MONTALBÁN
}

\begin{abstract}
Knight and Stob proved that every low4 Boolean algebra is $0^{(6)}$ isomorphic to a computable one. Furthermore, for $n=1,2,3,4$, every low $n$ Boolean algebra is $0^{(n+2)}$-isomorphic to a computable one. We show that this is not true for $n=5$ : there is a low 5 Boolean algebra that is not $0^{(7)}$-isomorphic to any computable Boolean algebra.

It is worth remarking that, because of the machinery developed, the proof uses at most a $0^{\prime \prime}$-priority argument. The technique used to construct this Boolean algebra is new and might be useful in other applications, such as to solve the low ${ }_{n}$ Boolean algebra problem either positively or negatively.
\end{abstract}

\section{INTRODUCTION}

Computable structures are one of the main objects of study of effective mathematics. Which mathematical structures have computable presentations is an active area of research in effective mathematics and computability theory. Related to this is the question of what kind of information can be encoded in the isomorphism type of a given structure. Downey and Jockusch DJ94 proved that every low Boolean algebra has a computable presentation. In other words, if the information encoded in the isomorphism type of a Boolean algebra is low, then there is no information in it at all. This result was extended by Thurber [Thu95] to low 2 Boolean algebras, and by Knight and Stob $\mathrm{KS} 00$ ] to $\mathrm{low}_{4}$.

The natural question to follow up with is whether every low ${ }_{n}$ Boolean algebra has a computable presentation. This problem, originally posed by Downey and Jockusch [DJ94, is still open. One problem here is that the combinatorics of the proofs get exponentially more complicated at each level. We will show that there are also new types of obstacles that appear for the first time at $n=5$. When $n=1,2,3,4$, it follows from the earlier results that every $\operatorname{low}_{n}$ Boolean algebra is isomorphic to a computable one via an isomorphism that is computable in $0^{(n+2)}$. (For $n=2$, the isomorphism found by Thurber is actually computable in $0^{\prime \prime \prime}$.) We construct a low 5 Boolean algebra that is not $0^{(5+2)}$-isomorphic to any computable one. Therefore, a proof that every low 5 Boolean algebra is isomorphic to a computable one would have to be, in essence, different from the known proofs for the lower cases.

An interesting feature of our proof is that it does not use more than an infinite injury construction. This is due to the new techniques developed, which are based

Received by the editors February 27, 2010 and, in revised form, September 5, 2012.

2010 Mathematics Subject Classification. Primary 03D45, 03 G05.

Key words and phrases. Boolean algebra, back-and-forth, low, approximation.

The second author was partially supported by NSF grant DMS-0901169, and by the AMS centennial fellowship. 
on the authors' work in [HM]. The main notion is that of an $n$ - $Z$-approximation of a Boolean algebra. We believe that this notion will be useful in a solution of the low problem, and more generally, in problems regarding degree spectra or relational spectra of Boolean algebras.

In Section 2, we briefly review the main definitions and results of [HM], which will suffice for the reader to understand the main ideas in our proof. In [HM], we studied the $n$-back-and-forth relations on Boolean algebras, providing general invariants for the back-and-forth equivalence classes of algebras. These invariants are constructed, for each $n$, from a finite set of special types we denote by $\mathbf{B F}_{n}$ and call the $n$-indecomposable back-and-forth types. (A Boolean algebra is $n$ indecomposable if for any way of expressing the algebra as a finite sum of subalgebras, at least one subalgebra is in the same $n$-back-and-forth class. See Definition 2.3.) We additionally provided, for each $n$, a finite set of Boolean algebra unary predicates $\mathrm{R}_{\alpha}$, one for each $\alpha \in \mathbf{B F}_{n}$, which are interdefinable with the sets of predicates used in DJ94, Thu95, KS00, to solve the low 4 Boolean algebra problem. The main property of our predicates is that given a Boolean algebra $\mathcal{B}$ and a set $Z \geq 0^{(n)}$, the following two statements are equivalent:

- The computably infinitary $\Pi_{n}^{c}$ diagram of $\mathcal{B}$ (to be defined later) is computable in $Z$;

- $\mathcal{B}$ and the finitely many relations $\mathrm{R}_{\alpha}(\mathcal{B})$ for $\alpha \in \mathbf{B F}_{n}$ are computable in $Z$.

This result motivates the following definition.

Definition 1.1. Given $n$ and a Boolean algebra $\mathcal{A}=(A, \vee, \wedge,-, 0,1)$, we let $\mathrm{B}_{n}(\mathcal{A})$ be the structure

$$
\left(A, \vee, \wedge,-, 0,1, \mathrm{R}_{\alpha}(\mathcal{A}): \alpha \in \mathbf{B F}_{n}\right)
$$

where $\mathrm{R}_{\alpha}(\mathcal{A})=\left\{a \in A: \mathcal{A} \models \mathrm{R}_{\alpha}(a)\right\}$. We say that $\mathcal{A}$ is $n$-Z-approximable if $\mathrm{B}_{n}(\mathcal{A})$ is computable in $Z$.

The main lemmas in DJ94, Thu95, KS00 say that for $n=1,2,3,4$, every $n$ - $Z^{\prime}$ approximable Boolean algebra has a copy that is $(n-1)-Z$-approximable. If $\mathcal{A}$ is a computable, or even a low ${ }_{n}$, Boolean algebra, then $\mathrm{B}_{n}(\mathcal{A})$ is $0^{(n)}$-computable. Conversely, if follows from [Mon, Theorem 3.1] that if $\mathrm{B}_{n}(\mathcal{A})$ is $0^{(n)}$-computable, then $\mathcal{A}$ has a low ${ }_{n}$ copy. (Using the notation from [Mon, we have that $\mathrm{B}_{n}(\mathcal{A})$ is the $n$th jump of the structure $\mathcal{A}$.) By [Mon, Theorem 3.5] the low $_{n}$ Boolean algebra question can be restated as follows.

Question 1. Does every $n$ - $Z^{(n)}$-approximable Boolean algebra have a $Z$-computable copy?

We will approximate $n$ - $Z$-approximable Boolean algebras by $Z$-computable sequences of finite labeled Boolean algebras. This type of approximation, which is the main concept of the paper, is introduced in Section 3 and applied in the subsequent sections.

The rest of the paper is dedicated to building a low 5 Boolean algebra that is not $0^{(7)}$-isomorphic to any computable Boolean algebra. In Section 5 we establish some lemmas used in the construction of the low 5 Boolean algebra in Section 6 . 


\section{BACKGROUND}

2.1. Preliminaries. Let $L$ be a computable language. We will be considering $\Sigma_{n}$ and $\Pi_{n}$ formulas in $L_{\omega_{1} \omega}$ and also computable $\Sigma_{n}$ and $\Pi_{n}$ formulas for $n \in \omega$. We refer to these latter classes as $\Sigma_{n}^{c}$ and $\Pi_{n}^{c}$ for brevity. We note that $\Sigma_{0}, \Pi_{0}, \Sigma_{0}^{c}$ and $\Pi_{0}^{c}$ all denote the class of finitary quantifier-free formulas of $L$. See [AK00, Chapters 6 and 7] for more on these formula classes. We will also occasionally use the nonstandard notation $\Sigma_{n}^{c, X}$ to mean the fragment of $\Sigma_{n}$ formulas where conjunctions and disjunctions are required to be $X$-c.e. So, $\Sigma_{n}^{c, 0}=\Sigma_{n}^{c}$.

In this paper we consider only countable Boolean algebras and use the signatures $\wedge, \vee,-, 0,1$, but otherwise follow the standard reference Mon89. We will also write $\dot{V}$ for a disjoint union. In what follows Boolean algebras are denoted by $\mathcal{A}, \mathcal{B}, \mathcal{C}$ and their elements by $a, b, c$. We write $\mathcal{A} \oplus \mathcal{B}$ for the direct sum of $\mathcal{A}$ and $\mathcal{B}$ and $\mathcal{A}\lceil a=\{b \in \mathcal{A}: b \leq a\}$ for the relative subalgebra of $\mathcal{A}$. We allow Boolean algebras to consist of one element; these are the trivial Boolean algebras. The class of all countable Boolean algebras is BA.

A partition of an element $a$ in a Boolean algebra $\mathcal{A}$ is a finite sequence $a_{0}, \ldots, a_{k}$ of pairwise disjoint elements (that is, $a_{i} \wedge a_{j}=0$ for all $i \neq j$ ) which join to $a$. A partition of a Boolean algebra $\mathcal{A}$ is a partition of its unit, $1_{\mathcal{A}}$. We will write $a=a_{0} \dot{\vee} \ldots \dot{\vee} a_{k}$ to mean that $a_{0}, \ldots, a_{k}$ is a partition of $a$. Note that if $1_{\mathcal{A}}=a_{0} \dot{\vee} \ldots \dot{\vee} a_{k}$, then $\mathcal{A}$ is isomorphic to the $\operatorname{disjoint} \operatorname{sum} \mathcal{A}\left\lceil a_{0} \oplus \ldots \oplus \mathcal{A}\left\lceil a_{k}\right.\right.$.

2.2. Back-and-forth relations. The main notion studied in $[\mathrm{HM}$ is that of $n$ back-and-forth relations of Boolean algebras. The purpose behind the use of these relations is to identify the computable Boolean algebras which cannot be distinguished by $0^{(n)}$.

Definition 2.1 ([AK00, $\S 15.3 .4])$. Let $\mathcal{A}$ and $\mathcal{B}$ be Boolean algebras. Let $\mathcal{A} \leq_{0} \mathcal{B}$ if either both $\mathcal{A}$ and $\mathcal{B}$ are trivial one-element Boolean algebras, or neither is. For $n>0, \mathcal{A} \leq_{n} \mathcal{B}$ if for every partition $1_{\mathcal{B}}=b_{0} \dot{\vee} \ldots \dot{\vee} b_{k}$ of $\mathcal{B}$, there is a partition $1_{\mathcal{A}}=a_{0} \dot{\vee} \ldots \dot{\vee} a_{k}$ of $\mathcal{A}$ such that $\mathcal{B}\left\lceil b_{i} \leq_{n-1} \mathcal{A}\left\lceil a_{i}\right.\right.$ for each $i \leq k$. Let $\mathcal{A} \equiv_{n} \mathcal{B}$ if $\mathcal{A} \leq_{n} \mathcal{B}$ and $\mathcal{B} \leq_{n} \mathcal{A}$

Theorem 2.2 (Karp; Ash and Knight). Let $\mathcal{A}$ and $\mathcal{B}$ be non-isomorphic Boolean algebras. The following are equivalent:

(1) Given a Boolean algebra $\mathcal{C}$ that is isomorphic to either $\mathcal{A}$ or $\mathcal{B}$, deciding whether $\mathcal{C} \cong \mathcal{A}$ is (boldface) $\boldsymbol{\Sigma}_{n}^{0}$-hard.

(2) All the infinitary $\Sigma_{n}$ sentences true in $\mathcal{B}$ are true in $\mathcal{A}$.

(3) $\mathcal{A} \leq_{n} \mathcal{B}$.

Sketch of the proof. The equivalence of (2) and (3) is due to Karp; see AK00, Proposition 15.1]. For (11) $\Rightarrow$ (2), note that if there is an infinitary $\Sigma_{n}$ sentence $\varphi$ that is true in $\mathcal{B}$ but not in $\mathcal{A}$, then to decide whether $\mathcal{C} \cong \mathcal{A}$, all we have to do is check whether $\mathcal{C} \models \varphi$, and if so, we know that $\mathcal{C} \cong \mathcal{B}$. Checking whether $\mathcal{C} \models \varphi$ holds is $\boldsymbol{\Sigma}_{n}^{0}$, so deciding whether $\mathcal{C} \cong \mathcal{A}$ is $\boldsymbol{\Pi}_{n}^{0}$, and hence not $\boldsymbol{\Sigma}_{n}^{0}$-hard. For (2) $\Rightarrow$ (11) we use [AK00, Theorem 18.6]. Let $Z$ be a set that can compute the $n$-back-and-forth relations among tuples of elements from $\mathcal{A}$ and $\mathcal{B}$. Relative to $Z$, we have that $\mathcal{A}$ and $\mathcal{B}$ are $n$-friendly. Let $\varphi(X)$ be a $\boldsymbol{\Sigma}_{n}^{0}$ formula of arithmetic with real parameters and a real free variable $X$, and assume that $Z$ was also chosen to 
compute all the real parameters in $\varphi(X)$. Using the uniformity in AK00, Theorem 18.6] we obtain a $Z$-computable procedure (hence a continuous function) that given $X$, produces a Boolean algebra $\mathcal{C}_{X}$ such that

$$
\mathcal{C}_{X} \cong \begin{cases}\mathcal{A} & \text { if } \varphi(X), \\ \mathcal{B} & \text { if } \neg \varphi(X) .\end{cases}
$$

(See [Kec95, §22B] for more on boldface hard classes.)

In $[\mathrm{HM}]$ we studied the family of ordered monoids

$$
\left(\mathbf{B A} / \equiv_{n}, \leq_{n}, \oplus\right) .
$$

We call the equivalence classes bf-types, or $n$-bf-types. We showed that there is a computably presentable ordered monoid $\left(\mathbf{I N V}_{n},+, \leq_{n}\right)$ with

$$
\left(\mathbf{B A} / \equiv_{n}, \oplus, \leq_{n}\right) \cong\left(\mathbf{I N V}_{n},+, \leq_{n}\right),
$$

and we built a map $T_{n}$ from Boolean algebras to $\mathbf{I N V}_{n}$ such that

$$
\mathcal{A} \leq_{n} \mathcal{B} \Longleftrightarrow T_{n}(\mathcal{A}) \leq_{n} T_{n}(\mathcal{B}) \quad \text { and } \quad T_{n}(\mathcal{A} \oplus \mathcal{B})=T_{n}(\mathcal{A})+T_{n}(\mathcal{B}) .
$$

These results are summarized in this section.

To our knowledge, the back-and-forth equivalence classes through the first five levels (i.e., $\equiv_{0}, \ldots, \equiv_{4}$ ) were first described by [la04. Here are the first four levels:

- $\mathcal{A} \leq_{0} \mathcal{B}$ if and only if $(|\mathcal{A}|=1 \Longleftrightarrow|\mathcal{B}|=1)$.

- $\mathcal{A} \leq_{1} \mathcal{B}$ if and only if $\mathcal{A} \leq_{0} \mathcal{B}$ and $|\mathcal{A}| \geq|\mathcal{B}|$.

- $\mathcal{A} \leq_{2} \mathcal{B}$ if and only if $|\mathcal{A}|=|\mathcal{B}|$ and $|\operatorname{At}(\mathcal{A})| \geq|\operatorname{At}(\mathcal{B})|$, where $\operatorname{At}(\mathcal{B})$ is the set of atoms of $\mathcal{B}$.

- $\mathcal{A} \leq_{3} \mathcal{B}$ if and only if $|\mathcal{A}|=|\mathcal{B}|,|\operatorname{At}(\mathcal{A})|=|\operatorname{At}(\mathcal{B})|,(\mathcal{A}$ atomic $\Rightarrow \mathcal{B}$ atomic $)$, and $|\mathcal{A} / F(\mathcal{A})| \geq|\mathcal{B} / F(\mathcal{B})|$, where $F(\mathcal{A})$ is the ideal of finite joins of atoms in $\mathcal{A}$.

The key to our investigation of the $n$-back-and-forth types in [HM are the $n$ indecomposable Boolean algebras:

Definition $2.3([\mathrm{HM}])$. A Boolean algebra $\mathcal{A}$ is $n$-indecomposable if for every partition $1_{\mathcal{A}}=a_{0} \dot{\vee} \ldots \dot{\vee} a_{k}$, there is some $i \leq k$ with $\mathcal{A} \equiv_{n} \mathcal{A}\left\lceil a_{i}\right.$.

Example 2.4. Let us see what the indecomposable algebras are in the first few levels of the back-and-forth hierarchy. The trivial algebra and two-element algebra are clearly $n$-indecomposable for every $n$. The infinite interval algebras $\operatorname{Int}(\omega)$ (one-atom) and $\operatorname{Int}(\eta)$ (atomless) are also $n$-indecomposable for every $n$, since for any partition of these algebras, there is always an isomorphic subalgebra.

- Every algebra is 0-indecomposable. There are two classes: the trivial algebras and everything else.

- There are three classes of 1-indecomposable algebras: the trivial algebras, the two-element algebras and the infinite algebras. These are the algebras that bound zero, one and infinitely many elements.

- There are four classes of 2-indecomposable algebras. Besides the trivial algebras and two-element algebras, there are two types of 2-indecomposable infinite algebras: those that bound zero atoms (atomless algebras) and those that bound infinitely many atoms. 
- There are six classes of 3-indecomposable algebras. Besides the trivial algebras, two element algebras and atomless algebras, there are three types of 3-indecomposable algebras with infinitely many atoms:

- atomic algebras $\mathcal{A}$ for which $|\mathcal{A} / F(\mathcal{A})|=1$ (all of which are isomorphic to the interval algebra $\operatorname{Int}(\omega))$;

- atomic algebras $\mathcal{A}$ for which $|\mathcal{A} / F(\mathcal{A})|$ is infinite (for example, $\operatorname{Int}\left(\omega^{2}\right)$ and $\operatorname{Int}(\omega \cdot \eta))$; and

- non-atomic algebras which bound infinitely many atoms and cannot be split into an atomic and an atomless part (for example, $\operatorname{Int}(\omega+\eta)$ and $\left.\operatorname{Int}\left(\omega^{2}+\eta\right)\right)$.

The Boolean algebra $\operatorname{Int}(\omega) \oplus \operatorname{Int}(\eta)$ is 2 -indecomposable, since it bounds infinitely many atoms (any partition of it must include a subalgebra also bounding infinitely many atoms), but it is not 3 -indecomposable. The reason is that $\operatorname{Int}(\omega) \oplus \operatorname{Int}(\eta)$ is non-atomic as well as bounding infinitely many atoms, but $\operatorname{Int}(\omega)$ is atomic and $\operatorname{Int}(\eta)$ bounds no atoms.

The main results we proved about the $n$-indecomposable Boolean algebras are summarized in the following theorem.

Theorem 2.5 ([M] $)$. For each $n$,

(a) There are only finitely many $n$-back-and-forth equivalence classes among the $n$-indecomposable algebras.

(b) Every Boolean algebra can be decomposed into a finite sum of n-indecomposable subalgebras.

(c) $\mathcal{A} \leq_{n+1} \mathcal{B}$ if and only if for every partition $1_{\mathcal{B}}=b_{0} \dot{\vee} \ldots \dot{\vee} b_{k}$ of $\mathcal{B}$ into $n$-indecomposable subalgebras, there is a partition $1_{\mathcal{A}}=a_{0} \dot{V} \ldots \dot{V} a_{k}$ of $\mathcal{A}$ such that $\mathcal{B}\left\lceil b_{i} \leq_{n} \mathcal{A}\left\lceil a_{i}\right.\right.$ for each $i \leq k$. (If $\mathcal{A}$ is $(n+1)$-indecomposable, then the partition of $\mathcal{A}$ can also be restricted to $n$-indecomposables, [HM, Lemma 4.3], although this is not generally true, [HM, Example 7.21].)

The theorem suggests that we may understand the $(n+1)$-back-and-forth relations between Boolean algebras by knowing how these algebras can be partitioned into $n$-indecomposable subalgebras.

2.3. Back-and-forth invariants for indecomposables. We start by defining what we call $n$-back-and-forth invariants on $n$-indecomposable elements of a Boolean algebra, where an element $a \in \mathcal{A}$ is $n$-indecomposable if $\mathcal{A}\lceil a$ is $n$-indecomposable. For each $n$, we define a finite set $\mathbf{B F}_{n}$ of combinatorial objects that we will use as back-and-forth invariants. Each $\mathbf{B} \mathbf{F}_{n}$ is a subset of the power set of $\mathbf{B} \mathbf{F}_{n-1}$, and $\mathbf{B F}_{0}=\{*\}$, where $*$ is just a symbol. On each $\mathbf{B F}_{n}$, we will define a partial ordering $\leq_{n}$ in a combinatorial way. (See Definition 2.7 below.) Before defining $\mathbf{B F}_{n}$ and $\leq_{n}$, we define the invariant maps.

Definition 2.6. For $X \subseteq \mathbf{B F}_{n}$ we let $\max X$ be the antichain of $\leq_{n}$-maximal elements of $X$. Let $\operatorname{dc} X \subseteq \mathbf{B F}_{n}$ be the $\leq_{n}$-downward closure of $X$. To each $n$-indecomposable element $a \neq 0_{\mathcal{A}}$ in a Boolean algebra $\mathcal{A}$ with more than one 
element we assign an element of $\mathbf{B F}_{n}$ as follows:

$$
\begin{aligned}
t_{0}(a)= & * . \\
\hat{t}_{n+1}(a)= & \left\{\alpha \in \mathbf{B F}_{n}:\right. \\
& \left.\forall k \exists a_{1}, \ldots, a_{k}<a, \text { all disjoint, } n \text {-indecomposable, with } t_{n}\left(a_{i}\right)=\alpha\right\} . \\
t_{n+1}(a)= & \max \hat{t}_{n+1}(a) .
\end{aligned}
$$

Let $t_{n}(\mathcal{A})=t_{n}\left(1_{\mathcal{A}}\right)$.

This definition is not the same as the one we gave in [HM, Definition 3.3], but it is equivalent, as it can be shown using [HM, Lemma 3.15]. (In [HM, Lemma 3.15] we use the notation $\alpha \in \in^{\mathrm{w}} \beta$ for $\alpha \in \mathrm{dc} \beta$. We also use the notion of $n$ indecomposable for an ultrafilter, which, by [HM, Theorem 4.1], is equivalent to our notion.) We note, just to help the intuition, that $t_{n+1}(a)$ can also be characterized as the antichain of $\mathbf{B} \mathbf{F}_{n}$ which satisfies the following condition: $\alpha \in \operatorname{dc} t_{n+1}(a)$ iff there exists an infinite sequence $a_{1}, a_{2}, \ldots$ of disjoint, $n$-indecomposable elements below $a$ such that for all $i, t_{n}\left(a_{i}\right) \geq_{n} \alpha$.

We define $\mathbf{B F}_{n}$ to be the set of subsets of $\mathbf{B F}_{n-1}$ which appear in the image of the map $t_{n}$ over all Boolean algebras. All the elements of $\mathbf{B F}_{n}$ are $\leq_{n}$-antichains from $\mathbf{B F}_{n-1}$, but not all antichains are in this image. In [HM, Section 5] we characterize this image in terms of a combinatorial property.

Notice that $\hat{t}_{n}(a)$ contains more information about $a$ than $t_{n}(a)$. The reason why we choose to work with $t_{n}(a)$ is that (as we will see) the information contained in $t_{n}(a)$ is exactly what can be decoded with $n$ Turing jumps, whereas the information in $\hat{t}_{n}(a)$ is more extensive than this.

We now define a relation $\leq_{n}$ on $\mathbf{B F}_{n}$ so that the following holds: for any $n$ indecomposable Boolean algebras $\mathcal{A}$ and $\mathcal{B}$,

$$
\mathcal{A} \leq_{n} \mathcal{B} \Longleftrightarrow t_{n}(\mathcal{A}) \leq_{n} t_{n}(\mathcal{B}) .
$$

We will also define a projection map $(\cdot)_{n}: \mathbf{B F}_{n+1} \rightarrow \mathbf{B F}_{n}$ such that for every $(n+1)$-indecomposable Boolean algebra $\mathcal{A}$,

$$
\left(t_{n+1}(\mathcal{A})\right)_{n}=t_{n}(\mathcal{A}) .
$$

These results and the following definition are from [HM, Section 3].

Definition 2.7. By induction on $n$, we define a relation $\leq_{n}$ on $\mathbf{B F}_{n}$ and a map $(\cdot)_{n}: \mathbf{B F}_{n+1} \rightarrow \mathbf{B F}_{n}$.

- On $\mathbf{B F}_{0}=\{*\}$, let $* \leq_{0} *$.

- For $\alpha \in \mathbf{B F}_{n+1}$, let $(\alpha)_{n}=\max \left\{(\gamma)_{n-1}: \gamma \in \alpha\right\} \in \mathbf{B F}_{n}$, unless $n=0$, in which case $(\alpha)_{0}=*$.

- For $\alpha, \beta \in \mathbf{B F}_{n+1}$, let $\alpha \leq_{n+1} \beta$ if $(\alpha)_{n} \equiv_{n}(\beta)_{n}$ and $\operatorname{dc} \beta \subseteq \operatorname{dc} \alpha$.

- For $\alpha, \beta \in \mathbf{B F}_{n+1}$, let $\alpha \equiv_{n+1} \beta$ if $\alpha \leq_{n+1} \beta$ and $\beta \leq_{n+1} \alpha$.

In [HM, Lemma 3.9] we showed that $\left(t_{n+1}(\mathcal{A})\right)_{n}=t_{n}(\mathcal{A})$ whenever $\mathcal{A}$ is $(n+1)$ indecomposable. It follows that $(\cdot)_{n}: \mathbf{B F}_{n+1} \rightarrow \mathbf{B F}_{n}$.

For each $\alpha \in \mathbf{B F}_{n}$, there is a computable $n$-indecomposable Boolean algebra $\mathcal{A}_{\alpha}$ with $t_{n}\left(\mathcal{A}_{\alpha}\right)=\alpha[\mathrm{HM}$, Section 5]. Using this representative algebra, define a predicate $\mathrm{R}_{\alpha}$ over each Boolean algebra $\mathcal{B}$ as follows: for each element $b \in \mathcal{B}$, let

$$
\mathcal{B}=\mathrm{R}_{\alpha}(b) \Longleftrightarrow \mathcal{B}\left\lceil b \geq_{n} \mathcal{A}_{\alpha} .\right.
$$


These predicates are $\Pi_{n}^{c}[\mathbf{H M}$, Lemma 8.9]. The next theorem shows that together the predicates $\mathbf{R}_{\alpha}$ for $\alpha \in \mathbf{B F}_{n}$ capture all the structural information of a Boolean algebra that can be obtained with $n$ Turing jumps.

Theorem 2.8. Let $\mathcal{B}$ be a Boolean algebra, $R \subseteq \mathcal{B}$ and $n \in \omega$. The following are equivalent:

(1) $R$ is relatively intrinsically $\Sigma_{n+1}$. That is, if $\mathcal{A} \cong \mathcal{B}$ and $(\mathcal{A}, Q) \cong(\mathcal{B}, R)$, then $Q$ is a $\Sigma_{n+1}^{0}(\mathcal{A})$ subset of $\omega$.

(2) $R$ is explicitly $\Sigma_{n+1}$. That is, $R$ can be defined in $\mathcal{B}$ by a computable infinitary $\Sigma_{n+1}^{c}$ formula with finitely many parameters from $\mathcal{B}$.

(3) There exists a $0^{(n)}$-computable sequence $\left\{\varphi_{i}: i \in \omega\right\}$ of finitary $\Sigma_{1}$ formulas that use the predicates $\mathrm{R}_{\alpha}$ for $\alpha \in \boldsymbol{B F}_{n}$, together with a finite tuple of parameters $\bar{p} \in \mathcal{B}$ such that

$$
x \in R \Longleftrightarrow \bigvee_{i \in \omega} \varphi_{i}(\bar{p}, x) .
$$

The equivalence of the first two statements is due to Ash, Knight, Manasse and Slaman, and Chisholm (see AK00, Theorem 10.1]). The equivalence of the latter two statements is proved in [HM, Theorem 8.11]. Furthermore, it was proved there that there is a uniformly computable way to go from a $\Sigma_{n+1}^{c}$ formula defining $R$ as in (2) to an index for a $0^{(n)}$-computable sequence of formulas as in (3).

The importance of the predicates $\left\{\mathrm{R}_{\alpha}: \alpha \in \mathbf{B F}_{n}\right\}$ leads to the following definition, stated previously in Definition 1.1.

Definition 2.9. An $n$-Boolean algebra ( $n$-algebra, for short), denoted by $\mathrm{B}_{n}(\mathcal{A})$, is a structure $\left(A, \vee, \wedge,-, 0,1, \mathrm{R}_{\alpha}: \alpha \in \mathbf{B F}_{n}\right)$ where $\mathcal{A}=(A, \vee, \wedge,-, 0,1)$ is a Boolean algebra. If $\mathrm{B}_{n}(\mathcal{A})$ is $Y$-computable, we say that $\mathcal{A}$ is $n$-Y-approximable.

Using the notation from Montalbán Mon, Theorem 2.8 states that $\mathrm{B}_{n}(\mathcal{A})$ is the nth jump of the structure $\mathcal{A}$. The next theorem, a corollary of Theorem 2.8 , provides alternative characterizations of $n$ - $0^{n}$-approximable algebras.

Theorem 2.10. Let $\mathcal{A}$ be a presentation of a Boolean algebra. The following are equivalent:

(a) $B_{n}(\mathcal{A})$ is computable in $0^{(n)}$.

(b) The $\Sigma_{n+1}^{c}$-diagram of $\mathcal{A}$ is a $\Sigma_{n+1}^{0}$ set of formulas.

(c) The $\Pi_{n}^{c}$-diagram of $\mathcal{A}$ is computable in $0^{(n)}$.

The following theorem is a consequence of [Mon, Theorem 3.1].

Theorem 2.11. Let $\mathcal{A}$ be a Boolean algebra, and let $Z$ be any set. If $B_{n}(\mathcal{A})$ is $Z^{(n)}$-computable, then $\mathcal{A}$ has a $Z$-lown copy. Furthermore, an isomorphism between $\mathcal{A}$ and its $Z$-lown copy can be computed by $Z^{(n)}$.

In our main construction, we will build a $5-0^{(5)}$-approximable Boolean $\mathcal{A}$ algebra which is not $0^{(7)}$-isomorphic to any computable Boolean algebra. Then using Theorem 2.11, we will get that $\mathcal{A}$ is $0^{(5)}$-isomorphic to a low 5 Boolean algebra. This low $_{5}$ Boolean algebra is then also not $0^{(7)}$-isomorphic to any computable one.

2.4. General back-and-forth invariants. Let $\left(\overline{\mathbf{I N V}}_{n},+, 0\right)$ be the free commutative monoid with generators $\mathbf{B F}_{n}$. In [HM, Definition 7.7] we give a purely combinatorial definition of the relation $\leq_{n}$ on $\overline{\mathbf{I N V}}_{n}$. (This definition is based on 
the notion of a partition of an element of $\overline{\mathbf{I N V}}_{n}$ which is a bit cumbersome, so we will not include it here; see [HM, Definition 7.6] for details.) The main property of this definition is that, if we have $\alpha_{1}, \ldots, \alpha_{k}, \beta_{1}, \ldots, \beta_{\ell} \in \mathbf{B F}_{n}$, and we have $n$-indecomposable Boolean algebras $\mathcal{A}_{\gamma}$ of bf-type $\gamma$, then

$$
\mathcal{A}_{\alpha_{1}} \oplus \cdots \oplus \mathcal{A}_{\alpha_{k}} \leq_{n} \mathcal{A}_{\beta_{1}} \oplus \cdots \oplus \mathcal{A}_{\beta_{\ell}} \Longleftrightarrow \alpha_{1}+\cdots+\alpha_{k} \leq_{n} \beta_{1}+\cdots+\beta_{k} .
$$

Recall that for each $\alpha \in \mathbf{B F}_{n}$ there is an $n$-indecomposable Boolean algebra $\mathcal{A}_{\alpha}$ with bf-type $\alpha$, so that for each $\sigma=\alpha_{1}+\cdots+\alpha_{k} \in \overline{\mathbf{I N V}}_{n}$, there is a Boolean algebra $\mathcal{A}_{\sigma}=\mathcal{A}_{\alpha_{1}} \oplus \cdots \oplus \mathcal{A}_{\alpha_{k}}$. Thus, this ordering on $\overline{\mathbf{I N V}}_{n}$ induces an equivalence relation $\equiv_{n}$ on $\overline{\mathbf{I N V}}_{n}$; let $\mathbf{I N V} \mathbf{V}_{n}$ be the quotient structure $\left(\mathbf{I N V} \mathbf{V}_{n},+, 0, \leq_{n}\right)$, which is a commutative ordered monoid by [HM, Theorem 7.10]. The invariant map from Boolean algebras to $\mathbf{I N V} \mathbf{V}_{n}$ is now defined in the obvious way:

Definition 2.12. Given a Boolean algebra $\mathcal{B}$, let $\mathcal{B}_{0} \oplus \cdots \oplus \mathcal{B}_{k}$ be a partition of $\mathcal{B}$ into $n$-indecomposable Boolean algebras. Let $T_{n}(\mathcal{B}) \in \mathbf{I N V}_{n}$ be the $\equiv_{n}$-equivalence class of

$$
t_{n}\left(\mathcal{B}_{0}\right)+\ldots+t_{n}\left(\mathcal{B}_{k}\right) \in \overline{\mathbf{I N V}}_{n}
$$

We proved in $[\mathrm{HM}$, Section 7.4] that this definition is independent of the choice of the partition $\mathcal{B}_{0} \oplus \cdots \oplus \mathcal{B}_{k}$ of $\mathcal{B}$ and that

$$
T_{n}:\left(\mathbf{B A} / \equiv_{n}, \oplus, \leq_{n}\right) \rightarrow\left(\mathbf{I N V}_{n},+, \leq_{n}\right)
$$

is an isomorphism. We also extend the projection map to general invariants, $(\cdot)_{n}$ : $\mathbf{I N V}_{n+1} \rightarrow \mathbf{I N V}_{n}$, by

$$
\left(\alpha_{0}+\ldots+\alpha_{k}\right)_{n}=\left(\alpha_{0}\right)_{n}+\ldots+\left(\alpha_{k}\right)_{n} .
$$

The projection map also satisfies the property that

$$
\left(T_{n+1}(\mathcal{A})\right)_{n}=T_{n}(\mathcal{A})
$$

for every Boolean algebra $\mathcal{A}$.

Remark 2.13. The relation $\leq_{n}$ on $\overline{\mathbf{I N V}}_{n}$ agrees with $\leq_{n}$ on $\mathbf{B} \mathbf{F}_{n}$, when restricted to the $n$-indecomposable bf-types (see [HM, Section 7.3]). For the remainder of the paper, we will be working with general invariants, $\mathbf{I N V} \mathbf{V}_{n}$, which are $\equiv_{n}$-equivalence classes of $\overline{\mathbf{I N V}}_{n}$. Thus, we will abuse notation and think of $\mathbf{B F}_{n}$ as a subset of INV $_{n}$.

Remark 2.14. One consequence of the definition of $\leq_{n}$ is that for $\alpha_{0}, \ldots, \alpha_{k}, \beta_{0}, \ldots$, $\beta_{\ell} \in \mathbf{B F}_{n}$, if

$$
\alpha_{0}+\ldots+\alpha_{k} \leq_{n} \beta_{0}+\ldots+\beta_{\ell}
$$

then

$$
\mathrm{dc} \bigcup_{j \leq \ell} \beta_{j} \subseteq \mathrm{dc} \bigcup_{i \leq k} \alpha_{i}
$$

(see [HM, Theorem 7.18]). Thus, if

$$
\alpha_{0}+\ldots+\alpha_{k} \equiv_{n} \beta_{0}+\ldots+\beta_{\ell},
$$

then

$$
\operatorname{dc} \bigcup_{j \leq \ell} \beta_{j}=\mathrm{dc} \bigcup_{i \leq k} \alpha_{i} \quad \text { and } \quad \max \bigcup_{j \leq \ell} \beta_{j}=\max \bigcup_{i \leq k} \alpha_{i}
$$

So, for $\sigma=\alpha_{0}+\ldots+\alpha_{k} \in \overline{\mathbf{I N V}}_{n}$, we write $\operatorname{dc} \sigma$ for $\operatorname{dc} \bigcup_{i \leq k} \alpha_{i}$ and $\max \sigma$ for $\max \bigcup_{i \leq k} \alpha_{i}$, and we have that $\operatorname{dc} \sigma$ and $\max \sigma$ are independent of the representative $\sigma$ of its equivalence class in $\mathbf{I N V}_{n}$. 
We note, just to help the intuition, that $\operatorname{dc} T_{n+1}(\mathcal{A})$ can be characterized as the subset of $\mathbf{B F}_{n}$ which satisfies the following condition: $\alpha \in \operatorname{dc} T_{n+1}(\mathcal{A})$ iff there exists an infinite sequence $a_{1}, a_{2}, \ldots$ of disjoint, $n$-indecomposable elements such that for all $i, t_{n}\left(a_{i}\right) \geq_{n} \alpha$.

Another consequence of the definition of $\leq_{n}$ on $\overline{\mathbf{I N V}}_{n}$ is the following theorem, which is nearly identical to [HM, Theorem 7.18]. (The only essential change is to replace the more restrictive simple partitions of invariants in the statement of [HM, Theorem 7.18] with the general partitions found here.)

Theorem 2.15. Let $\sigma, \tau \in \boldsymbol{I N} \boldsymbol{V}_{n}$. The following are equivalent:

(1) $\sigma \leq_{n} \tau$.

(2) For each $\tau_{0}, \ldots, \tau_{k} \in \boldsymbol{I} \boldsymbol{N} \boldsymbol{V}_{n}$ with $\tau=\tau_{0}+\ldots+\tau_{k}$, there are $\sigma_{0}, \ldots, \sigma_{k} \in$ $\boldsymbol{I N} \boldsymbol{V}_{n}$ with $\sigma=\sigma_{0}+\ldots+\sigma_{k}$ such that $\left(\tau_{i}\right)_{n-1} \leq_{n-1}\left(\sigma_{i}\right)_{n-1}$ for each $i \leq k$.

(3) Both of the following hold:

(a) $\operatorname{dc} \tau \subseteq \operatorname{dc} \sigma$.

(b) There exists some $\beta_{0}, \ldots, \beta_{k} \in \boldsymbol{B F}_{n}$ with $\tau=\beta_{0}+\ldots+\beta_{k}$ and $\sigma_{0}, \ldots, \sigma_{k} \in \boldsymbol{I N} \boldsymbol{V}_{n}$ with $\sigma=\sigma_{0}+\ldots+\sigma_{k}$ such that $\left(\beta_{i}\right)_{n-1} \leq_{n-1}$ $\left(\sigma_{i}\right)_{n-1}$ for each $i \leq k$.

Example 2.16. It is possible for a Boolean algebra to have an $n$-indecomposable bf-type from $\mathbf{I N V}_{n}$ without being itself $n$-indecomposable. For example, let $\mathcal{A}=$ $\operatorname{Int}(\omega+\eta)$ and $\mathcal{B}=\operatorname{Int}(\omega) \oplus \operatorname{Int}(\eta)$. Using the conditions for $\leq_{3}$ in Section 2.2. we have $\mathcal{A} \equiv_{3} \mathcal{B}$; however, $\mathcal{B}$ is not 3 -indecomposable because $\mathcal{B} \not_{3} \operatorname{Int}(\omega)$ (the second algebra is atomic, but $\mathcal{B}$ is not) and $\mathcal{B} \neq_{3} \operatorname{Int}(\eta)$ (the second algebra has no atoms, but $\mathcal{B}$ has infinitely many atoms).

We will usually be considering a single Boolean algebra $\mathcal{A}$ and will be interested in the bf-types of relative subalgebras $\mathcal{A}\left\lceil a\right.$ for $a \in \mathcal{A}$. We will write $T_{n}^{\mathcal{A}}(a)$ for $T_{n}(\mathcal{A}\lceil a)$.

\subsection{Exclusive bf-types.}

Definition 2.17. We say that $\alpha \in \mathbf{B F}_{n}$ is exclusive if $(\alpha)_{n-1} \notin \mathrm{dc} \alpha$.

In terms of Boolean algebras, if $\mathcal{A}$ is $n$-indecomposable and has an exclusive $n$-indecomposable type, then for every partition $a_{0}, \ldots, a_{\ell}$ of $\mathcal{A}$ there is a unique $i$ such that $\mathcal{A} \equiv_{n-1} \mathcal{A}\left\lceil a_{i}\right.$. On the other hand, if $\mathcal{A}$ is $n$-indecomposable but its $n$ bf-type is not exclusive, then there are infinitely many disjoint, $n$-indecomposable elements $a_{0}, a_{1}, \cdots \in \mathcal{A}$ with $t_{n-1}\left(a_{i}\right) \geq_{n-1} t_{n-1}(\mathcal{A})$. For example, the 1 -atom $\operatorname{Int}(\omega)$ is $n$-indecomposable for every $n$, but it has an exclusive $n$-indecomposable type only when $n \geq 3$. For $n=2$, $\operatorname{Int}(\omega)$ has the 2-indecomposable type $\{\{\}\}$, corresponding to an algebra bounding infinitely many atoms, as does the algebra $\operatorname{Int}(2 \cdot \eta)$ which does not have exclusive type for any $n$.

Up to level four, every exclusive $n$-indecomposable type is a $\leq_{n}$-maximal element in $\mathbf{B F}_{n}$. This is not the case at level five, and we will exploit this in our construction. In [HM, Definition 5.2] we introduced the relation $\triangleleft_{n}$ on $\mathbf{B F}_{n}$ :

Definition 2.18. For $\alpha, \beta \in \mathbf{B F}_{n}, \alpha \triangleleft_{n} \beta$ if $\beta \subseteq \operatorname{dc} \alpha$ and $(\beta)_{n-1} \in \operatorname{dc} \alpha$.

The significance of this relation is provided by the next two lemmas. 
Lemma 2.19 ([M, Lemma 7.12]). Let $\alpha, \beta_{0}, \ldots, \beta_{k} \in \boldsymbol{B F}_{n}$. The following are equivalent:

(1) $\alpha \leq_{n} \sum_{i \leq k} \beta_{i}$

(2) For some $i_{0} \leq k$ we have $\alpha \leq_{n} \beta_{i_{0}}$, and for every other $i \neq i_{0}$ we have $\alpha \triangleleft_{n} \beta_{i}$.

Lemma 2.20. The following holds for all $\alpha, \beta \in \boldsymbol{B F}_{n}$ :

$$
\alpha \triangleleft_{n} \beta \Longleftrightarrow \alpha \equiv_{n} \alpha+\beta \text {. }
$$

This last lemma is a consequence of [HM, Lemma 7.12] and [HM, Theorem 7.18(3)]. Note that $\alpha \in \mathbf{B F}_{n}$ is exclusive if and only if $\alpha \oiint_{n} \alpha$, so that $\alpha \neq_{n} \alpha+\alpha$. On the other hand, $\alpha$ is non-exclusive if and only if $\alpha \triangleleft_{n} \alpha$, so that $\alpha \equiv_{n} \alpha+\alpha$. This is the key fact about exclusivity that our construction exploits.

2.6. Partitions. The back-and-forth invariant for an algebra provides information on how the algebra can be partitioned into subalgebras. In this section we explore some of these implications using Lemma 2.19. The following distills the essence of what it means for an algebra to have an indecomposable type.

Lemma 2.21. Let $\mathcal{A}$ be a Boolean algebra, $\alpha \in \boldsymbol{B F}_{n}$ and $T_{n}(\mathcal{A})=\alpha$. Then for any $\delta_{1}, \ldots, \delta_{k} \in \alpha$, there is a partition $1_{\mathcal{A}}=a_{0} \dot{\vee} a_{1} \dot{\vee} \ldots \dot{\vee} a_{k}$ such that $T_{n-1}^{\mathcal{A}}\left(a_{i}\right)=\delta_{i}$ for each $i>0$.

Proof. Recall that for each $\alpha \in \mathbf{B F}_{n}$, there is an $n$-indecomposable algebra $\mathcal{A}_{\alpha}$ with $T_{n}\left(\mathcal{A}_{\alpha}\right)=\alpha$. We show that for each $\delta \in \alpha$, there is a $\delta^{*} \in \mathbf{B F}_{n}$ with both $\left(\delta^{*}\right)_{n-1}=\delta$ and $\alpha \triangleleft_{n} \delta^{*}$. By Definition 2.6 there is a partition $1_{\mathcal{A}_{\alpha}}=a_{0} \dot{\vee} a_{1}$ where $a_{1}$ is $(n-1)$-indecomposable and $T_{n-1}^{\mathcal{A}_{\alpha}}\left(a_{1}\right)=\delta$. Applying Theorem 2.5 (b) we can further partition $a_{1}=b_{0} \dot{\vee} \ldots \dot{\vee} b_{k}$ into $n$-indecomposables and let $\beta_{i}=T_{n}^{\mathcal{A}_{\alpha}}\left(b_{i}\right)$ for each $i \leq k$. Since $a_{1}$ is $(n-1)$-indecomposable there is some $i \leq k$ with $T_{n-1}^{\mathcal{A}_{\alpha}}\left(b_{i}\right)=\delta$; for concreteness let $i=0$. We can write $\alpha=\sigma+\beta_{0}+\cdots+\beta_{k}$ where $\sigma=T_{n}^{\mathcal{A}_{\alpha}}\left(a_{0}\right)$, so that by Lemma 2.19, either $\alpha \leq_{n} \beta_{0}$ or $\alpha \triangleleft_{n} \beta_{0}$. If the first case holds, then $\alpha \triangleleft_{n} \beta_{0}$ follows from $\left(\beta_{0}\right)_{n-1}=\delta \in \alpha$; thus in either case if we let $\delta^{*}=\beta_{0}$, then $\alpha \triangleleft_{n} \delta^{*}$.

We need one additional technical fact: there can be no $\delta \in \alpha$ with $\delta<_{n-1}(\alpha)_{n-1}$. If $\delta<_{n-1}(\alpha)_{n-1}$, then by the definition of $\leq_{n-1}$, $\operatorname{dc} \delta \not \subset \mathrm{dc}(\alpha)_{n-1}$. Suppose $\delta \in \alpha$. Then by the previous paragraph there is a $\delta^{*} \in \mathbf{B F}_{n}$ with $\alpha \triangleleft_{n} \delta^{*}$ and $\left(\delta^{*}\right)_{n-1}=\delta$, and so $\operatorname{dc} \delta^{*} \subset \operatorname{dc} \alpha$ which implies, by the definition of the operator ()$_{n-1}$, that $\operatorname{dc} \delta \subset \operatorname{dc}(\alpha)_{n-1}$. Thus, it is impossible that both $\delta<_{n-1}(\alpha)_{n-1}$ and $\delta \in \alpha$.

Now let $\mathcal{A}$ be a Boolean algebra, $\alpha \in \mathbf{B F}_{n}, T_{n}(\mathcal{A})=\alpha$ and any $\delta_{1}, \ldots, \delta_{k} \in \alpha$. For each $i \leq k$ let $\delta_{i}^{*} \in \mathbf{B F}_{n}$ with $\left(\delta_{i}^{*}\right)_{n-1}=\delta_{i}$ and $\alpha \triangleleft_{n} \delta_{i}^{*}$, so that $\alpha=\alpha+\delta_{1}^{*}+$ $\cdots+\delta_{k}^{*}$ by Lemma 2.19 . Let $\mathcal{B}=\mathcal{A}_{\alpha} \oplus \mathcal{A}_{\delta_{1}^{*}} \oplus \cdots \oplus \mathcal{A}_{\delta_{k}^{*}}$; thus $T_{n}(\mathcal{B})=\alpha$ and, moreover, there is a partition $1_{\mathcal{B}}=b_{0} \dot{V} \ldots \dot{V} b_{k}$ into $n$-indecomposable elements with $T_{n-1}^{\mathcal{B}}\left(b_{i}\right)=\delta_{i}$ for each $i \geq 1$. Since $\mathcal{B} \equiv_{n} \mathcal{A}$, there is a matching partition $1_{\mathcal{A}}=c_{0} \dot{\vee} \ldots \dot{\vee} c_{k}$ with $b_{i} \leq_{n-1} c_{i}$ for each $i \leq k$. For each $i \geq 1$ we can further refine $c_{i}=d_{0} \dot{V} \ldots \dot{V} d_{\ell}$ so that each $d_{j}$ is $n$-indecomposable, and since $\delta_{i} \leq_{n-1} T_{n-1}^{\mathcal{A}}\left(d_{0}\right)+\cdots+T_{n-1}^{\mathcal{A}}\left(d_{\ell}\right)$, there is some $j \leq \ell$ with $\delta_{i} \leq_{n-1} T_{n-1}^{\mathcal{A}}\left(d_{j}\right)$. In fact, we must have equality: we can write $\alpha=\sigma+T_{n}^{\mathcal{A}}\left(d_{j}\right)$, where $\sigma=T_{n}^{\mathcal{A}}\left(1_{\mathcal{A}}-d_{j}\right)$, and so either $\alpha \leq_{n} T_{n}^{\mathcal{A}}\left(d_{j}\right)$ or $\alpha \triangleleft_{n} T_{n}^{\mathcal{A}}\left(d_{j}\right)$. In the first case we have $(\alpha)_{n-1}=$ $T_{n-1}^{\mathcal{A}}\left(d_{j}\right) \geq_{n-1} \delta_{i}$, so that $\delta_{i}=(\alpha)_{n-1}$ by the previous paragraph. In the second case we must also have $T_{n-1}^{\mathcal{A}}\left(d_{j}\right)=\delta_{i}$ by the maximality of $\delta_{i}$ in $\alpha$ and the fact that 
$T_{n-1}^{\mathcal{A}}\left(d_{j}\right) \in \operatorname{dc} \alpha$. Let $a_{i}=d_{j}$, so that $a_{i}$ is $n$-indecomposable and $T_{n-1}^{\mathcal{A}}\left(a_{i}\right)=\delta_{i}$. Doing this for each $i \geq 1$, we arrive at a partition $1_{\mathcal{A}}=a_{0} \dot{\vee} a_{1} \dot{V} \ldots \dot{V} a_{k}$ for which $T_{n-1}^{\mathcal{A}}\left(a_{i}\right)=\delta_{i}($ for $i \geq 1)$.

The following is a simple but useful lemma.

Lemma 2.22. If $\alpha, \gamma \in \boldsymbol{B F}_{n}, \sigma \in \boldsymbol{I N} \boldsymbol{V}_{n}$ and $\alpha=\alpha+\gamma+\sigma$, then $\alpha \triangleleft_{n} \gamma$.

Proof. Suppose $\alpha=\alpha+\gamma+\sigma$; then either $\alpha \triangleleft_{n} \gamma$ or both $\alpha \leq_{n} \gamma$ and $\alpha \triangleleft_{n} \alpha$. In the first case we are done. In the latter case, we have $(\alpha)_{n}=(\gamma)_{n}$ and $(\alpha)_{n} \in \alpha$, but since $\alpha \leq_{n} \gamma$ implies $\operatorname{dc} \gamma \subseteq \operatorname{dc} \alpha$, it follows that $\alpha \triangleleft_{n} \gamma$.

Lemma 2.21 applied generally to algebras with $n$-indecomposable bf-type; the next lemma refines this to the case where the algebra is itself $(n-1)$-indecomposable or $n$-indecomposable.

Lemma 2.23. Let $\mathcal{A}$ be an $(n-1)$-indecomposable Boolean algebra, $\alpha \in \boldsymbol{B F}_{n}$, $T_{n}(\mathcal{A})=\alpha$ and $\delta_{1}, \ldots, \delta_{k} \in \alpha$. Then there is a partition $1_{\mathcal{A}}=a_{0} \dot{\vee} a_{1} \dot{\vee} \ldots \dot{\vee} a_{k}$ such that $T_{n-1}^{\mathcal{A}}\left(a_{0}\right)=(\alpha)_{n-1}$ and $T_{n-1}^{\mathcal{A}}\left(a_{i}\right)=\delta_{i}$ for $i>0$. If, in addition, $\mathcal{A}$ is $n$-indecomposable, then we can get $T_{n}^{\mathcal{A}}\left(a_{0}\right)=\alpha$.

Proof. Let $\mathcal{A}$ be an $(n-1)$-indecomposable Boolean algebra, $\alpha \in \mathbf{B F}_{n}, T_{n}(\mathcal{A})=\alpha$ and $\delta_{1}, \ldots, \delta_{k} \in \alpha$. The construction of the required partition depends on whether $(\alpha)_{n-1} \in \alpha$. First, suppose $(\alpha)_{n-1} \notin \alpha$. By Lemma 2.21 there is a partition $1_{\mathcal{A}}=a_{0} \dot{\vee} a_{1} \dot{\vee} \ldots \dot{\vee} a_{k}$ such that $T_{n-1}^{\mathcal{A}}\left(a_{i}\right)=\delta_{i}$ for each $i>0$. Since $\mathcal{A}$ is $(n-1)$-indecomposable there must be some $i \leq k$ with $\mathcal{A} \equiv_{n-1} \mathcal{A}\left\lceil a_{i}\right.$, but since $(\alpha)_{n-1} \notin \alpha$, this must be $i=0$. If $\mathcal{A}$ is $n$-indecomposable, then $\mathcal{A} \equiv_{n} \mathcal{A}\left\lceil a_{0}\right.$. Next, suppose that $(\alpha)_{n-1} \in \alpha$ and set $\delta_{k+1}=(\alpha)_{n-1}$. Apply Lemma 2.21 to get a partition $1_{\mathcal{A}}=a_{0}^{\prime} \dot{\vee} a_{1} \dot{V} \ldots \dot{\vee} a_{k+1}$ with $T_{n-1}^{\mathcal{A}}\left(a_{i}\right)=\delta_{i}$ for each $i \geq 1$. Let $T_{n-1}^{\mathcal{A}}\left(a_{0}^{\prime}\right)=\beta_{0}+\cdots+\beta_{\ell}$. Then $(\alpha)_{n-1}=\beta_{0}+\cdots+\beta_{\ell}+\sigma+(\alpha)_{n-1}$ for some $\sigma$, so that by Lemma 2.22, $(\alpha)_{n-1} \triangleleft_{n-1} \beta_{j}$ for each $j \leq \ell$, and thus $T_{n-1}^{\mathcal{A}}\left(a_{0}^{\prime} \dot{\vee} a_{k+1}\right)=$ $(\alpha)_{n-1}$ by several applications of Lemma 2.20. Let $a_{0}=a_{0}^{\prime} \dot{\vee} a_{k+1}$. If $\mathcal{A}$ is $n$-indecomposable, then $\mathcal{A} \equiv_{n} \mathcal{A}\left\lceil\left(a_{0}^{\prime} \dot{\vee} a_{k+1}\right)\right.$ or $\mathcal{A} \equiv_{n} \mathcal{A}\left\lceil a_{i}\right.$ for some $i \geq 1$. In the first case, let $a_{0}=a_{0}^{\prime} \dot{\vee} a_{k+1}$. In the second case, $\delta_{i}=(\alpha)_{n-1}$, so let $a_{0}=a_{0}^{\prime} \dot{\vee} a_{i}$ and set $a_{i}=a_{k+1}$. It remains to show that $T_{n}^{\mathcal{A}}\left(a_{0}\right)=\alpha$. We can write $\alpha=\beta_{0}^{*} \dot{\vee} \ldots \dot{\vee} \beta_{\ell}^{*}+\alpha+\sigma$ for some $\sigma \in \mathbf{I N V}_{n}$, where $T_{n}^{\mathcal{A}}\left(a_{0}^{\prime}\right)=\beta_{0}^{*} \dot{\vee} \ldots \dot{\vee} \beta_{\ell}^{*}$. Then $\alpha \triangleleft_{n} \beta_{j}^{*}$ for each $j \geq 0$ by Lemma 2.22, and thus, $T_{n}^{\mathcal{A}}\left(a_{0}^{\prime} \dot{\vee} a_{i}\right)=\alpha$ by several applications of Lemma 2.20 .

As an application of Lemma 2.23 we prove a refinement of Theorem 2.5(C):

Lemma 2.24. Let $\mathcal{A}$ be an $n$-indecomposable Boolean algebra. Then for any algebra $\mathcal{B}, \mathcal{A} \leq_{n} \mathcal{B}$ if and only if for every partition $1_{\mathcal{B}}=b_{0} \dot{V} \ldots \dot{\vee} b_{k}$ with $T_{n-1}^{\mathcal{B}}\left(b_{i}\right) \in \boldsymbol{B F}_{n-1}$ for each $i \leq k$, there is a partition $1_{\mathcal{A}}=a_{0} \dot{V} \ldots \dot{\vee} a_{k}$ such that $T_{n-1}^{\mathcal{B}}\left(b_{i}\right) \leq_{n-1} T_{n-1}^{\mathcal{A}}\left(a_{i}\right)$ for each $i \leq k, T_{n}^{\mathcal{A}}\left(a_{j}\right)=T_{n}(\mathcal{A})$ for some $j$, and $T_{n-1}^{\mathcal{A}}\left(a_{i}\right) \in T_{n}(\mathcal{A})$ for all $i \neq j$.

Proof. The direction $(\Leftarrow)$ follows from Theorem 2.5 (c) . Conversely, suppose $\mathcal{A} \leq_{n}$ $\mathcal{B}$ and let $1_{\mathcal{B}}=b_{0} \dot{\vee} \ldots \dot{\vee} b_{k}$, where $T_{n-1}^{\mathcal{B}}\left(b_{i}\right) \in \mathbf{B F}_{n-1}$ for each $i \leq k$. Since $\mathcal{A} \leq_{n} \mathcal{B}$, there is a partition $1_{\mathcal{A}}=c_{0} \dot{\vee} \ldots \dot{\vee} c_{k}$ with $T_{n}^{\mathcal{A}}\left(c_{0}\right)=T_{n}(\mathcal{A})$ and $T_{n-1}^{\mathcal{B}}\left(b_{i}\right) \leq_{n-1} T_{n-1}^{\mathcal{A}}\left(c_{i}\right)$ for each $i \leq k$. We show that $T_{n-1}^{\mathcal{B}}\left(b_{i}\right) \in \operatorname{dc} T_{n}(\mathcal{A})$ for each 
$i>0$. Let $T_{n}^{\mathcal{A}}\left(c_{i}\right)=\gamma_{0}^{*}+\cdots+\gamma_{\ell}^{*}$. Then we can write $\alpha=\alpha+\gamma_{0}^{*}+\cdots+\gamma_{\ell}^{*}+\sigma$ where $T_{n}^{\mathcal{A}}\left(1_{\mathcal{A}}-\left(c_{0} \vee c_{i}\right)\right)=\sigma$, and thus $\alpha \triangleleft_{n} \gamma_{j}^{*}$ for each $j \leq \ell$. Let $\gamma_{j}=\left(\gamma_{j}^{*}\right)_{n-1}$, so that $\gamma_{j} \in \operatorname{dc} \alpha$ and $T_{n-1}^{\mathcal{A}}\left(c_{i}\right)=\gamma_{0}+\cdots+\gamma_{\ell}$. Now, $T_{n-1}^{\mathcal{B}}\left(b_{i}\right) \leq_{n-1} \gamma_{0}+\cdots+\gamma_{\ell}$, so there is some $j \leq \ell$ with $T_{n-1}^{\mathcal{B}}\left(b_{i}\right) \leq_{n-1} \gamma_{j}$ by Lemma 2.19, so let $\delta_{i} \in T_{n}(\mathcal{A})$ with $\delta_{i} \geq_{n-1} \gamma_{j}$. Thus, there are $\delta_{1}, \ldots, \delta_{k} \in T_{n}(\mathcal{A})$ with $T_{n-1}^{\mathcal{B}}\left(b_{i}\right) \leq_{n-1} \delta_{i}$ for each $i>0$. By Lemma 2.23, there is a partition $1_{\mathcal{A}}=a_{0} \dot{V} \ldots \dot{\vee} a_{k}$ with $T_{n}^{\mathcal{A}}\left(a_{0}\right)=T_{n}(\mathcal{A})$ and $T_{n-1}^{\mathcal{A}}\left(a_{i}\right)=\delta_{i}$ for each $i>0$.

\section{Boolean ALGEBra APPROXimations}

In this section we introduce the notion of an $n$-approximation of a Boolean algebra. This notion, which is one of the main applications of the work in [HM], is new, although it has roots in the work of others. The idea of using back-andforth increasing sequences of finite approximations appears in the work of Ash on $\alpha$-systems, but in a very general setting. (See Chapter 12 of AK00, on $n$-systems, and especially Section 15.6, for a motivation of these constructions, which bear a close relationship to our $n$-approximations.) In [KS00 they used finite Boolean algebras with additional predicates to approximate $\operatorname{low}_{n}$ Boolean algebras in their proof, for $n=1,2,3,4$, that every low $n$ Boolean algebra has a computable copy. Their construction in the low $_{4}$ case corresponds to what we call a 3-approximation, although we use the predicates $R_{\alpha}$ for $\alpha \in \mathbf{B F}_{3}$, while they used a finite set of predicates sufficient for defining these relations via Boolean combinations.

3.1. Finite labeled Boolean algebras. To approximate an $n$-Boolean algebra, we use finite $n$-labeled Boolean algebras.

Definition 3.1. A finite $n$-labeled Boolean algebra is a pair $\left(\mathcal{C}, \mathfrak{t}_{n}^{\mathcal{C}}\right)$, where $\mathcal{C}$ is a finite Boolean algebra and $\mathfrak{t}_{n}^{\mathcal{C}}: \mathcal{C} \rightarrow \mathbf{I N V}_{n}$ is such that

(1) every minimal element has an indecomposable $n$-bf-type;

(2) if $b=\bigvee_{i=0}^{k} a_{i}$, where each $a_{i}$ is a minimal element in $\mathcal{C}$, then $\mathfrak{t}_{n}^{\mathcal{C}}(b)=$ $\sum_{i=0}^{k} \mathfrak{t}_{n}^{\mathcal{C}}\left(a_{i}\right)$.

The minimal elements of a finite Boolean algebra are its atoms. We want to avoid using the word "atom" in this context, however, because we already use it to refer to one of the $n$-bf-types. A minimal element of $\mathcal{C}$ may or may not be labeled with the $n$-bf-type "atom".

Given two finite $n$-labeled Boolean algebras $\mathcal{C}_{0}$ and $\mathcal{C}_{1}$, we write

$$
\mathcal{C}_{0} \subseteq{ }_{n} \mathcal{C}_{1}
$$

if $\mathcal{C}_{0}$ is a subalgebra of $\mathcal{C}_{1}$ and for every $x \in \mathcal{C}_{0}, \mathfrak{t}_{n}^{\mathcal{C}_{0}}(x)=\mathfrak{t}_{n}^{\mathcal{C}_{1}}(x)$.

We write

$$
\mathcal{C}_{0} \leq{ }_{n} \mathcal{C}_{1}
$$

if $\mathcal{C}_{0}$ is a subalgebra of $\mathcal{C}_{1}$ and for every $x \in \mathcal{C}_{0}, \mathfrak{t}_{n}^{\mathcal{C}_{0}}(x) \leq_{n} \mathfrak{t}_{n}^{\mathcal{C}_{1}}(x)$.

Every $n$-labeled Boolean algebra is also an $(n-1)$-labeled Boolean algebra using the labeling function $\mathfrak{t}_{n-1}^{\mathcal{C}}(b)=\left(\mathfrak{t}_{n}^{\mathcal{C}}(b)\right)_{n-1}$. We denote this $(n-1)$-labeled Boolean algebra by $(\mathcal{C})_{n-1}$.

We observe that if $\mathcal{C}_{0} \leq_{n} \mathcal{C}_{1}$, then $\left(\mathcal{C}_{0}\right)_{n-1} \subseteq_{n-1}\left(\mathcal{C}_{1}\right)_{n-1}$, since $\alpha \leq_{n} \beta$ implies $(\alpha)_{n-1} \equiv_{n-1}(\beta)_{n-1}$. 
Definition 3.2. Let $\mathcal{B}$ be a an $n$-labeled Boolean algebra, $b \in \mathcal{B}$ and $\sigma=\mathfrak{t}_{n}^{\mathcal{B}}(b)$. We say that $b$ splits into a $\sigma$-full partition if there is a partition $b=\bigvee_{i=0}^{k} a_{i}$ in $\mathcal{B}$ such that

(1) $\mathfrak{t}_{n}^{\mathcal{B}}\left(a_{0}\right)=\sigma$, and

(2) for each $\delta \in \max \sigma$, there is some $j$ with $0<j \leq k$ such that $\mathfrak{t}_{n-1}^{\mathcal{B}}\left(a_{j}\right)=\delta$. (Recall from Remark 2.14 that $\max \sigma$ is well defined on $\equiv_{n}$-equivalence classes.)

Remark 3.3. For any Boolean algebra $\mathcal{B}$ and each $b \in \mathcal{B}$, if $T_{n}^{\mathcal{B}}(b)=\sigma$, then there always exists a $\sigma$-full partition of $b$. To see this, let $b=b_{0} \dot{V} \ldots \dot{\vee} b_{\ell}$ be a partition of $b$ into $n$-indecomposable elements (by Theorem 2.5(b)). It follows from Lemma 2.23 that we can further partition each $b_{i}$ to get a $T_{n}^{\mathcal{B}}\left(b_{i}\right)$-full partition of $b_{i}$. Let $b=a_{0} \dot{V} \ldots \dot{\vee} a_{k}$ be a partition of $b$ obtained by a $T_{n}^{\mathcal{B}}\left(b_{i}\right)$-full partition of each $b_{i}$. If $\delta \in \max \sigma$, then $\delta \in T_{n}^{\mathcal{B}}\left(b_{i}\right)$ for some $i$, so there is a $j \leq k$ with $T_{n-1}^{\mathcal{B}}\left(a_{j}\right)=\delta$.

3.2. $n$-approximations. We consider sequences of finite $n$-labeled Boolean algebras.

Definition 3.4. An $n$-approximation is a sequence $\left\{\left(\mathcal{A}_{k}, \mathfrak{t}_{n}^{k}\right)\right\}_{k \in \omega}$ of finite $n$-labeled Boolean algebras such that

(1) for each $k, \mathcal{A}_{k} \leq_{n} \mathcal{A}_{k+1}$;

(2) for each $k$ and every minimal element $b$ of $\mathcal{A}_{k}, b$ splits in $\mathcal{A}_{k+1}$ into a $\mathfrak{t}_{n}^{k+1}(b)$-full partition.

An $n$ - $Z$-approximation is an $n$-approximation that is computable in $Z$.

Remark 3.5. If $\left\{\left(\mathcal{A}_{k}, \mathrm{t}_{n}^{k}\right)\right\}_{k \in \omega}$ is an $n$-Z-approximation and if $m<n$, then $\left\{\left(\mathcal{A}_{k}\right.\right.$, $\left.\left.\mathfrak{t}_{m}^{k}\right)\right\}_{k \in \omega}$ is an $m$-Z-approximation, where $\mathfrak{t}_{m}^{k}(x)=\left(\ldots\left(\left(\mathfrak{t}_{n}^{k}(x)\right)_{n-1}\right) \ldots\right)_{m}$.

Theorem 3.6. Let $\left\{\left(\mathcal{A}_{k}, \mathrm{t}_{n}^{k}\right)\right\}_{k \in \omega}$ be an n-approximation and $\mathcal{A}=\bigcup_{k} \mathcal{A}_{k}$. Then for each $x \in \mathcal{A}$, the sequence $\left\{\mathfrak{t}_{n}^{k}(x)\right\}_{k \in \omega}$ eventually stabilizes and $T_{n}^{\mathcal{A}}(x)=\lim _{k \rightarrow \infty} \mathfrak{t}_{n}^{k}(x)$.

In this case we say that $\left\{\left(\mathcal{A}_{k}, \mathfrak{t}_{n}^{k}\right)\right\}_{k \in \omega}$ is an $n$-approximation of $\mathcal{A}$.

Proof. It is clear that $\mathcal{A}$ is a Boolean algebra. What we need to prove is that for every $a \in \mathcal{A}, T_{n}^{\mathcal{A}}(a)=\lim _{k \rightarrow \infty} \mathfrak{t}_{n}^{k}(a)$. That is, we need to show that the sequence $\left\{\mathfrak{t}_{n}^{k}(a)\right\}_{k \in \omega}$ stabilizes, and that once it stabilizes at a certain $n$-bf-type, it ends up building an element of that type. The fact that we are taking full partitions of every minimal element at every step guarantees the latter.

We prove, by induction on $n$, that for each element $a \in \mathcal{A}$, there exists a $k_{0} \in \omega$ such that $\forall k \geq k_{0}\left(\mathfrak{t}_{n}^{k}(a)=T_{n}^{\mathcal{A}}(a)\right)$. Since for every $k,\left(\mathcal{A}_{k}\right)_{n-1} \subseteq_{n-1}\left(\mathcal{A}_{k+1}\right)_{n-1}$, we may assume, by the induction hypothesis, that for every $x$ and sufficiently large $k, T_{n-1}^{\mathcal{A}}(x)=\mathfrak{t}_{n-1}^{k}(x)$. (In fact, $T_{n-1}^{\mathcal{A}}(x)=\mathfrak{t}_{n-1}^{k}(x)$ whenever $x \in \mathcal{A}_{k}$, since the value $\mathfrak{t}_{n-1}^{k}(x)$ does not change with $k$ once it is defined.)

First, we show that $\mathfrak{t}_{n}^{k}(a) \leq_{n} T_{n}^{\mathcal{A}}(a)$ for each $k$ by applying the following:

Given $\sigma \in \mathbf{I N V}_{n}$ and a Boolean algebra $\mathcal{B}$, we have that $\sigma \leq_{n}$ $T_{n}^{\mathcal{B}}\left(1_{\mathcal{B}}\right)$ if and only if for any partition $1_{\mathcal{B}}=b_{0} \dot{V} \ldots \dot{\vee} b_{\ell}$, there are invariants $\sigma_{0}, \ldots, \sigma_{\ell} \in \mathbf{I N V}_{n}$ with $\sigma=\sigma_{0}+\ldots+\sigma_{\ell}$ such that $T_{n-1}^{\mathcal{B}}\left(b_{i}\right) \leq_{n-1}\left(\sigma_{i}\right)_{n-1}$ for each $i \leq \ell$. (This follows from the equivalence $\mathcal{A}_{\sigma} \leq_{n} \mathcal{B}$ if and only if $\sigma \leq_{n} T_{n}^{\mathcal{B}}\left(1_{\mathcal{B}}\right)$.)

Let $a=a_{0} \dot{V} \ldots \dot{V} a_{\ell}$ be any partition of $a$ and let $k_{1} \geq k$ be such that $a_{0}, \ldots, a_{\ell} \in \mathcal{A}_{k_{1}}$ and $T_{n-1}^{\mathcal{A}}\left(a_{i}\right)=\mathfrak{t}_{n-1}^{k_{1}}\left(a_{i}\right)$ for each $i$ (using the induction hypothesis). Now, $\mathfrak{t}_{n}^{k_{1}}(a)=\mathfrak{t}_{n}^{k_{1}}\left(a_{0}\right)+\ldots+\mathfrak{t}_{n}^{k_{1}}\left(a_{\ell}\right)$ and $\mathfrak{t}_{n}^{k}(a) \leq_{n} \mathfrak{t}_{n}^{k_{1}}(a)$, so that, 
by Theorem 2.15(2), there exists $\sigma_{0}, \ldots, \sigma_{\ell} \in \mathbf{I N V}_{n}$ with $\mathfrak{t}_{n}^{k}(a)=\sigma_{0}+\ldots+\sigma_{\ell}$ and $\left(\sigma_{i}\right)_{n-1} \geq_{n-1} \mathfrak{t}_{n-1}^{k_{1}}\left(a_{i}\right)=T_{n-1}^{\mathcal{A}}\left(a_{i}\right)$ for each $i \leq \ell$. Thus, $\mathfrak{t}_{n}^{k}(a) \leq_{n} T_{n}^{\mathcal{A}}(a)$ by the condition above.

Next we show that for some $k_{0}, T_{n}^{\mathcal{A}}(a) \leq_{n} \mathfrak{t}_{n}^{k_{0}}(a)$, from which it follows that $T_{n}^{\mathcal{A}}(a) \equiv_{n} \mathfrak{t}_{n}^{k}(a)$ for all $k \geq k_{0}$ by the previous paragraph. It suffices to restrict the argument to the case where $T_{n}^{\mathcal{A}}(a) \in \mathbf{B F}_{n}$, since every element of $\mathcal{A}$ is a finite sum of $n$-indecomposables (by Theorem [2.5(b)), so that if $T_{n}^{\mathcal{A}}(a) \equiv_{n} \mathfrak{t}_{n}^{k}(a)$ for every $n$-indecomposable element $a$, the same holds true for every element of $\mathcal{A}$. Suppose that $T_{n}^{\mathcal{A}}(a)=\alpha$. Since the sequence $\mathfrak{t}_{n}^{k}(a)$ is $\leq_{n}$-increasing, it follows from Theorem 2.15(3) that for every $k, \operatorname{dc}\left(\mathfrak{t}_{n}^{k}(a)\right) \supseteq \operatorname{dc}\left(\mathfrak{t}_{n}^{k+1}(a)\right)$. Let $k_{0}$ be such that $\forall k \geq k_{0} \operatorname{dc}\left(\mathfrak{t}_{n}^{k}(a)\right)=\operatorname{dc}\left(\mathfrak{t}_{n}^{k_{0}}(a)\right)$ and let $a_{0}, \ldots, a_{\ell}$ be the minimal elements of $\mathcal{A}_{k_{0}}\left\lceil a\right.$, so that for each $i, \mathfrak{t}_{n}^{k_{0}}\left(a_{i}\right)=\beta_{i} \in \mathbf{B F}_{n}$ and $\beta_{0}+\ldots+\beta_{\ell}=\mathfrak{t}_{n}^{k_{0}}(a)$. We will prove that $\alpha \leq_{n} \beta_{0}+\ldots+\beta_{\ell}$ using the following, which is implied by Theorem 2.15(3):

Given $\alpha \in \mathbf{B F}_{n}$ and $\beta_{0}+\cdots+\beta_{\ell} \in \mathbf{I N V}_{n}$, we have that $\alpha \leq_{n}$ $\beta_{0}+\cdots+\beta_{\ell}$ if and only if

(1) $\operatorname{dc}\left(\beta_{0}+\cdots+\beta_{\ell}\right) \subseteq \operatorname{dc} \alpha$

(2) and there exist $\alpha_{0}, \ldots, \alpha_{\ell} \in \mathbf{I N V}_{n}$ with $\alpha=\alpha_{0}+\ldots+\alpha_{\ell}$ such that $\left(\beta_{i}\right)_{n-1} \leq_{n-1}\left(\alpha_{i}\right)_{n-1}$ for each $i \leq \ell$.

(11) For each $\delta \in \mathrm{dc}\left(\mathfrak{t}_{n}^{k_{0}}(a)\right)$, we claim that there are unboundedly many disjoint elements below $a$ of $(n-1)$-bf-type $\geq_{n-1} \delta$. Suppose not, and that $r \in \omega$ is maximal such that, for some $k>k_{0}$, there exist disjoint minimal elements $a_{0}, \ldots, a_{r}$ in $\mathcal{A}_{k}\lceil a$ with $\mathfrak{t}_{n-1}^{k}\left(a_{i}\right) \geq_{n-1} \delta$. Observe that for every $s>k$ and for each $i \leq r$, there is a minimal element $a_{i, s} \in \mathcal{A}_{s}\left\lceil a_{i}\right.$ with $\mathfrak{t}_{n-1}^{s}\left(a_{i, s}\right) \geq_{n-1} \delta$ (by Lemma 2.19 and that all the minimal elements below $a_{i}$ add up to $a_{i}$ ). Furthermore, there is at most one such $a_{i, s}$, since $r$ is maximal. This implies that $\delta \notin \mathrm{dc} \mathfrak{t}_{n}^{k+1}\left(a_{i}\right)$ for each $i \leq r$, otherwise a $\mathfrak{t}_{n}^{k+1}\left(a_{i}\right)$-full partition would add at least a second element of type $\geq_{n-1} \delta$ below $a_{i}$, contradicting the maximality of $r$. Let $b=a-\left(a_{0} \vee \cdots \vee a_{r}\right)$. Since $\delta \in \operatorname{dc}\left(\mathfrak{t}_{n}^{k+1}(a)\right)$, it follows that $\delta \in \mathrm{dc} \mathfrak{t}_{n}^{k+1}(b)$. But since $b$ splits into a $\mathfrak{t}_{n}^{k+1}(b)$-full partition, this would add an $(n-1)$-indecomposable bf-type $\geq_{n-1} \delta$ below $b$, contradicting the maximality of $r$. This proves our claim, and so $\delta \in \mathrm{dc} \alpha$.

(2) We have previously shown that $\beta_{i}=\mathfrak{t}_{n}^{k_{0}}\left(a_{i}\right) \leq_{n} T_{n}^{\mathcal{A}}\left(a_{i}\right)$, and thus $\left(\beta_{i}\right)_{n-1}$ $\equiv_{n-1} T_{n-1}^{\mathcal{A}}\left(a_{i}\right)$. So, $\alpha_{i}=T_{n}^{\mathcal{A}}\left(a_{i}\right)$ are as wanted.

Theorem 3.7. Let $\mathcal{A}$ be a presentation of a Boolean algebra, and let $Z$ be any set. The following are equivalent:

(1) $\mathcal{A}$ is n-Z-approximable. That is, $B_{n}(\mathcal{A})$ is $Z$-computable (Definition 2.9).

(2) $\mathcal{A}$ has an $n$-Z-approximation.

Proof. First, let us assume that $Z$ computes $\mathrm{B}_{n}(\mathcal{A})$. We will define an $n$ - $Z$ approximation $\left\{\left(\mathcal{A}_{k}, \mathfrak{t}_{n}^{k}\right)\right\}_{k \in \omega}$ of $\mathcal{A}$. Let $u$ be the partial function on $\mathcal{A}$ defined so that, for each $a \in \mathcal{A}, u(a)$ is the $\leq_{n}$-greatest $n$-bf-type $\gamma \in \mathbf{B F}_{n}$ such that $\mathrm{R}_{\gamma}(a)$ holds in $\mathcal{A}$, if such $\gamma$ exists, and $u(a)$ is undefined otherwise. Note that the function $u$ and its domain are $Z$-computable and that $u(a) \leq_{n} T_{n}^{\mathcal{A}}(a)$; thus, for $n$-indecomposable $a \in \mathcal{A}$, we have $u(a)=T_{n}^{\mathcal{A}}(a)$.

To define $\mathcal{A}_{0}$, search for a partition $a_{0}, \ldots, a_{\ell}$ of $1_{\mathcal{A}}$ such that for every $i, u\left(a_{i}\right)$ is defined (which must exist by Theorem 2.5). Let $\mathcal{A}_{0}$ be the Boolean algebra generated by these $a_{i}$, and let $\mathfrak{t}_{n}^{0}\left(a_{i}\right)=u\left(a_{i}\right)$. 
Let $\left\{b_{0}, b_{1}, \ldots\right\}$ be an enumeration of the non-zero elements of $\mathcal{A}$. To ensure that $\mathcal{A}=\bigcup_{k \in \omega} \mathcal{A}_{k}$, we will make sure that $b_{k} \in \mathcal{A}_{k+1}$. Suppose we have already defined $\left(\mathcal{A}_{k}, \mathfrak{t}_{n}^{k}\right)$ so that $\left\{b_{0}, \ldots, b_{k-1}\right\} \subseteq \mathcal{A}_{k}$. For each minimal element $a$ of $\mathcal{A}_{k}$, look for a partition $a_{0}, \ldots, a_{\ell} \in \mathcal{A}$ of $a$ such that

(a) $u\left(a_{i}\right)$ is defined for each $i \leq \ell$;

(b) for some $h \leq \ell, a \wedge b_{k}=\bigvee_{i=0}^{h} a_{i}$ and $a-b_{k}=\bigvee_{i=h+1}^{\ell} a_{i}$;

(c) $\mathfrak{t}_{n}^{k}(a) \leq_{n} \sum_{i=0}^{\ell} u\left(a_{i}\right)$;

(d) the elements $a_{0}, \ldots, a_{\ell}$ form a $\left(\sum_{i=0}^{\ell} u\left(a_{i}\right)\right)$-full partition of $a$.

By Remark 3.3 there is a partition $a_{0}, \ldots, a_{h}$ of $a \wedge b_{k}$ into $n$-indecomposable elements and a partition $a_{h+1}, \ldots, a_{\ell}$ of $a-b_{k}$ into $n$-indecomposable so that $a_{0}, \ldots, a_{\ell}$ is a $\left(\sum_{i=0}^{\ell} T_{n}^{\mathcal{A}}\left(a_{i}\right)\right)$-full partition of $a$ (conditions (b) and $\left.(\mathrm{d})\right)$. Since these elements are $n$-indecomposable, $u\left(a_{i}\right)$ is defined and $u\left(a_{i}\right) \equiv_{n} T_{n}^{\mathcal{A}}\left(a_{i}\right)$ (condition (a)). Finally, condition (c) follows from the fact that $a$ is minimal:

$$
\mathfrak{t}_{n}^{k}(a)=u(a) \leq_{n} T_{n}^{\mathcal{A}}(a) \equiv_{n} \sum_{i=0}^{\ell} T_{n}^{\mathcal{A}}\left(a_{i}\right) \equiv_{n} \sum_{i=0}^{\ell} u\left(a_{i}\right) .
$$

Thus, a $Z$-computable search will eventually find such a partition of $a$ which satisfies these four conditions. (Although we note that since we are using the predicates $\mathrm{R}_{\gamma}$ to find these elements, they need not be $n$-indecomposable.)

Let $\mathcal{A}_{k+1}$ be the extension of $\mathcal{A}_{k}$ generated by the partitions $a_{0}, \ldots, a_{\ell}$ chosen below each minimal element $a$ of $\mathcal{A}_{k}$. For each of these new elements $a_{i}$, let $\mathfrak{t}_{n}^{k+1}\left(a_{i}\right)=u\left(a_{i}\right)$. For each $b \in \mathcal{A}_{k+1}$ with $b=\bigvee_{i=1}^{j} c_{i}$, where the $c_{i}$ 's are minimal elements in $\mathcal{A}_{k+1}$, let $\mathfrak{t}_{n}^{k+1}(b)=\sum_{i=1}^{j} u\left(c_{i}\right)$.

For every minimal element $a \in \mathcal{A}_{k}$, we have $\mathfrak{t}_{n}^{k}(a) \leq_{n} \mathfrak{t}_{n}^{k+1}(a)$, and since $\left(\mathbf{I N V}_{n}\right.$, ,$\left.+ \leq_{n}\right)$ is an ordered monoid, we can extend this to $\left(\mathcal{A}_{k}, \mathfrak{t}_{n}^{k}\right) \leq_{n}\left(\mathcal{A}_{k+1}, \mathfrak{t}_{n}^{k+1}\right)$. Furthermore, $b_{k} \in \mathcal{A}_{k+1}$ since both $b_{k} \wedge a$ and $a-b_{k}$ are in $\mathcal{A}_{k+1}$. This completes the proof of (2).

Suppose now that we have an $n$-Z-approximation $\left\{\left(\mathcal{A}_{k}, \mathfrak{t}_{n}^{k}\right)\right\}_{k \in \omega}$ of $\mathcal{A}$. By Remark [3.5, we have $m$ - $Z$-approximations of $\mathcal{A}$ for $m \leq n$ as well. Furthermore, for $m<n$, and for all $\alpha \in \mathbf{B F}_{m}$ and $a \in \mathcal{A}$, we have that $\mathrm{R}_{\alpha}(a)$ if and only if $\mathfrak{t}_{m}^{k}(a) \geq_{m} \alpha$, where $k$ is such that $a \in \mathcal{A}_{k}$. This is because for all $m<n$, $\mathfrak{t}_{m}^{k}(a)=T_{m}^{\mathcal{A}}(a)$. Thus, the predicates $\mathrm{R}_{\beta}$ are computable in $Z$ for $\beta \in \mathbf{B F}_{n-1}$. Given $a \in \mathcal{A}$, we have that $\mathrm{R}_{\alpha}(a)$ if and only if $\exists k\left(\mathfrak{t}_{n}^{k}(a) \geq_{n} \alpha\right)$, so all the predicates $\mathrm{R}_{\alpha}(\mathcal{A})$ for $\alpha \in \mathbf{B F}_{n}$ are computably enumerable in $Z$. On the other hand, from the inductive step in the proof of [HM] Lemma 8.10], we get that $\mathrm{R}_{\alpha}$ is $\Pi_{1}^{c}$ over the predicates $\mathbf{R}_{\beta}$ for $\beta \in \mathbf{B F}_{n-1}$. Since the predicates $\mathbf{R}_{\beta}$ for $\beta \in \mathbf{B F}_{n-1}$ are $Z$-computable, it follows that $\mathrm{R}_{\alpha}(\mathcal{A})$ is co-c.e. in $Z$. Thus, the predicates $\mathrm{R}_{\alpha}(\mathcal{A})$ for $\alpha \in \mathbf{B F}_{n}$ are computable in $Z$.

Remark 3.8. The proof of Theorem 3.7 is uniform in the following sense. We can uniformly go from a $Z$-computable index for $\mathrm{B}_{n}(\mathcal{A})$ to a $Z$-computable index for the sequence $\left\{\left(\mathcal{A}_{k}, \mathrm{t}_{n}^{k}\right)\right\}_{k \in \omega}$, and vice versa. When we refer to an index for an $n$ - $Z$-approximable Boolean algebra, we mean an index of either of these two kinds.

\section{Some USEFul 5-BF-TyPes}

We now describe the 5-indecomposable types we are going to use in our construction. All the 5-bf-types are described in [HM, Section 6]. There are two 
5-indecomposable types that are the principal actors in our construction: $w=f_{23}$ and $u=f_{24}$. The key is that both $u$ and $w$ are exclusive 5-indecomposable types (Definition 2.17) and $w<_{5} u$, and, in fact, $u$ is the only such bf-type. No such pair of exclusive $n$-indecomposable types exists for $n=1,2,3,4$. Another key actor is $w^{\infty}=f_{12}$, which is not exclusive. The relationship of $w^{\infty}$ to $w$ and $u$ is given by

$$
w^{\infty}<_{5} w<_{5} u
$$

These three 5-bf-types have the same parent, which we call $e_{6} \in \mathbf{B F}_{4}$. In other words, $(u)_{4}=(w)_{4}=\left(w^{\infty}\right)_{4}=e_{6}$. The 5-approximable Boolean algebras we construct will use only seven of the twenty-seven 5 -indecomposable types: $f_{0}, f_{1}, f_{2}, f_{12}$, $f_{23}, f_{24}, f_{26}$. Let

$$
D=\left\{f_{0}, f_{1}, f_{2}, f_{12}, f_{23}, f_{24}, f_{26}\right\} \subset \mathbf{B F}_{5},
$$

and let $\langle D\rangle=\left\{\sum_{i=0}^{k} \beta_{i}: k \in \omega, \beta_{i} \in D\right\} \subseteq \mathbf{I N V}_{5}$, the subset of $\mathbf{I N V}_{5}$ generated by $D$ under addition. The following five members of $D$ have the property that all Boolean algebras having their type are isomorphic: $f_{0}$ corresponds to atoms, $f_{1}$ to 1 -atoms, $f_{2}$ to 2 -atoms, $f_{26}$ to the atomless Boolean algebra, and $u=f_{24}$ to the interval algebra Int $\left(\omega^{2}+\eta\right)$. We will say that such back-and-forth types are isomorphism types. The other two 5-bf-types are not isomorphism types, but in our construction we will consider only one Boolean algebra of type $w=f_{23}$, namely, the interval algebra $\operatorname{Int}\left(\omega^{3}+\eta\right)$. We will also consider only two Boolean algebras of type $w^{\infty}=f_{12}$ : $\operatorname{Int}\left(\left(\omega^{3}+\eta\right) \cdot \omega\right)$, which we call a 1 - $w$-atom and has the property that in any partition of the algebra exactly one element is of the type $w^{\infty}$, and $\operatorname{Int}\left(\left(\omega^{2}+1+\eta\right) \cdot \eta\right)$, which has densely many elements of type $w^{\infty}$.

We are going to build a sequence $\{\mathcal{A}[s]\}_{s \in \omega}$ of finite 5 -labeled Boolean algebras with the following properties:

(D1) All the minimal elements of $\mathcal{A}[s]$ are labeled with types in $D$.

(D2) Elements of types $f_{0}, f_{1}, f_{2}, f_{26}$, and $u$ never change their type. Minimal elements in $\mathcal{A}[s]$ of 5 -indecomposable types $w^{\infty}$ can either retain type $w^{\infty}$ or increase their type to $w$ in $\mathcal{A}[s+1]$. Minimal elements of type $w$ can either retain type $w$ or increase their type to $u$.

(D3) Each minimal element $a$ of $\mathcal{A}[s]$ splits in $\mathcal{A}[s+1]$ into a canonical $\mathfrak{t}_{5}^{\mathcal{A}[s+1]}(a)$ full partition as indicated in the table below.

(D4) $\mathfrak{t}_{5}^{\mathcal{A}[s]}(1)=w^{\infty}$ for all $s$.

Note that these conditions guarantee that $\{\mathcal{A}[s]\}_{s \in \omega}$ is a 5 -approximation of some Boolean algebra $\mathcal{A}$ with $T_{5}^{\mathcal{A}}\left(1_{\mathcal{A}}\right)=w^{\infty}$.

In our construction, we will use a particular kind of full partition (Definition 3.2) that we call a canonical partition and we list below. To each $\gamma \in D$, we provide a list $\gamma_{0}, \ldots, \gamma_{k}$ of 5 -indecomposable bf-types such that $\gamma \equiv_{5} \sum_{i \leq k} \gamma_{i}, \gamma_{0}=\gamma$, and for each $i$ with $1 \leq i \leq k,\left(\gamma_{i}\right)_{4} \in \gamma$. The point is that if $a \in \mathcal{A}[s], \mathfrak{t}_{5}^{\mathcal{A}[s+1]}(a)=\gamma$ and $a$ is minimal in $\mathcal{A}[s]$, then $a$ partitions into $a=b_{0} \dot{\vee} \ldots \dot{\vee} b_{k}$ in $\mathcal{A}[s+1]$ with $\mathfrak{t}_{5}^{\mathcal{A}[s+1]}\left(b_{i}\right)=\gamma_{i}$ for each $i \leq k$. To help the reader picture these types, in the final column we note the isomorphism type of the algebra constructed below an element of a given type, provided the type of the element does not change during the construction. 


\begin{tabular}{|c|l|c|}
\hline type & canonical partition & algebra \\
\hline$f_{0}$ & $f_{0}$ & $\operatorname{Int}(2)$ \\
$f_{1}$ & $f_{1}, f_{0}$ & $\operatorname{Int}(\omega)$ \\
$f_{2}$ & $f_{2}, f_{0}, f_{1}$ & $\operatorname{Int}\left(\omega^{2}\right)$ \\
$f_{26}$ & $f_{26}, f_{26}$ & $\operatorname{Int}(\eta)$ \\
$u=f_{24}$ & $u, f_{0}, f_{1}, f_{26}$ & $\operatorname{Int}\left(\omega^{2}+\eta\right)$ \\
$w=f_{23}$ & $w, f_{0}, f_{1}, f_{2}, f_{26}$ & $\operatorname{Int}\left(\omega^{3}+\eta\right)$ \\
$w^{\infty}=f_{12}$ & $w^{\infty}, w^{\infty}, f_{0}, f_{1}, f_{2}, f_{26}$ & - \\
\hline
\end{tabular}

The next theorem describes $\left(\langle D\rangle, \leq_{5},+\right)$. A key fact is that the only 5 -indecomposable type in $D$ which has $e_{6}$ in its downward closure is $w^{\infty}$, as can be seen in the description of the 5-indecomposable types of $D$ from Section 6 of [HM].

Theorem 4.1. The following equations hold in $\mathbf{I N} \boldsymbol{V}_{5}$ :

(1) $u=u+f_{0}=u+f_{1}=u+f_{26}$.

(2) $w=w+f_{0}=w+f_{1}=w+f_{2}=w+f_{26}$.

(3) $w^{\infty}=w^{\infty}+f_{0}=w^{\infty}+f_{1}=w^{\infty}+f_{2}=w^{\infty}+f_{26}=w^{\infty}+u=w^{\infty}+w=$ $w^{\infty}+w^{\infty}$.

(4) If $\sigma \in\langle D\rangle$ and $\sigma \equiv_{5} \beta \in \boldsymbol{B F}_{5}$, then $\beta \in D$.

(5) Let $\sigma=\beta_{0}+\ldots+\beta_{k} \in\langle D\rangle$.

(a) If $\sigma \equiv_{5} u$, then there is exactly one $i$ with $\beta_{i}=u$ and $\beta_{j} \in\left\{f_{0}, f_{1}, f_{26}\right\}$ for all $j \neq i$.

(b) If $\sigma \equiv_{5} w$, then there is exactly one $i$ with $\beta_{i}=w$ and $\beta_{j} \in\left\{f_{0}, f_{1}, f_{2}\right.$, $\left.f_{26}\right\}$ for all $j \neq i$.

(c) If $\sigma \equiv_{5} w^{\infty}$, then there is some $i$ with $\beta_{i}=w^{\infty}$.

Proof. We can use Lemma 2.20 to show each of (11)-(3). We let the reader verify (using the material in [HM, Section 6]) that $u \triangleleft_{5} f_{0}, f_{1}, f_{26}$, that $w \triangleleft_{5} f_{0}, f_{1}, f_{2}, f_{26}$, and that $w^{\infty} \triangleleft_{5} f_{0}, f_{1}, f_{2}, f_{26}, u, w$. For instance, to verify that $w^{\infty} \triangleleft_{5} u$, we need to note that $u=\left\{e_{0}, e_{1}, e_{8}\right\} \subseteq\left\{e_{0}, e_{1}, e_{2}, e_{6}, e_{8}\right\}=\operatorname{dc} w^{\infty}$, and that $(u)_{4}=e_{6} \in$ $\operatorname{dc} w^{\infty}$. Then, by Lemma 2.20, this implies that $w^{\infty}=w^{\infty}+u$.

Before we prove the next two parts, we make a general point about $n$-indecomposable invariants. Suppose that $\sigma \equiv_{n} \beta \in \mathbf{B F}_{n}$ and $\sigma=\delta+\gamma+\rho$ (where $\delta, \gamma \in \mathbf{B F}_{n}$ ). Then $\beta \leq_{n} \delta+\gamma+\rho$, so that by Lemma 2.19 either $\beta \triangleleft_{n} \delta$ or $\beta \triangleleft_{n} \gamma$. It then follows from Definition 2.18 that either $(\delta)_{n-1} \in \operatorname{dc} \beta$ or $(\gamma)_{n-1} \in \operatorname{dc} \beta$, and since $\operatorname{dc} \beta=\operatorname{dc} \sigma$ (from Remark 2.14), we have that either $(\delta)_{n-1} \in \operatorname{dc} \sigma$ or $(\gamma)_{n-1} \in \operatorname{dc} \sigma$.

For part (4): suppose that $\sigma \in\langle D\rangle$ and $\sigma \equiv_{5} \beta \in \mathbf{B F}_{5}$. If $\sigma=w^{\infty}+\tau$, where $\tau \in\langle D\rangle$, then $\sigma \equiv_{5} w^{\infty}$ by (3). Suppose $\sigma \not_{5} w^{\infty}$, but is a sum built from $w$ or $u$. It follows that $e_{6} \notin \mathrm{dc} \sigma$, since $w^{\infty}$ is the only element of $D$ which has $e_{6}$ in its downward closure. Since $\sigma$ is a 5 -indecomposable bf-type, it cannot be of the form $w+w+\rho, w+u+\rho$ or $u+u+\rho$, as none of these are 5 -indecomposable bf-types by the previous paragraph. If $\sigma=w+\tau$, then $\tau \in\left\langle\left\{f_{0}, f_{1}, f_{2}, f_{26}\right\}\right\rangle$, and thus $\sigma \equiv_{5} w$ by (2). If $\sigma=u+\tau$, then since the only members of $D$ which have $\left(f_{2}\right)_{4}$ in their downward closure are $w$ and $w^{\infty}$, we must have $\tau \in\left\langle\left\{f_{0}, f_{1}, f_{26}\right\}\right\rangle$. Thus, $\sigma \equiv_{5} u$ by (11). The remaining possibility is that $\sigma \in\left\langle\left\{f_{0}, f_{1}, f_{2}, f_{26}\right\}\right\rangle$. Recall that $f_{0}$ is an atom, $f_{1}$ is a 1 -atom, $f_{2}$ is a 2 -atom and $f_{26}$ is the atomless type. Since $\sigma \equiv_{5} \beta$, it follows by Lemma 2.19 that there is a $\delta \in\left\{f_{0}, f_{1}, f_{2}, f_{26}\right\}$ with $\sigma=\delta+\tau$ and $\beta \leq_{5} \delta$. If $\delta \in\left\{f_{0}, f_{1}, f_{26}\right\}$, then $\beta=\delta$ since there is nothing strictly $\leq_{5}$ of these bf-types; they are all descended from isomorphism types at levels below 5 . The only 5-bf-types satisfying $\beta<_{5} f_{2}$ have $\left(f_{2}\right)_{4} \in \beta$ (which can be verified in 
[HM, Section 6$]$ ), but no 5 -bf-type in the set $\left\{f_{0}, f_{1}, f_{2}, f_{26}\right\}$ has this property. So, $\beta \leq_{5} f_{2}$ implies that $\beta=f_{2}$.

For part (5), suppose that $\sigma=\beta_{0}+\ldots+\beta_{k} \in\langle D\rangle$. Recall that $w^{\infty}<_{5} w<_{5} u$. We will apply Lemma 2.19 in each case. If $\sigma \equiv_{5} u$, then $u \leq_{5} \beta_{0}+\ldots+\beta_{k}$ so that for some $i, u \leq_{5} \beta_{i}$, and thus $u=\beta_{i}$, and $u \triangleleft_{5} \beta_{j}$ for each $j \neq i$, so that $u+\beta_{j} \equiv_{5} u$ (by Lemma 2.20) implying $\beta_{j} \in\left\{f_{0}, f_{1}, f_{26}\right\}$ by (11). If $\sigma \equiv_{5} w$, a similar argument shows that $w \leq_{5} \beta_{i}$ for some $i$ and $\beta_{j} \in\left\{f_{0}, f_{1}, f_{2}, f_{26}\right\}$ for each $j \neq i$. We will now show that $\left(f_{2}\right)_{4} \in \mathrm{dc} \beta_{i}$ from which it follows that $\beta_{i}=w$. Since $\sigma \equiv_{5} w$ and $\left(f_{2}\right)_{4} \in \mathrm{dc} w$, we have $\left(f_{2}\right)_{4} \in \mathrm{dc} \cup_{j} \beta_{j}$, and so $\left(f_{2}\right)_{4} \in \operatorname{dc} \beta_{j}$ for some $j$ (by Remark 2.14) and in fact $\left(f_{2}\right)_{4} \in \mathrm{dc} \beta_{i}$, since $\left(f_{2}\right)_{4}$ is not in the downward closure for any of $f_{0}, f_{1}, f_{2}$ or $f_{26}$. If $\sigma \equiv_{5} w^{\infty}$, then since $e_{6} \in \mathrm{dc} w^{\infty}$, we must also have $e_{6} \in \mathrm{dc} \cup_{j} \beta_{j}$, and so $e_{6} \in \mathrm{dc} \beta_{i}$ for some $i$ (by Remark 2.14). Since $w^{\infty}$ is the unique 5-bf-type in $D$ with $e_{6} \in \mathrm{dc} w^{\infty}$, we must have at least one $i$ with $\beta_{i}=w^{\infty}$.

Definition 4.2. Let $\mathcal{A}$ be a Boolean algebra and $\gamma$ an $n$-indecomposable type. We let $\operatorname{num}(\gamma, \mathcal{A}) \in \omega \cup\{\infty\}$ be the maximum $r$ such that $1_{\mathcal{A}}=a_{0} \dot{\vee} a_{1} \dot{V} \ldots \dot{\vee} a_{r}$, where each $\mathcal{A}\left\lceil a_{i}\right.$ (for $1 \leq i \leq r$ ) is $n$-indecomposable and $t_{n}\left(a_{i}\right)=\gamma$.

For a finite $n$-labeled Boolean algebra $\mathcal{A}$, we let $\operatorname{num}(\gamma, \mathcal{A})$ be the number of minimal elements of $\mathcal{A}$ of type $\gamma$.

Definition 4.3. A $1-w$-atom is a Boolean algebra $\mathcal{A}$ that satisfies the following properties:

(i) $T_{5}(\mathcal{A})=w^{\infty}$

(ii) For each 5-indecomposable element $a \in \mathcal{A}$, if $T_{4}^{\mathcal{A}}(a)=e_{6}$, then $T_{5}^{\mathcal{A}}(a) \in$ $\left\{w, w^{\infty}\right\}$.

(iii) There is no disjoint pair $a, b \in \mathcal{A}$ with $\operatorname{num}\left(e_{6}, \mathcal{A}\lceil a)=\operatorname{num}\left(e_{6}, \mathcal{A}\lceil b)=\infty\right.\right.$.

Lemma 4.4. Let $\{\mathcal{A}[s]\}_{s \in \omega}$ be a 5-approximation of a Boolean algebra $\mathcal{A}$ that satisfies (D1)-(D4). Suppose that for each $s_{0}$ :

(i) No minimal element of $\mathcal{A}\left[s_{0}\right]$ is labeled with the 5-indecomposable type $u$.

(ii) There is a stage $s \geq s_{0}$ such that there remains exactly one minimal element $a \in \mathcal{A}\left[s_{0}\right]$ with $\mathfrak{t}_{5}^{\mathcal{A}[s]}(a)=w^{\infty}$.

Then $\mathcal{A}$ is a 1-w-atom.

Proof. By condition (D4), $T_{5}(\mathcal{A})=w^{\infty}$. By condition (D1) and Theorem 4.1(4), every 5 -indecomposable element $a \in \mathcal{A}$ with $T_{4}^{\mathcal{A}}(a)=e_{6}$ must satisfy $T_{5}^{\mathcal{A}}(a) \in$ $\left\{u, w, w^{\infty}\right\}$. We now show that $T_{5}^{\mathcal{A}}(a) \in\left\{w, w^{\infty}\right\}$. It follows from Theorem 4.1 (5) that if $\sigma \in\langle D\rangle$ and $\sigma \equiv_{5} u$, then $\sigma=u+\tau$ for some $\tau \in\left\langle f_{0}, f_{1}, f_{26}\right\rangle$. If $T_{5}^{\mathcal{A}}(a)=u$, then by Theorem 3.6. there is an $s_{0}$ with $\mathfrak{t}_{5}^{A\left[s_{0}\right]}(a)=u$, so that for some minimal element $b \leq a, \mathfrak{t}_{5}^{A\left[s_{0}\right]}(b)=u$, contradicting assumption (i) of the lemma. Thus, $T_{5}^{\mathcal{A}}(a) \in\left\{w, w^{\infty}\right\}$. This establishes the first two conditions of Definition 4.3.

Now we will show that any $a \in \mathcal{A}$ with $\operatorname{num}\left(e_{6}, \mathcal{A}\lceil a)=\infty\right.$ is 5 -indecomposable and $T_{5}^{\mathcal{A}}(a)=w^{\infty}$. Since $e_{6} \in \operatorname{dc} T_{5}^{\mathcal{A}}(a)$, it follows from condition (D1) that $T_{5}^{\mathcal{A}}(a)=$ $w^{\infty}+\tau$, where $\tau \in\langle D\rangle$, and so $T_{5}^{\mathcal{A}}(a)=w^{\infty}$ by Theorem 4.1(3). For any partition $a=a_{0} \dot{V} \ldots \dot{\vee} a_{k}$ there must be some $i \leq k$ with $\operatorname{num}\left(e_{6}, \mathcal{A}\left\lceil a_{i}\right)=\infty\right.$, so that $T_{5}^{\mathcal{A}}\left(a_{i}\right)=w^{\infty}$, and thus $\mathcal{A}\left\lceil a \equiv_{5} \mathcal{A}\left\lceil a_{i}\right.\right.$.

Finally, suppose there were disjoint elements $a, b \in \mathcal{A}$ with $\operatorname{num}\left(e_{6}, \mathcal{A}\lceil a)=\infty\right.$ and $\operatorname{num}\left(e_{6}, \mathcal{A}\lceil b)=\infty\right.$ and let $s_{0}$ be a stage in which $a, b \in \mathcal{A}\left[s_{0}\right]$. For each $s \geq s_{0}$, there must be minimal $a^{\prime} \leq a$ and $b^{\prime} \leq b$ in $\mathcal{A}[s]$ with $\operatorname{num}\left(e_{6}, \mathcal{A}\left\lceil a^{\prime}\right)=\infty\right.$ 
and $\operatorname{num}\left(e_{6}, \mathcal{A}\left\lceil b^{\prime}\right)=\infty\right.$. From the previous paragraph, $T_{5}^{\mathcal{A}}\left(a^{\prime}\right)=w^{\infty}=T_{5}^{\mathcal{A}}\left(b^{\prime}\right)$, but since $\mathfrak{t}_{5}^{\mathcal{A}[s]}\left(a^{\prime}\right) \leq_{5} T_{5}^{\mathcal{A}}\left(a^{\prime}\right)$ and $\mathfrak{t}_{5}^{\mathcal{A}[s]}\left(b^{\prime}\right) \leq_{5} T_{5}^{\mathcal{A}}\left(b^{\prime}\right)$, it follows from (D1) and the minimality of $w^{\infty}$ in $D$ that $\mathfrak{t}_{5}^{\mathcal{A}[s]}\left(a^{\prime}\right)=w^{\infty}=\mathfrak{t}_{5}^{\mathcal{A}[s]}\left(b^{\prime}\right)$, in contradiction to assumption (ii) of the lemma. This establishes the third condition of Definition 4.3 .

In fact, the algebra $\mathcal{A}$ constructed in the previous lemma is isomorphic to the interval algebra $\operatorname{Int}\left(\left(\omega^{3}+\eta\right) \cdot \omega\right)$ (although we will not use this fact in what follows).

4.1. Picturing $n$-approximations. A good way to picture $n$-approximations is by the use of labeled trees. Let $\{\mathcal{A}[s]\}_{s \in \omega}$ be an $n$-approximation of an algebra $\mathcal{A}$. At the root of the tree, we put one node labeled $\mathfrak{t}_{n}^{\mathcal{A}[0]}\left(1_{\mathcal{A}}\right)$. At the $s$ th level of the tree, we draw the minimal elements of $\mathcal{A}[s]$ and label them using $\mathfrak{t}_{n}^{\mathcal{A}[s]}$. The ordering on the nodes of the tree comes from the ordering of the minimal elements of $\mathcal{A}[s+1]$ relative to those of $\mathcal{A}[s]$. So, the labels of the children at level $(s+1)$, of a node $a$ at level $s$, form a $\mathfrak{t}_{n}^{\mathcal{A}[s+1]}(a)$-full partition. Notice that the label of $a$ does not need to be $\mathfrak{t}_{n}^{\mathcal{A}[s+1]}(a)$, but $\mathfrak{t}_{n}^{\mathcal{A}[s]}(a)$.

In the picture below we draw the first few levels of the trees corresponding to 5-approximations of the Boolean algebras $\mathcal{A}_{f_{1}}, \mathcal{A}_{f_{26}}$, and $\mathcal{A}_{u}$, which are of types $f_{1}, f_{26}$, and $u$, respectively. All of these examples satisfy (D1)-(D4), and the 5-bftypes of the elements do not change over time.
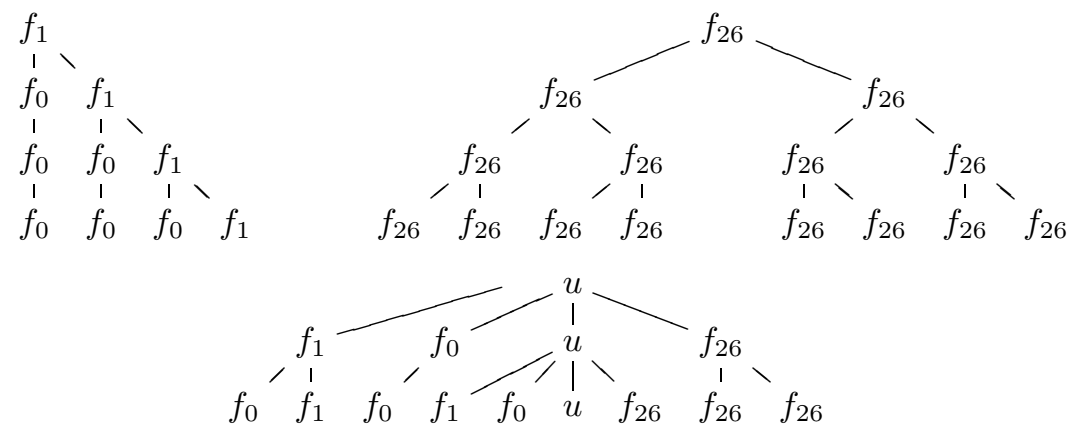

\section{NeCESSARY TOOLS}

In this section we establish several lemmas that will be useful in our construction. First, we look at how to deal with guesses of answers to $0^{\prime \prime}$ questions. Second, we consider infinite sums of $n$ - $Z$-approximable Boolean algebras. Third, we show how to enumerate all the 4-0-approximable Boolean algebras, recursively in $0^{\prime}$. The reader might want to skip the technical proofs in this section in a first read of the paper.

5.1. Zero double guesses. The following lemma shows that the guesses to $0^{\prime \prime}$ questions can be ordered so that the correct one is the limit infimum of all the guesses. This is not new and is essentially what happens when one does an infiniteinjury priority argument on a tree of strategies.

Lemma 5.1. Given $e \in \omega$, there are total computable functions $z: \omega \rightarrow \omega$ and $g: \omega \rightarrow \omega$ such that there is at most one $\ell$ with $z(s)=\ell$ for infinitely many $s$. Moreover, there is an $\ell_{0}$ with $z(s)=\ell_{0}$ for infinitely many $s$ if and only if $\varphi_{e}^{0^{\prime \prime}}(0) \downarrow$, in which case $\varphi_{e}^{0^{\prime \prime}}(0)=g\left(\ell_{0}\right)$. 
Remark 5.2. We can construct $z$ and $g$ so that, also, for every $\ell$, if $z(s)<\ell \leq s$, then $z(t) \neq \ell$ for all $t \geq s$. Thus, if $\varphi_{e}^{0^{\prime \prime}}(0)=g\left(\ell_{0}\right)$, then $\ell_{0} \leq z(s)$ for all $s \geq \ell_{0}$.

Proof. Let $K=0^{\prime}$ and $Z=K^{\prime}=0^{\prime \prime}$. Fix a computable enumeration $\left\{k_{0}, k_{1}, \ldots\right\}$ of $K$. We construct a computable approximation of $K$ using finite strings $\left\{K_{s}\right.$ : $s \in \omega\}: K_{s} \in\{0,1\}^{k_{s}+1}$ satisfying $K_{s}(x)=1$ if and only if $x=k_{i} \leq k_{s}$ for some $i \leq s$. We say that a stage $t$ is a true stage if $\forall s>t\left(k_{s}>k_{t}\right)$; thus, $t$ is a true stage if and only if $K_{t}=K\left\lceil k_{t}+1\right.$, and also if and only if $K_{t} \subseteq K_{s}$ for all $s \geq t$ (where "ᄃ" means an initial segment). Thus, $K=\bigcup_{t}$ a true stage $K_{t}$ (where the union is of partial functions into $\{0,1\})$.

Similarly, we construct a computable approximation $\left\{Z_{s}\right\}_{s \in \omega}$ of $Z$ so that $Z=$ $\lim _{t}$ a true stage $Z_{t}$ : Given a string $\sigma \in\{0,1\}^{<\omega}$ and $x<|\sigma|$, we define

$$
\sigma^{\prime}(x)= \begin{cases}1 & \text { if } \varphi_{x,|\sigma|}^{\sigma}(x) \downarrow \\ 0 & \text { otherwise }\end{cases}
$$

(where $\varphi_{x}^{\sigma}$ is the $x$ th Turing functional) and take $Z_{s}=K_{s}^{\prime}$. If $Z(x)=1$, then there exists a finite string $\sigma \subseteq K$ with $\sigma^{\prime}(x)=1$ and a true stage $t$ with $\sigma \subseteq K_{t}$; in this case $Z_{s}(x)=1$ for all $s \geq t$. If $Z(x)=0$, then $K_{t}^{\prime}(x)=0$ for every true stage $t$ with $x<\left|K_{t}^{\prime}\right|$. Thus, $Z=\lim _{t}$ a true stage $K_{t}^{\prime}$.

We now define $g$ and $z$ as follows. Let

$$
g(s)=\varphi_{e, s}^{Z_{s}}(0)
$$

and $g(s)=0$ if this computation does not converge. If $\varphi_{e, s}^{Z_{s}}(0) \downarrow$, then let $\tilde{Z}_{s}$ (the use of this computation) be the shortest initial segment of $Z_{s}$ such that $\varphi_{e, s}^{\tilde{Z}_{s}}(0) \downarrow$, and otherwise let $\tilde{Z}_{s}=Z_{s}$ (i.e. if $\left.\varphi_{e, s}^{Z_{s}}(0) \uparrow\right)$. Let $\tilde{K}_{s}$ be the shortest initial segment of $K_{s}$ such that $\tilde{K}_{s}^{\prime} \supseteq \tilde{Z}_{s}$. We define $z(s)$ to be the least $t \leq s$ such that $K_{t} \subseteq K_{s}$ and $\tilde{K}_{t}=\tilde{K}_{s}$, and if $t<s$, then $\varphi_{e, t}^{Z_{t}}(0) \downarrow$. Note that $g(s)=g(z(s))$ because $\tilde{Z}_{s}=\tilde{Z}_{z(s)}$.

We show that $g$ and $z$ are as claimed. Suppose $z(s)=\ell_{0}$ for infinitely many $s$. Then $\ell_{0}$ is a true stage, since $K_{\ell_{0}} \subseteq K_{s}$ for infinitely many $s$. We now claim that $\tilde{Z}_{\ell_{0}} \subseteq Z$. If not, then since $\ell_{0}$ is a true stage, there is some $x$ such that $\tilde{Z}_{\ell_{0}}(x)=0$ and $Z(x)=1$. In this case there would be another true stage $t>\ell_{0}$ with $Z_{t}(x)=1$. But now for each $s \geq t$ we have $Z_{s}(x)=1$, so that $\tilde{Z}_{s} \neq \tilde{Z}_{\ell_{0}}$. This contradicts that $z(s)=\ell_{0}$ for infinitely many $s$. It follows that $\varphi_{e}^{0^{\prime \prime}}(0) \downarrow=g\left(\ell_{0}\right)$.

Suppose $\varphi_{e}^{0^{\prime \prime}}(0) \downarrow$, and let $t$ be the least true stage for which $\varphi_{e, t}^{Z_{t}}(0) \downarrow$ and $\tilde{Z}_{t} \subseteq Z$. It follows that for any true stage $s \geq t$ we must have $\tilde{Z}_{t}=\tilde{Z}_{s}$, and of course $K_{t} \subseteq K_{s}$, so that $z(s)=z(t)$. Therefore, if $\varphi_{e}^{0^{\prime \prime}}(0) \downarrow$, then there is some $\ell$ with $z(s)=\ell$ for infinitely many $s$ (namely $\ell=z(t)$ ), and $g(\ell)=\varphi_{e}^{0^{\prime \prime}}(0)$.

Finally, we show that $z$ satisfies the condition stated in Remark 5.2 , that $z(s)<$ $\ell \leq s$ implies that $z(t) \neq \ell$ for every $t \geq s$. Since $z(s)<s, \varphi_{e, s}^{Z_{z(s)}}(0) \downarrow$. If $K_{\ell} \nsubseteq K_{s}$, then $K_{\ell} \nsubseteq K_{t}$ for any $t \geq s$, so that $z(t) \neq \ell$ for all $t \geq s$. If $K_{\ell} \subseteq K_{s}$, then $K_{z(s)} \subseteq K_{\ell} \subseteq K_{s}$ and $\tilde{K}_{z(s)}=\tilde{K}_{\ell}=\tilde{K}_{s}$, so that $z(\ell)=z(s)<\ell$. Since for every $t$, $z(z(t))=z(t)$, we can never have $z(t)=\ell$.

5.2. Infinite sums of Boolean algebras. The second tool is $\omega$-sums of Boolean algebras. Given a sequence of Boolean algebras $\left\{\mathcal{A}_{i}\right\}_{i \in \omega}$, we define

$$
\mathcal{A}=\sum_{i \in \omega} \mathcal{A}_{i}
$$


to be the Boolean algebra whose domain is the set of infinite sequences which are eventually constant and equal to either zero or one. In other words, the domain of $\mathcal{A}$ is

$$
\mathcal{A}=\left\{\left\langle a_{i}: i \in \omega\right\rangle: a_{i} \in \mathcal{A}_{i},\left(\exists n \forall k>n\left(a_{k}=0_{\mathcal{A}_{k}}\right)\right) \vee\left(\exists n \forall k>n\left(a_{k}=1_{\mathcal{A}_{k}}\right)\right)\right\},
$$

and the operations are calculated coordinatewise. (See [Mon89, Ch. 4, §11] for more information on this operation, that they call the free product.)

Lemma 5.3. Let $\left\{\mathcal{A}_{i}\right\}_{i \in \omega}$ be a $Z$-computable sequence of uniformly $n$-Z-approximable Boolean algebras. Let $\gamma$ be a non-exclusive $n$-indecomposable type, and suppose that each $\mathcal{A}_{i}$ has type $\gamma$. Then $\sum_{i \in \omega} \mathcal{A}_{i}$ is also $n$-Z-approximable and has $n$-indecomposable type $\gamma$.

Proof. For each $i \in \omega$, let $\left\{\mathcal{A}_{i}[s]\right\}_{s \in \omega}$ be an $n$-Z-approximation of $\mathcal{A}_{i}$. For notational convenience, assume that for every $i, \mathcal{A}_{i}[0]$ is the two-element Boolean algebra and $\mathfrak{t}_{n}^{\mathcal{A}_{i}[0]}\left(1_{\mathcal{A}_{i}}\right)=\gamma$. Define $\mathcal{A}[s] \subseteq \mathcal{A}$ as follows:

$\mathcal{A}[s]=\left\{\left\langle a_{0}, a_{1}, \ldots\right\rangle: \forall i \leq s a_{i} \in \mathcal{A}_{i}[s] \&\left(\left(\forall i>s a_{i}=0_{\mathcal{A}_{i}}\right) \vee\left(\forall i>s a_{i}=1_{\mathcal{A}_{i}}\right)\right)\right\}$.

In other words,

$$
\mathcal{A}[s] \cong \mathcal{A}_{0}[s] \oplus \mathcal{A}_{1}[s] \oplus \cdots \oplus \mathcal{A}_{s}[s] \oplus \mathcal{A}_{s+1}[0] .
$$

Let $\mathfrak{t}_{n}^{s}\left(\left\langle a_{0}, a_{1}, \ldots\right\rangle\right)=\sum_{i \leq s} \mathfrak{t}_{n}^{\mathcal{A}_{i}[s]}\left(a_{i}\right)+\mathfrak{t}_{n}^{\mathcal{A}_{s+1}[s]}\left(a_{s+1}\right)$. Suppose that $\left\langle a_{0}, a_{1}, \ldots\right\rangle \in$ $\mathcal{A}[s-1]$. Then, $\mathfrak{t}_{n}^{\mathcal{A}_{i}[s-1]}\left(a_{i}\right) \leq_{n} \mathfrak{t}_{n}^{\mathcal{A}_{i}[s]}\left(a_{i}\right)$ for each $i<s$ and $\mathfrak{t}_{n}^{\mathcal{A}_{s}[s-1]}\left(a_{s}\right)=$ $\mathfrak{t}_{n}^{\mathcal{A}_{s}[s]}\left(a_{s}\right)+\mathfrak{t}_{n}^{\mathcal{A}_{s+1}[s]}\left(a_{s+1}\right)$, because all terms are either 0 or $\gamma$ (by assumption, $\gamma+\gamma=$ $\gamma)$. It then follows that $\mathfrak{t}_{n}^{s-1}\left(\left\langle a_{0}, a_{1}, \ldots\right\rangle\right) \leq_{n} \mathfrak{t}_{n}^{s}\left(\left\langle a_{0}, a_{1}, \ldots\right\rangle\right)$ by [HM, Lemma 7.8]. Thus, $\mathcal{A}[s]$ is a finite $n$-labeled Boolean algebra and $\mathcal{A}[s-1] \leq_{n} \mathcal{A}[s]$. To see that $\{\mathcal{A}[s]\}_{s \in \omega}$ is an $n$-Z-approximation, we have to show that every minimal element $a$ of $\mathcal{A}[s-1]$ splits in $\mathcal{A}[s]$ into a $\mathfrak{t}_{n}^{s}(a)$-full partition. Observe that if $a=\left\langle a_{0}, a_{1}, \ldots\right\rangle$ is a minimal element of $\mathcal{A}[s-1]$, then either, for some $i<s, a_{i}$ is a minimal nonzero element of $\mathcal{A}_{i}[s-1]$ and $a_{j}=0$ for $j \neq i$, or $a_{i}=0$ for $i<s$ and $a_{i}=1_{\mathcal{A}_{i}}$ for $i \geq s$. In the former case, we have that $a_{i}$ splits in $\mathcal{A}_{i}[s]$ into a $\mathfrak{t}_{n}^{\mathcal{A}_{i}[s]}\left(a_{i}\right)$-full partition. In the latter case, we have that $1_{\mathcal{A}_{s}}$ splits in $\mathcal{A}_{s}[s]$ into a $\gamma$-full partition. In either case, $a$ splits in $\mathcal{A}[s]$ into a $\mathfrak{t}_{n}^{s}(a)$-full partition.

Finally, since $\gamma$ is a non-exclusive $n$-indecomposable type, $\gamma=\gamma+\gamma$ follows from Lemma 2.20, and so, $\mathfrak{t}_{n}^{s}\left(\left\langle 1_{\mathcal{A}_{0}}, 1_{\mathcal{A}_{1}}, \ldots\right\rangle\right)=\gamma+\ldots+\gamma=\gamma$ for all $s$.

5.3. Listing of all $n$ - $Z$-approximable. In this subsection we show that there is a $0^{\prime}$-computable enumeration of $(n+1)$ - 0 '-approximations which lists all the Boolean algebras that have a computable $n$-approximation.

Lemma 5.4. Suppose $s>0$ and $\left\{\left(\mathcal{A}_{k}, \mathfrak{t}_{n}^{k}\right)\right\}_{k<s}$ is a sequence of finite n-labeled Boolean algebras that satisfy the conditions of Definition 3.4. Then there is an $n$-approximation $\left\{\left(\mathcal{A}_{k}, \mathfrak{t}_{n}^{k}\right)\right\}_{k \in \omega}$ extending $\left\{\left(\mathcal{A}_{k}, \mathfrak{t}_{n}^{k}\right)\right\}_{k<s}$.

Proof. We show how to build $\left(\mathcal{A}_{s}, \mathfrak{t}_{n}^{s}\right)$. The rest of the sequence is then built in the same way, one step at a time. For each $a \in \mathcal{A}_{s-1}$, let $\mathfrak{t}_{n}^{s}(a)=\mathfrak{t}_{n}^{s-1}(a)$. What we need to do now is to build a full partition for each minimal element of $\mathcal{A}_{s-1}$. Now, for each minimal $a \in \mathcal{A}_{s-1}$, we add new disjoint elements $\left\{a_{\gamma}: \gamma \in \mathfrak{t}_{n}^{s}(a)\right\} \cup\{\hat{a}\}$ below $a$, let $\mathfrak{t}_{n}^{s}\left(a_{\gamma}\right)$ be such that $\left(\mathfrak{t}_{n}^{s}\left(a_{\gamma}\right)\right)_{n-1}=\gamma$ and $\mathfrak{t}_{n}^{s}(a) \triangleleft_{n} \mathfrak{t}_{n}^{s}\left(a_{\gamma}\right)$, and let $\mathfrak{t}_{n}^{s}(\hat{a})=\mathfrak{t}_{n}^{s}(a)$. 
It then follows by Lemma 2.20 that

$$
\mathfrak{t}_{n}^{s}(a)=\mathfrak{t}_{n}^{s}(\hat{a})+\sum_{\gamma \in \mathfrak{t}_{n}^{s}(a)} \mathfrak{t}_{n}^{s}\left(a_{\gamma}\right) .
$$

This is a $\mathfrak{t}_{n}^{s}(a)$-full partition of $a$, so that once we have established the existence of these $\mathfrak{t}_{n}^{s}\left(a_{\gamma}\right) \in \mathbf{B F}_{n}$, we can conclude that the sequence $\left\{\left(\mathcal{A}_{k}, \mathfrak{t}_{n}^{k}\right)\right\}_{k<s+1}$ also satisfies the conditions of Definition 3.4 .

It remains to show that for each $\alpha \in \mathbf{B F}_{n}$ and each $\gamma \in \alpha$, there is a $\gamma^{*} \in \mathbf{B F}_{n}$ with $\left(\gamma^{*}\right)_{n-1}=\gamma$ and $\alpha \triangleleft_{n} \gamma^{*}$. This is derived from the following fact. Let $\mathcal{B}$ be a Boolean algebra which is $n$-indecomposable with $n$-bf-type $\alpha$ and $\gamma \in \alpha$. By Definition 2.6 there is a partition $1_{\mathcal{B}}=b_{0} \dot{\vee} b_{1}$ such that $b_{1}$ is an $(n-1)$ indecomposable subalgebra with $t_{n-1}\left(b_{1}\right)=\gamma$. Furthermore, by Theorem 2.5)(b), there is a partition $b_{1}=c_{0} \dot{V} \ldots \dot{V} c_{k}$ into $n$-indecomposable subalgebras such that $t_{n-1}\left(c_{0}\right)=\gamma$. Since $\operatorname{dc} t_{n}\left(c_{0}\right) \subseteq \operatorname{dc} t_{n}(\mathcal{B})=\operatorname{dc} \alpha$ (see the last paragraph of Remark 2.14), it follows by Definition 2.18 that $\alpha \triangleleft_{n} t_{n}\left(c_{0}\right)$.

Lemma 5.5. There is a $0^{\prime}$-computable listing $\left\{\mathcal{C}_{e}: e \in \omega\right\}$ of $(n+1)-0^{\prime}$-approximations such that each $n-0$ approximable Boolean algebra has an $(n+1)-0^{\prime}$-approximation on this list.

Proof. Let $\left\{\varphi_{e}\right\}_{e \in \omega}$ be a list of the 0-computable partial functions. If $\left\{\left(\mathcal{D}_{s}, \mathfrak{t}_{n}^{s}\right)\right\}_{s \in \omega}$ is an $n$-0-approximation of $\mathcal{D}$, then for some $e, \varphi_{e}$ generates this approximation, meaning that $\varphi_{e}(s)=\left(\mathcal{D}_{s}, \mathfrak{t}_{n}^{s}\right)$. For each $e$, we will use $0^{\prime}$ to construct an $(n+1)$ approximation $\mathcal{C}_{e}=\left\{\left(\mathcal{C}_{e, s}, \mathfrak{t}_{n+1}^{s}\right)\right\}_{s \in \omega}$ so that if $\varphi_{e}$ generates an $n$-0-approximation of $\mathcal{D}$, then $\mathcal{C}_{e}$ will be an $(n+1)$-approximation for $\mathcal{D}$; but, if $\varphi_{e}$ does not generate an $n$-0-approximation, then $\mathcal{C}_{e}$ will still be an $(n+1)$-approximation of some algebra.

Fix $\varphi_{e}$, a purported $n$-0-approximation, and let $\left(\mathcal{D}_{s}, \mathfrak{t}_{n}^{s}\right)=\varphi_{e}(s)$ for each $s \in \omega$ where $\varphi_{e}(s) \downarrow$. Given $s$, if $\varphi_{e}(k)$ is defined for all $k<s$, then it is computable to check that the conditions of Definition 3.4 are satisfied for $\left\{\left(\mathcal{D}_{k}, \mathfrak{t}_{n}^{k}\right)\right\}_{k<s}$.

Using $0^{\prime}$ we can check that the following condition is met: For every $t$ and each stage $u$, if for each $k<t, \varphi_{e}(k)$ converges by stage $u$, then $\left\{\left(\mathcal{D}_{k}, \mathfrak{t}_{n}^{k}\right)\right\}_{k<t}$ meets the conditions of Definition 3.4. This is a $\Pi_{1}^{0}$ condition, so it is computable in $0^{\prime}$. If the condition does not hold, then $\varphi_{e}$ does not give an $n$-approximation, and hence we need not worry about how to build $\left\{\left(\mathcal{C}_{e, s}, \mathfrak{t}_{n+1}^{s}\right)\right\}_{s \in \omega}$, so we let it be any $(n+1)$-approximation. Suppose now that this condition holds.

The problem for the construction is that $0^{\prime}$ cannot check whether $\varphi_{e}$ is total. So, we will verify this one step at a time; while $\varphi_{e}$ looks total, we define the $(n+1)$ approximation $\left\{\left(\mathcal{C}_{e, s}, \mathfrak{t}_{n+1}^{s}\right)\right\}_{s \in \omega}$ by stages. If we ever discover that $\varphi_{e}$ does not converge on some input $s$, then we continue the construction of $\left\{\left(\mathcal{C}_{e, k}, \mathfrak{t}_{n+1}^{k}\right)\right\}_{k<s}$ using the previous lemma. We now carry out this construction.

We first show how $0^{\prime}$ can use $\left\{\left(\mathcal{D}_{s}, \mathfrak{t}_{n}^{s}\right)\right\}_{s \in \omega}$ to compute predicates $\mathrm{R}_{\alpha}^{e}$ (for $\alpha \in$ $\left.\mathbf{B F}_{n+1}\right)$, which will be the $(n+1)$-back-and-forth predicates $\mathrm{R}_{\alpha}$ on $\mathcal{D}$, provided $\left\{\left(\mathcal{D}_{s}, \mathfrak{t}_{n}^{s}\right)\right\}_{s \in \omega}$ is truly an $n$-approximation for $\mathcal{D}$. For each $\alpha \in \mathbf{B F}_{n+1}$, let $\bar{I}_{\alpha}$ be the set of sequences $\left\{\beta_{0}, \ldots, \beta_{k}\right\} \in \mathbf{B F}_{n}^{<\omega}$ for which there are $\alpha_{0}, \ldots, \alpha_{k} \in \mathbf{B F}_{n}$ such that for some $i \leq k, \alpha_{i}=(\alpha)_{n}$; for each $j \neq i, \alpha_{j} \in \alpha$; and for each $i \leq k$, $\beta_{i} \leq_{n} \alpha_{i}$. Note that $\bar{I}_{\alpha}$ is computable because there are only finitely many possible ways of selecting $\alpha_{0}, \ldots, \alpha_{k}$ from $\alpha \cup\left\{(\alpha)_{n}\right\}$. We will show the following for any 
Boolean algebra $\mathcal{B}$ and $b \in \mathcal{B}$ :

$$
\begin{aligned}
\mathrm{R}_{\alpha}(b) \Longleftrightarrow \forall b_{0} \dot{\vee} \ldots \dot{\vee} b_{k}=b \\
\quad\left[(\forall i=0, \ldots, k) T_{n}^{\mathcal{B}}\left(b_{i}\right) \in \mathbf{B F}_{n} \Longrightarrow\left\{T_{n}^{\mathcal{B}}\left(b_{0}\right), \ldots, T_{n}^{\mathcal{B}}\left(b_{k}\right)\right\} \in \bar{I}_{\alpha}\right]
\end{aligned}
$$

Recall that there is an $(n+1)$-indecomposable Boolean algebra $\mathcal{A}_{\alpha}$ with $T_{n}\left(\mathcal{A}_{\alpha}\right)=\alpha$ and that we defined $\mathrm{R}_{\alpha}(b)$ to be equivalent to $\mathcal{A}_{\alpha} \leq_{n+1} \mathcal{B}\left\lceil b\right.$. Suppose $\mathrm{R}_{\alpha}(b)$ holds and let $b=b_{0} \dot{\vee} \ldots \dot{\vee} b_{k}$ be a partition with $T_{n}^{\mathcal{B}}\left(b_{i}\right) \in \mathbf{B F}_{n}$ for each $i \leq k$. Then by Lemma 2.24 there is a partition $1_{\mathcal{A}}=a_{0} \dot{\mathrm{V}} \ldots \dot{\mathrm{V}} a_{k}$ with $T_{n}^{\mathcal{B}}\left(b_{i}\right) \leq_{n} T_{n}^{\mathcal{A}_{\alpha}}\left(a_{i}\right)$ for all $i \leq k, T_{n}^{\mathcal{A}_{\alpha}}\left(a_{i}\right)=(\alpha)_{n}$ for some $j \leq k$, and $T_{n}^{\mathcal{A}_{\alpha}}\left(a_{i}\right) \in \alpha$ for each $i \neq j$. This establishes that $\left\{T_{n}^{\mathcal{B}}\left(b_{0}\right), \ldots, T_{n}^{\mathcal{B}}\left(b_{k}\right)\right\} \in \bar{I}_{\alpha}$. Conversely, suppose the right side of the equivalence holds. We will show that $\mathcal{A}_{\alpha} \leq_{n+1} \mathcal{B}\left\lceil b\right.$. Let $b=b_{0} \dot{V} \ldots \dot{\vee} b_{k}$ be a partition with $T_{n}^{\mathcal{B}}\left(b_{i}\right) \in \mathbf{B F}_{n}$ and $\left\{T_{n}^{\mathcal{B}}\left(b_{0}\right), \ldots, T_{n}^{\mathcal{B}}\left(b_{k}\right)\right\} \in \bar{I}_{\alpha}$. Then there are $\alpha_{0}, \ldots, \alpha_{k}$ with $T_{n}^{\mathcal{B}}\left(b_{i}\right) \leq_{n} \alpha_{i}$ for each $i \leq k, \alpha_{j}=(\alpha)_{n}$ for some $j \leq k$, and $\alpha_{i} \in \alpha$ for all $i \neq j$. By Lemma 2.23 there is a partition $1_{\mathcal{A}_{\alpha}}=a_{0} \dot{V} \ldots \dot{V} a_{k}$ with $T_{n}^{\mathcal{A}_{\alpha}}\left(a_{i}\right)=\alpha_{i}$ for each $i \leq k$, and so $T_{n}^{\mathcal{B}}\left(b_{i}\right) \leq_{n} T_{n}^{\mathcal{A}_{\alpha}}\left(a_{i}\right)$. Thus, $\mathcal{A}_{\alpha} \leq_{n+1} \mathcal{B}\lceil b$.

The above equivalence of $\mathrm{R}_{\alpha}$ justifies the following definition of the predicates $\mathrm{R}_{\alpha}^{e}\left(\right.$ for $\left.\alpha \in \mathbf{B F}_{n+1}\right)$. For each $a \in \bigcup_{s} \mathcal{D}_{s}$, let $s$ be the least stage with $a \in \mathcal{D}_{s}$; then

$\left(^{*}\right) \mathrm{R}_{\alpha}^{e}(a)$ if and only if for all $\ell \geq s$ and all stages $u$, if $\varphi_{e}(\ell)=\left(\mathcal{D}_{\ell}, \mathfrak{t}_{n}^{\ell}\right)$ converges by stage $u$, then for all partitions $a=a_{0} \dot{V} \ldots \dot{V} a_{k}$ with $a_{0}, \ldots, a_{k} \in$ $\mathcal{D}_{\ell}$ and $(\forall i=0, \ldots, k) \mathfrak{t}_{n}^{\mathcal{D}_{\ell}}\left(a_{i}\right) \in \mathbf{B F}_{n}$, we have that $\left\{\mathfrak{t}_{n}^{\ell}\left(a_{0}\right), \ldots, \mathfrak{t}_{n}^{\ell}\left(a_{k}\right)\right\} \in$ $\bar{I}_{\alpha}$.

Note that $(*)$ is a $\Pi_{1}^{0}$ condition and so is decidable by $0^{\prime}$, but only when $\left(\mathcal{D}_{s}, t_{n}^{s}\right)$ is defined for all $s \in \omega$ will these predicates reflect possible back-and-forth relations. Just as in the proof of Theorem [3.7, let $u$ be a $0^{\prime}$-computable partial function on the domain of $\mathcal{D}$ defined so that, for each $a \in \mathcal{D}, u(a)$ is the $\geq_{n+1}$-greatest $(n+1)$-bf-type $\gamma \in \mathbf{B F}_{n+1}$ such that $\mathrm{R}_{\gamma}^{e}(a)$ holds, if such a $\gamma$ exists, and undefined otherwise.

We will construct an $(n+1)-0^{\prime}$-approximable Boolean algebra $\mathcal{C}_{e}$ (as in Definition 3.4) using these relations $\mathrm{R}_{\alpha}^{e}$ exactly as in Theorem 3.7 while enumerating $\left(\mathcal{D}_{s}, \mathfrak{t}_{n}^{s}\right)$. The construction builds an $(n+1)$-approximation $\left\{\left(\mathcal{C}_{e, s}, \mathfrak{t}_{n+1}^{s}\right)\right\}_{s \in \omega}$ in stages $s$ using a $0^{\prime}$ oracle. At stage $s$ we will build a finite $(n+1)$-labeled algebra $\left(\mathcal{C}_{e, s}, \mathfrak{t}_{n+1}^{s}\right)$ and then let $\mathcal{C}_{e}=\bigcup_{s} \mathcal{C}_{e, s}$. We now drop the subscript $e$ and write just $\mathcal{C}$ for $\mathcal{C}_{e}$.

To begin, if $\left\{\left(\mathcal{D}_{s}, \mathfrak{t}_{n}^{s}\right)\right\}_{s \in \omega}$ fails to be an $n$-approximation, we can choose any $(n+1)$-approximation $\left\{\left(\mathcal{C}_{s}, t_{n+1}^{s}\right)\right\}_{s \in \omega}$. So, we may assume that if $\varphi_{e}$ is total, then $\left(\mathcal{D}_{s}, \mathfrak{t}_{n}^{s}\right)_{s \in \omega}$ really is an $n$-approximation of a Boolean algebra $\mathcal{D}$. Let

$$
\mathcal{D}=\bigcup_{s}\left\{\mathcal{D}_{s}:(\forall t \leq s) \phi_{e}(s) \downarrow\right\}
$$

and let $\left\{d_{0}=1_{\mathcal{D}}, d_{1}, d_{2}, \ldots\right\}$ be a $0^{\prime}$ enumeration of $\mathcal{D}$. There are two reasons $\mathcal{D}$ could be finite: $\left(\mathcal{D}_{s}, t_{n}^{s}\right)_{s \in \omega}$ is an $n$-approximation of a finite Boolean algebra or $\varphi_{e}$ is not total. In the first case there is a stage $s$ in which every minimal element of $\mathcal{D}_{s}$ is an atom of $\mathcal{D}$, and in the second case there is a stage $s$ at which $\phi_{e}(s) \uparrow$, so in either case $0^{\prime}$ can decide if $\mathcal{D}$ is finite by this stage $s$.

Suppose that we have already constructed a sequence of finite $(n+1)$-labeled algebras $\left\{\left(\mathcal{C}_{k}, \mathfrak{t}_{n+1}^{k}\right)\right\}_{k<s}$ which satisfy the conditions of Definition 3.4 and $\left\{d_{0}, \ldots, d_{s-1}\right\}$ $\subseteq \mathcal{C}_{s-1}$, but that $0^{\prime}$ has not yet determined if $\mathcal{D}$ is finite. If at any point in the 
current stage of the construction $0^{\prime}$ determines that $\phi_{e}$ is not total, then the construction will continue by applying Lemma 5.4 to $\left\{\left(\mathcal{C}_{k}, \mathfrak{t}_{n+1}^{k}\right)\right\}_{k<s}$ (or choose any $(n+1)$-approximation if $s=0)$. The remainder of the construction shows how to extend $\left\{\left(\mathcal{C}_{k}, \mathfrak{t}_{n+1}^{k}\right)\right\}_{k<s}$ to $\left\{\left(\mathcal{C}_{k}, \mathfrak{t}_{n+1}^{k}\right)\right\}_{k<s+1}$ so that this new sequence also satisfies the conditions of Definition 3.4 and $d_{s} \in \mathcal{C}_{s}$.

We will add $d_{s}$ to $\mathcal{C}_{s-1}$ as follows: for each minimal element $a$ in $\mathcal{C}_{s-1}$ (or $a=1_{\mathcal{D}}$ if $s=0$ ), look for a partition $a_{0}, \ldots, a_{\ell} \in \mathcal{D}$ of $a$ such that

- $u\left(a_{i}\right)$ is defined for each $i \leq \ell$,

- for some $h \leq \ell, a \wedge d_{s}=\bigvee_{i=0}^{h} a_{i}$ and $a-d_{s}=\bigvee_{i=h+1}^{\ell} a_{i}$,

- $\mathfrak{t}_{n+1}^{s-1}(a) \leq_{n+1} \sum_{i=0}^{\ell} u\left(a_{i}\right)$ (this condition is ignored if $s=0$ since $\mathfrak{t}_{n+1}^{s-1}\left(1_{\mathcal{D}}\right)$ is undefined),

- the elements $a_{0}, \ldots, a_{\ell}$ form a $\sum_{i=0}^{\ell} u\left(a_{i}\right)$-full partition of $a$.

We also enumerate $\varphi_{e}$ while looking for such a partition of $a$. If $\varphi_{e}$ is total, then there necessarily exists such a partition of $a$ (applying Remark 3.3), so that if no such partition of $a$ exists, then $\varphi_{e}$ is not total and $0^{\prime}$ will discover this fact at the current stage.

Let $\mathcal{C}_{s}$ be the extension of $\mathcal{C}_{s-1}$ generated by the partitions $a_{0}, \ldots, a_{\ell}$ of the minimal elements $a$ in $\mathcal{C}_{s-1}$. For each of these new elements $a_{i}$, let $\mathfrak{t}_{n+1}^{s}\left(a_{i}\right)=u\left(a_{i}\right)$. For each $b \in \mathcal{C}_{s}$ with $b=\bigvee_{i=0}^{j} c_{i}$, where the $c_{i}$ 's are minimal elements in $\mathcal{C}_{s}$, let $\mathfrak{t}_{n+1}^{s}(b)=\sum_{i=0}^{j} u\left(c_{i}\right)$. Just as in the proof of Theorem 3.7, $\left\{\left(\mathcal{C}_{k}, \mathfrak{t}_{n+1}^{k}\right)\right\}_{k<s+1}$ satisfies the conditions of Definition 3.4. If $0^{\prime}$ decides that $\mathcal{D}$ is finite because all minimal elements in $\mathcal{C}_{s}$ are labeled atom, then $\left\{\left(\mathcal{C}_{k}, \mathfrak{t}_{n+1}^{k}\right)\right\}_{k<s+1}$ is an $(n+1)$ approximation of $\mathcal{D}$ and the construction can terminate. This completes stage $s+1$ of the construction.

\section{NO ZERO-TRIPLE PROOF}

The following theorem is the heart of our construction of a low 5 Boolean algebra which is not isomorphic to a computable Boolean algebra via a $0^{(7)}$-computable isomorphism. We will use the relativization of this theorem to $0^{(4)}$ in the proof of Theorem 6.8

Theorem 6.1. There exists a 5-0'-approximable Boolean algebra which is not $0^{\prime \prime \prime}$ isomorphic to any 4-0-approximable Boolean algebra.

Proof. Let $\left\{\mathcal{C}_{i}: i \in \omega\right\}$ be a $0^{\prime}$-computable listing of 5 - $0^{\prime}$-approximations to Boolean algebras which includes all 4-0-approximable Boolean algebras, as determined by Lemma 5.5. We assume that the listing is such that there is a $0^{\prime}$-computable function $h: \omega \times \omega \rightarrow \omega$ with the property that for each $c \in \mathcal{C}_{i}$ we have

$$
\mathcal{C}_{h(i, c)}=\mathcal{C}_{i} \uparrow c
$$

and if $c \notin C_{i}$, then $\mathcal{C}_{h(i, c)}$ is the trivial Boolean algebra. Let $\left\{\Phi_{e}^{X}: e \in \omega\right\}$ be a computable listing of Turing functionals.

We are going to build a 5-0'-approximable Boolean algebra

$$
\mathcal{A}=\sum_{e, i \in \omega} \mathcal{A}_{\langle e, i\rangle},
$$

that, for each $e, i \in \omega$, satisfies the requirement:

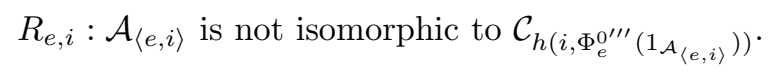


Satisfying these requirements suffices to establish the theorem. Suppose, toward a contradiction, that $\Phi_{e}^{0^{\prime \prime \prime}}: \mathcal{A} \rightarrow \mathcal{C}_{i}$ is an isomorphism; then we must have $\mathcal{A}_{\langle e, i\rangle}$ isomorphic to $\left.\left.\mathcal{C}_{i} \uparrow \Phi_{e}^{0^{\prime \prime \prime}}\left(1_{\mathcal{A}_{\langle e, i\rangle}}\right)=\mathcal{C}_{h\left(i, \Phi_{e}^{0^{\prime \prime \prime \prime}}\left(1_{\mathcal{A}}\langle e, i\rangle\right.\right.}\right)\right)$, contradicting $R_{e, i}$.

Fix $e$ and $i$. By the uniformity of a relativization of Lemma 5.1, we can compute indices for two $0^{\prime}$-computable functions

$$
z_{e, i}: \omega \rightarrow \omega \quad \text { and } \quad g_{e, i}: \omega \rightarrow \omega
$$

such that

$$
g_{e, i}\left(\ell_{0}\right)=\Phi_{e}^{0^{\prime \prime \prime}}\left(1_{\mathcal{A}_{\langle e, i\rangle}}\right),
$$

where $\ell_{0} \in \omega$ is the only number satisfying $z_{e, i}(s)=\ell_{0}$ for infinitely many $s$. If $\Phi_{e}^{0^{\prime \prime \prime}}\left(1_{\mathcal{A}_{\langle e, i\rangle}}\right) \uparrow$, then no $\ell$ occurs infinitely often.

The remainder of the proof is the construction of the algebra $\mathcal{A}_{\langle e, i\rangle}$ using the two $0^{\prime}$-computable functions $z_{e, i}$ and $g_{e, i}$ to guess the restricted algebra $\mathcal{C}_{h\left(i, \Phi_{e}^{0^{\prime \prime \prime}}\left(1_{\mathcal{A}_{\langle e, i\rangle}}\right)\right)}$. (If $\mathcal{C}_{i}$ is not the image of $\Phi_{e}^{0^{\prime \prime \prime}}$, then the construction is moot.) We will drop our reference to the subscripts $e$ and $i$ in what follows and just write $z, g, \mathcal{A}$, and $\mathcal{C}$. We abbreviate $\mathcal{C}_{h(i, g(\ell))}$ as $\mathcal{B}_{\ell}$. For simplicity, we will also drop the $0^{\prime}$ oracle, as we can relativize the proof later. The next proposition summarizes what we will show.

Proposition 6.2. Let $\left\{\mathcal{B}_{\ell}: \ell \in \omega\right\}$ be a computable sequence of 5-0-approximable Boolean algebras, and let $z: \omega \rightarrow \omega$ be a total computable function with the properties of the one in Lemma 5.1. We can build, uniformly in $z$ and $\left\{\mathcal{B}_{\ell}: \ell \in \omega\right\}$, a 5-0-approximable Boolean algebra $\mathcal{A}$ such that: if there is a number $\ell_{0}$ with the property that $z(s)=\ell_{0}$ for infinitely many $s$, then $\mathcal{A}$ is not isomorphic to $\mathcal{B}_{\ell_{0}}$.

We will build $\mathcal{A}$ as an $\omega$-sum (see Section 5.2) of a sequence of uniformly 5-0approximable Boolean algebras $\mathcal{A}_{\ell}$,

$$
\mathcal{A}=\sum_{\ell \in \omega} \mathcal{A}_{\ell}
$$

where $\mathcal{A}_{\ell}$ is a subalgebra we build on the guess that $\ell=\ell_{0}$.

We construct each $\mathcal{A}_{\ell}$ to be 5-0-approximable in stages $s$, so that $\left\{\mathcal{A}_{\ell}[s]: s \in \omega\right\}$ is computable uniformly in $\ell$. These approximations will all satisfy properties (D1)(D4) from Section 4 so that each $\mathcal{A}_{\ell}$ will have 5 -indecomposable type $w^{\infty}$; it will then follow from Lemma 5.3 that $\mathcal{A}$ is 5 -0-approximable with 5 -indecomposable type $w^{\infty}$. Let $\ell_{0}$ be the unique value (if it exists) such that for infinitely many $s$, $z(s)=\ell_{0}$. (If $\ell_{0}$ does not exist, think of $\ell_{0}$ as $\infty$.)

(B1) If $\ell<\ell_{0}$, then $\operatorname{num}\left(w^{\infty}, \mathcal{A}_{\ell}\right)=\infty$ and both $\operatorname{num}\left(w, \mathcal{A}_{\ell}\right), \operatorname{num}\left(u, \mathcal{A}_{\ell}\right)<\infty$.

(B2) If $\ell=\ell_{0}$, then either $\operatorname{num}\left(w^{\infty}, \mathcal{A}_{\ell}\right)=1$ and $\operatorname{num}\left(w, \mathcal{A}_{\ell}\right)=\infty$, or num $\left(w^{\infty}, \mathcal{A}_{\ell}\right)$ $=\infty$ and $\operatorname{num}\left(w, \mathcal{A}_{\ell}\right)<\infty$. In either case $\operatorname{num}\left(u, \mathcal{A}_{\ell}\right)=0$.

(B3) If $\ell_{0}<\ell$, then $\operatorname{num}\left(w^{\infty}, \mathcal{A}_{\ell}\right)=\infty, \operatorname{num}\left(w, \mathcal{A}_{\ell}\right)=0$ and $1<\operatorname{num}\left(u, \mathcal{A}_{\ell}\right)<$ $\infty$.

In the table below we list $\operatorname{num}\left(\gamma, \mathcal{A}_{\ell}\right)$ for $\gamma=u, w, w^{\infty}$. The symbol $\mathrm{f}$ means "finitely many".

\begin{tabular}{c||c|c|c} 
& $u$ & $w$ & $w^{\infty}$ \\
\hline \hline$\ell<\ell_{0}$ & $\mathrm{f}$ & $\mathrm{f}$ & $\infty$ \\
\hline$\ell=\ell_{0}$ & 0 & $\infty$ & 1 \\
\cline { 3 - 4 } & & $\mathrm{f}$ & $\infty$ \\
\hline$\ell>\ell_{0}$ & $\mathrm{f}$ & 0 & $\infty$
\end{tabular}


Thus, when $\ell_{0}<\infty, \mathcal{A}$ will contain either exactly one 1-w-atom (and infinitely many disjoint 5 -indecomposable elements of type $w$ ) or no 1-w-atom and only finitely many disjoint 5 -indecomposable elements of type $w$. In either case $\mathcal{A}$ will contain infinitely many disjoint 5 -indecomposable elements of type $u$ (at least one in each algebra $\mathcal{A}_{\ell}$ when $\ell>\ell_{0}$ ) and infinitely many of type $w^{\infty}$.

We will assign a worker $G_{\ell}$ to construct the algebra $\mathcal{A}_{\ell}$ using a strategy which will ensure that the following condition is met:

$$
G_{\ell}:\left(\exists^{\infty} s\right) z(s)=\ell \Longrightarrow \mathcal{A} \text { is not isomorphic to } \mathcal{B}_{\ell} .
$$

Of course, only $G_{\ell_{0}}$ has a real responsibility.

The goal of $G_{\ell}$ is to achieve one of the following conditions:

$(\mathrm{S} 1) \operatorname{num}(w, \mathcal{A})<\operatorname{num}\left(w, \mathcal{B}_{\ell}\right)$.

(S2) $\mathcal{A}_{\ell}$ is a 1 - $w$-atom and, for every $b \in \mathcal{B}_{\ell}, \mathcal{B}_{\ell} \uparrow b$ is not a 1 -w-atom because one of the following applies:

(a) $\mathfrak{t}_{5}^{\mathcal{B}_{\ell}}(b) \neq w^{\infty}$

(b) $\operatorname{num}(u, b) \geq 1$, or

(c) there is a partition $b=b_{0} \dot{\vee} b_{1}$ with $\operatorname{num}\left(e_{6}, b_{0}\right), \operatorname{num}\left(e_{6}, b_{1}\right)=\infty$.

We describe $G_{\ell}$ 's strategy. First, if $\mathcal{B}_{\ell}$ does not have 5 -bf-type $w^{\infty}$, then we have nothing to do, as $\mathcal{A}$ will have 5 -bf-type $w^{\infty}$. So suppose it does, and hence from some stage onward $1_{\mathcal{B}_{\ell}}$ has type $w^{\infty}$; this is the moment when we start working for $G_{\ell}$. We will attempt to satisfy (S1) as follows: If we see some $b \in \mathcal{B}_{\ell}$ such that $\mathcal{B}_{\ell}\lceil b$ has more $w$ elements than $\mathcal{A}$ has so far, then we will restrain the overall production of $w$ elements in $\mathcal{A}$. If all these $w$ elements below $b$ stay with 5 -bf-type $w$ forever, we will end up satisfying (S1). However, some of these elements may increase their 5-bf-type to $u$. If this happens, then $b$ satisfies (S2b), and we can take one step toward making $\mathcal{A}_{\ell}$ a 1 -w-atom. Taking this step implies increasing the 5-bf-type of all but one of the $w^{\infty}$ minimal elements in $\mathcal{A}_{\ell}[s]$ to $w$ in $\mathcal{A}_{\ell}[s+1]$. Notice that this may injure the restraint that other elements $b$ are imposing on the production of $w$ elements, so we will have to order the elements $b \in \mathcal{B}_{\ell}$ according to some order of priorities. We will argue that if we never manage to satisfy (S1), then we will satisfy (S2), as follows. For every $w^{\infty}$ element $b$ of $\mathcal{B}_{\ell}$, there are three possibilities: (i) $b$ splits into two or more $w^{\infty}$ elements, (ii) there is some $u$ element below $b$, and (iii) there are too many $w$ elements below $b$. Hence, we would win by $(\mathrm{S} 2 \mathrm{c})$, (S2b), or (S1), respectively. As long as there are no $u$ elements and too few $w$ elements below $b$, we believe (S2c) will hold. At stages where that occurs we will wait until a large number of $e_{6}$ elements appear in $\mathcal{B}_{\ell}\lceil b$. The reason is that if (S2c) does not actually hold, then all these $e_{6}$ elements will have to turn into either $u$ or $w$ elements. If we see any $u$ element below $b$, then $(\underline{S 2 b})$ holds. Otherwise, we will see a lot of $w$ elements at once - enough to act towards (S1), as mentioned above.

The algebra $\mathcal{A}$ will be constructed from the algebras $\mathcal{A}_{\ell}$ so that at stage $s$,

$$
\mathcal{A}[s] \cong \mathcal{A}_{0}[s] \oplus \mathcal{A}_{1}[s] \oplus \cdots \oplus \mathcal{A}_{s}[s] \oplus \mathcal{A}_{s+1}[0] .
$$

We write $\mathcal{A}_{\leq \ell}$ to denote $\sum_{i=0}^{\ell} \mathcal{A}_{i}$.

We now describe the construction of the sequence $\left\{\left(\mathcal{A}_{\ell}[s], \mathfrak{t}_{5}^{\ell[s]}\right): s \in \omega\right\}$, which will be computable uniformly in $\ell$. At stages $s \leq \ell$, no work to satisfy the requirement will be done; instead the algebra is extended at each stage using canonical partitions (from Section 44), starting with the two-element algebra whose top element will have 5-bf-type $w^{\infty}$. No elements of 5-bf-type $u$ or $v$ are in these early stages. 
At stages $s>\ell$, the construction extends $\mathcal{A}_{\ell}[s]$, working to satisfy our requirement, and ensuring that there is at least one minimal element of 5-indecomposable type $w^{\infty}$ and that all other minimal elements which have 4-indecomposable type $e_{6}$ will have 5 -indecomposable type $u, w$, or $w^{\infty}$. The sequence $\left\{\left(\mathcal{A}_{\ell}[s], \mathfrak{t}_{5}^{\ell[s]}\right): s \in \omega\right\}$ will also satisfy conditions (D1)-(D4) from Section 4 and will be a 5-approximation for the algebra $\mathcal{A}_{\ell}=\bigcup_{s} \mathcal{A}_{\ell}[s]$.

For each stage $s$, we will define $r_{\ell, s} \in \omega$, which will tell us how far to look into the approximation of $\mathcal{B}_{\ell}$. We will write $r_{s}$ when $\ell$ is clear from the context.

Construction of $\mathcal{A}_{\ell}$ :

Stage $s=0$. Let $A_{\ell}[0]$ be the Boolean algebra with two elements $\{0,1\}$, where $\mathfrak{t}_{5}^{\ell[0]}(1)=w^{\infty}$. Set $r_{0}=0$.

Stage $1 \leq s+1 \leq \ell$. Extend $\mathcal{A}_{\ell}[s]$ to $\mathcal{A}_{\ell}[s+1]$ by splitting each minimal element $a$ of $\mathcal{A}_{\ell}[s]$ into a canonical $\mathfrak{t}_{5}^{\ell[s+1]}(a)$-full partition as described in Section 4. Set $r_{s+1}=0$.

Stage $s+1>\ell$. There are two steps. In Step 1, the construction makes modifications only to the 5-bf-types of elements in $\mathcal{A}_{\ell}[s]$ (which will change only by increase, if at all). Step 2 is where $\mathcal{A}_{\ell}[s+1]$ is extended according to the canonical partitions discussed in Section 4.

Step 1. The worker $G_{\ell}$ is in one of three states: cancelled, inactive, or active.

$G_{\ell}$ is cancelled at stage $s+1$. This happens when $z(s)<\ell$ and $s$ is the first stage when $s>\ell$ and $z(s)<\ell$. By Remark 5.2, we will never have $z(s)=\ell$ after this stage, so we need not worry about $G_{\ell}$ anymore. If there are any minimal $w$ elements in $\mathcal{A}_{\ell}[s]$, increase their 5-bf-type to $u$ (and if there are no minimal elements of 5-bf-type $w$, then split an element of 5-bf-type $w^{\infty}$ to include a new minimal element of 5-bf-type $u$ ). Leave the 5-bf-types of all the other minimal elements unchanged. Define $r_{s+1}=r_{s}+1$ and proceed to Step 2 .

$G_{\ell}$ is inactive at stage $s+1$. This happens when either $\ell \neq z(s)$ or $G_{\ell}$ has been cancelled at some previous stage. No change is made to the values of $\mathfrak{t}_{5}^{\ell[s]}$. Define $r_{s+1}=r_{s}+1$ and proceed to Step 2 .

$G_{\ell}$ is active at stage $s+1$. This happens when $z(s)=\ell$. We will call $s+1$ an $\ell$-stage. There are two possible strategies for active $G_{\ell}$ : restrained or unrestrained.

- restrained: Restrain the production of $w$ elements at this stage because condition (S1) currently holds.

- unrestrained: It looks as though condition (S2) may hold at the end, so take one step toward building a 1-w-atom.

There are two phases: the strategy phase, where the strategy is determined, and the action phase, where any actions modifying the types of elements occur.

Strategy phase. The first step is to look far enough into the approximation of $\mathcal{B}_{\ell}$, or in other words, to define $r_{s+1}$ : For each minimal $e_{6}$ element $b \in \mathcal{B}_{\ell}\left[r_{s}\right]$, we search for a stage $r^{b} \geq r_{s}$ in the 5 -0-approximation of $\mathcal{B}_{\ell}$ at which one of the following conditions holds:

(W1) All minimal $e_{6}$ elements in $\mathcal{B}_{\ell}\left[r^{b}\right]\lceil b$ have exclusive 5 -indecomposable types.

(W2) $\operatorname{num}\left(e_{6}, \mathcal{B}_{\ell}\left[r^{b}\right]\lceil b) \geq 2 \cdot \operatorname{num}\left(e_{6}, \mathcal{A}[s]\right)+2\right.$ (i.e. there are many more $e_{6}$ elements in $\mathcal{B}_{\ell}\left[r^{b}\right]$ below $b$ than there are currently in $\left.\mathcal{A}[s]\right)$.

(W3) $\mathfrak{t}_{5}^{\mathcal{B}_{\ell}\left[r^{b}\right]}(1)>_{5} w^{\infty}$ or some minimal element $a \in \mathcal{B}_{\ell}\left[r^{b}\right]$ has 4 -bf-type not in $\left\{(\sigma)_{4}: \sigma \in D\right\}$. 
Once this task has been completed for each minimal element $b$ of $B_{\ell}\left[r_{s}\right]$, set $r_{s+1}$ to the maximum of all the $r^{b}$ and $r_{s}+1$.

If we find a stage at which (W3) holds, then we know that $\mathcal{A}$ cannot be isomorphic to $\mathcal{B}_{\ell}$, so we cancel $G_{\ell}$ at the first stage we discover (W3) to hold and proceed as described earlier. Suppose that (W3) is not found to hold during the current stage.

We say that $b \in B_{\ell}\left[r_{s+1}\right]$ requires attention at stage $s+1$ if

$$
\begin{aligned}
\operatorname{num}\left(w, \mathcal{B}_{\ell}\left[r_{s+1}\right]\lceil b)\right. & >\operatorname{num}\left(w, \mathcal{A}_{\leq \ell}[s]\right) \quad \text { and } \\
\operatorname{num}\left(u, \mathcal{B}_{\ell}\left[r_{s+1}\right]\lceil b)\right. & =0 .
\end{aligned}
$$

If no $b \in \mathcal{B}_{\ell}\left[r_{s+1}\right]$ requires attention at this stage, then declare the strategy of $G_{\ell}$ to be unrestrained, and move on to the action phase.

Otherwise, let $b_{0}$ be the $\leq_{\mathbb{N}}$-least $b \in \mathcal{B}_{\ell}\left[r_{s+1}\right]$ (i.e., the one of highest priority) that requires attention at this stage. We declare the strategy of $G_{\ell}$ to be restrained by $b_{0}$, unless the previous $\ell$-stage was restrained by $c$ for some $c<_{\mathbb{N}} b_{0}$, in which case we declare the strategy of $G_{\ell}$ to be unrestrained. (Notice that in this latter case, $c$ has stopped requiring attention at $s+1$, as $b_{0}$ is the $\leq_{\mathbb{N}}$-least element that requires attention. The idea here is that we want $G_{\ell}$ to have at least one unrestrained stage before being restrained by some element of lower priority.)

Action phase. If $G_{\ell}$ has the unrestrained strategy, then increase the 5-type of every minimal $w^{\infty}$ element of $\mathcal{A}_{\ell}[s]$ to $w$, except for one element which will remain with 5 -type $w^{\infty}$. (In doing this, we are taking one step towards building a 1-watom.)

Otherwise, if $G_{\ell}$ has a restrained strategy, do not change the 5-type of any element of $\mathcal{A}_{\ell}[s]$. (In doing this, we avoid building a 1-w-atom and add no new $w$ elements.)

Step 2. We have already defined $\mathfrak{t}_{5}^{\ell[s+1]}$ on all the elements of $\mathcal{A}_{\ell}[s]$. Extend $\mathcal{A}_{\ell}[s]$ to $\mathcal{A}_{\ell}[s+1]$ by splitting each minimal element $a$ of $\mathcal{A}_{\ell}[s]$ into a canonical $\mathfrak{t}_{5}^{\ell[s+1]}(a)$-full partition as described in Section 4

6.1. Verifications. We begin by proving that the construction does not get stuck in the strategy phase while waiting for one of the conditions (W1), (W2), (W3) to hold.

Lemma 6.3. Let $s+1$ be a stage at which $G_{\ell}$ is active. For each minimal element $b \in \mathcal{B}_{\ell}\left[r_{s}\right]$, there is some stage $r^{b} \geq r_{s}$ at which one of the conditions (W1)-(W3) is satisfied.

Proof. Fix a minimal element $b$ in $\mathcal{B}_{\ell}\left[r_{s}\right]$. Note that it is decidable during the construction whether or not each condition (W1)-(W3) holds for a given stage $r^{b}$. Suppose (W3) never occurs. If $e_{6} \notin \mathrm{dc} T_{5}^{\mathcal{B}_{\ell}}(b)$, then we will show that (W1) holds because $b$ bounds only finitely many elements of type $e_{6}$. Since $(D)_{4}=$ $\left\{e_{0}, e_{1}, e_{2}, e_{6}, e_{8}\right\}$, it can be verified using [HM, Section 6] that no element $\beta \in(D)_{4}$ satisfies $\beta>_{4} e_{6}$. Let $b=b_{0} \dot{V} \ldots \dot{\vee} b_{k}$ be a decomposition into 5-indecomposable elements in $\mathcal{B}_{\ell}$, so that by our hypothesis and Remark 2.14, $e_{6} \notin \operatorname{dc} T_{5}^{\mathcal{B}_{\ell}}\left(b_{i}\right)$ for each $i \leq k$. It follows from Definition 2.6 that each $b_{i}$ bounds only finitely many disjoint elements of 4 -bf-type $e_{6}$, so that $b$ only bounds finitely many such elements. Note that each $b_{i}$ in the decomposition of $b$ with 4-bf-type $e_{6}$ will have an exclusive 5 -bftype, since $e_{6} \notin \mathrm{dc} T_{5}^{\mathcal{B}_{\ell}}\left(b_{i}\right)$. Thus, there will be a stage $r^{b}$ sufficiently large such that 
each minimal element of $\mathcal{B}_{\ell}\left[r^{b}\right]\left\lceil b\right.$ with 4 -bf-type $e_{6}$ will be labeled with an exclusive 5 -bf-type, so that we will discover that (W1) holds. If $e_{6} \in \operatorname{dc} T_{5}^{\mathcal{B}_{\ell}}(b)$, then there are infinitely many disjoint $e_{6}$ elements in $\mathcal{B}_{\ell}\left\lceil b\right.$ because $e_{6}$ is the only 4-bf-type in $\left\{(\beta)_{4}: \beta \in D\right\}=\left\{e_{0}, e_{1}, e_{2}, e_{6}, e_{8}\right\}$ that is $\geq_{4} e_{6}$ (see [HM, Section 6]). Thus, there will be a sufficiently large stage $r^{b}$ with $\operatorname{num}\left(e_{6}, \mathcal{B}_{\ell}\left[r^{b}\right]\lceil b) \geq 2 \cdot \operatorname{num}\left(e_{6}, \mathcal{A}[s]\right)+2\right.$ and we will discover that (W2) holds.

We have shown that the construction always outputs a Boolean algebra $\mathcal{A}$, independently of the specifics of $z$ and $\left\{\mathcal{B}_{\ell}: \ell \in \omega\right\}$. We now show that if $\ell_{0}$ does exist, then $\mathcal{A} \neq \mathcal{B}_{\ell_{0}}$. This is necessarily true if (W3) holds at any stage; so, in what follows, we assume that this condition never holds.

Lemma 6.4. The algebras $\mathcal{A}_{\ell}$ have type $w^{\infty}$ and satisfy conditions (B1)-(B3)

Proof. For all $s$ and $\ell, \mathfrak{t}_{5}^{\ell[s]}(1)=w^{\infty}$, so each $\mathcal{A}_{\ell}$ has type $w^{\infty}$.

Suppose first that $\ell<\ell_{0}$. Then for all $s \geq \ell_{0}, z(s)>\ell$, hence $G_{\ell}$ is always inactive after stage $\ell_{0}$. Thus, the types of elements of $\mathcal{A}_{\ell}$ no longer change after stage $\ell_{0}$, so $\mathcal{A}_{\ell}$ will have densely many $w^{\infty}$ elements and just as many $w$ or $u$ elements as it had by stage $\ell_{0}$. Therefore, (B1) holds.

Suppose now that $\ell_{0}<\ell$. Then $G_{\ell}$ will be cancelled at some stage $s+1$, and hence $\mathcal{A}_{\ell}[s+1]$ has some $w^{\infty}$ elements, some $u$ elements and no $w$ elements. By Remark [5.2. $G_{\ell}$ will never be active after this stage, and hence it will always be inactive. $\mathcal{A}_{\ell}$ will end up having densely many $w^{\infty}$ elements, just as many $u$ elements as it had at stage $s+1$, and no $w$ elements. Thus, (B3) holds.

Suppose finally that $\ell=\ell_{0}$. Then there are infinitely many stages at which $z(s)=\ell_{0}$, and for every $s \geq \ell_{0}, z(s) \geq \ell_{0}$. Thus, $G_{\ell}$ is never cancelled, so it has no $u$-elements (which can be introduced only at the stage when $G_{\ell}$ is cancelled), and $G_{\ell}$ is active infinitely often. Since the 5-bf-types do not change when $G_{\ell}$ has the restrained strategy at $\ell$-stages, if $G_{\ell}$ has the restrained strategy at cofinitely many $\ell$-stages, then the 5 -bf-types of $\mathcal{A}_{\ell}$ will also not change at cofinitely many $\ell$-stages. In this case $\mathcal{A}_{\ell}$ will contain only finitely many elements of type $w$ and densely many elements of type $w^{\infty}$. If $G_{\ell}$ has the unrestrained strategy at infinitely many $\ell$-stages, $\mathcal{A}_{\ell}$ will have exactly one element of type $w^{\infty}$, because at each such stage there will be only one minimal element of $\mathcal{A}_{\ell}[s]$ left in $\mathcal{A}_{\ell}[s+1]$ with type $w^{\infty}$, as in Lemma 4.4

The following lemma shows how the number of $w$-elements in $\mathcal{A}[s]$ fluctuates. Keep in mind that for all $s$ and $\ell$,

$$
\operatorname{num}(w, \mathcal{A}[s])=\operatorname{num}\left(w, \mathcal{A}_{<\ell}[s]\right)+\operatorname{num}\left(w, \mathcal{A}_{\ell}[s]\right)+\operatorname{num}\left(w, \mathcal{A}_{>\ell}[s]\right) .
$$

Lemma 6.5. Let $s_{1}+1$ be an $\ell$-stage and let $s_{0}+1$ be the previous $\ell$-stage. Then

$$
\begin{aligned}
\operatorname{num}\left(w, \mathcal{A}_{<\ell}\left[s_{1}+1\right]\right) & =\operatorname{num}\left(w, \mathcal{A}_{<\ell}\left[s_{0}+1\right]\right)=\operatorname{num}\left(w, \mathcal{A}_{<\ell}\left[s_{1}\right]\right), \\
\operatorname{num}\left(w, \mathcal{A}_{>\ell}\left[s_{1}+1\right]\right) & =\operatorname{num}\left(w, \mathcal{A}_{>\ell}\left[s_{0}+1\right]\right)=0 \\
\operatorname{num}\left(w, \mathcal{A}_{\ell}\left[s_{1}+1\right]\right) & \leq \operatorname{num}\left(e_{6}, \mathcal{A}_{\ell}\left[s_{1}\right]\right) .
\end{aligned}
$$

Thus, $\operatorname{num}\left(w, \mathcal{A}\left[s_{1}+1\right]\right) \leq \operatorname{num}\left(e_{6}, \mathcal{A}\left[s_{1}\right]\right)$. Furthermore, if $G_{\ell}$ has a restrained strategy at $s_{1}+1$, then

$$
\operatorname{num}\left(w, \mathcal{A}\left[s_{1}+1\right]\right)=\operatorname{num}\left(w, \mathcal{A}\left[s_{0}+1\right]\right)=\operatorname{num}\left(w, \mathcal{A}_{\leq \ell}\left[s_{1}\right]\right) .
$$

Proof. Given that $s_{1}+1$ is an $\ell$-stage and $s_{0}+1$ is the previous $\ell$-stage, Remark 5.2 implies that for every stage $s$ with $s_{0}<s \leq s_{1}+1, z(s) \geq \ell$. Hence, every 
$\ell_{1}<\ell$ is inactive during these intermediate stages. Thus, $\operatorname{num}\left(w, \mathcal{A}_{<\ell}\left[s_{0}+1\right]\right)=$ $\operatorname{num}\left(w, \mathcal{A}_{<\ell}\left[s_{1}+1\right]\right)=\operatorname{num}\left(w, \mathcal{A}_{<\ell}\left[s_{1}\right]\right)$.

Each $\ell_{1}>\ell$ which is active before stage $s_{0}$, and has not yet been cancelled, will be cancelled at $s_{0}+1$. Therefore, $\operatorname{num}\left(w, \mathcal{A}_{>\ell}\left[s_{0}+1\right]\right)=0$. $\operatorname{Similarly,} \operatorname{num}\left(w, \mathcal{A}_{>\ell}\left[s_{1}+\right.\right.$ 1]) $=0$.

The third inequality follows from the fact that every minimal element in $\mathcal{A}_{\ell}\left[s_{1}\right]$ of type $e_{6}$ has, in $\mathcal{A}_{\ell}\left[s_{1}+1\right]$, at most one minimal element of type $w$ below.

If $G_{\ell}$ has a restrained strategy at $s_{1}+1, \operatorname{num}\left(w, \mathcal{A}_{<\ell}\left[s_{1}\right]\right)=\operatorname{num}\left(w, \mathcal{A}_{<\ell}\left[s_{1}+1\right]\right)$. Since $\ell$ is not active between stages $s_{0}+2$ and $s_{1}$, it follows that $\operatorname{num}\left(w, \mathcal{A}_{\leq \ell}\left[s_{0}+1\right]\right)$ $=\operatorname{num}\left(w, \mathcal{A}_{\leq \ell}\left[s_{1}\right]\right)$. So, $\operatorname{num}\left(w, \mathcal{A}\left[s_{1}+1\right]\right)=\operatorname{num}\left(w, \mathcal{A}\left[s_{0}+1\right]\right)=\operatorname{num}\left(w, \mathcal{A}_{\leq \ell}\left[s_{1}\right]\right)$.

Observation 6.6. Recall that we are assuming that the algebra $\mathcal{B}_{\ell}$ does not satisfy (W3). The 5-approximation of $\mathcal{B}_{\ell}$ need not respect the properties (D1)-(D4) from Section 4, however, there are several observations that do hold about the 5 -approximation of $\mathcal{B}_{\ell}$.

Using [HM, Section 6] and $\left\{(\beta)_{4}: \beta \in D\right\}=\left\{e_{0}, e_{1}, e_{2}, e_{6}, e_{8}\right\}$, one can verify that $e_{6} \nless_{4} \beta$ for any $\beta \in(D)_{4}$, so by Lemma 2.19 if $e_{6} \leq_{4} \beta_{0}+\ldots+\beta_{k}$ and each $\beta_{i} \in$ $(D)_{4}$, then $\beta_{i}=e_{6}$ for some $i \leq k$. If $b \in \mathcal{B}_{\ell}[s]$ with $\mathfrak{t}_{4}^{\mathcal{B}_{\ell}[s]}(b)=e_{6}$, then at each later stage $t$, there is some $b_{1} \leq b$ which is minimal in $\mathcal{B}_{\ell}[t]$ with $\mathfrak{t}_{4}^{\mathcal{B}_{\ell}[s]}(b)=e_{6}$. It follows that $\operatorname{num}\left(e_{6}, \mathcal{B}_{\ell}[t]\lceil b)\right.$ is non-decreasing in $t$ and $\operatorname{num}\left(e_{6}, \mathcal{B}_{\ell}[t]\lceil b) \leq \operatorname{num}\left(e_{6}, \mathcal{B}_{\ell}\lceil b)\right.\right.$ for each $b \in \mathcal{B}_{\ell}$.

Suppose that $\sigma=\beta_{0}+\ldots+\beta_{k} \in \mathbf{I N V}_{5}$ and $(\sigma)_{4}=e_{6}$. As we pointed out in the proof of Theorem 4.1(3),$\langle D\rangle_{4}=\left\{e_{0}, e_{1}, e_{2}, e_{6}, e_{8}\right\}=\mathrm{dc} w^{\infty}$. We now show that $w^{\infty} \leq_{5} \sigma$ if and only if $\operatorname{dc} \sigma \subseteq(D)_{4}$ and $\left(\beta_{i}\right)_{4} \in(D)_{4}$ for each $i$. The implication $(\Rightarrow)$ follows immediately from Lemma 2.19 . For the reverse direction, if each $\left(\beta_{j}\right)_{4} \in(D)_{4}$ and $e_{6}=\left(\beta_{0}\right)_{4}+\ldots+\left(\beta_{k}\right)_{4}$, then some $\left(\beta_{i}\right)_{4}=e_{6}$ (by the previous paragraph), and since $\operatorname{dc} \beta_{i} \subseteq \operatorname{dc} \sigma \subseteq(D)_{4}=w^{\infty}$, it follows from Definition 2.7 that $w^{\infty} \leq_{5} \beta_{i}$. So, $w^{\infty} \leq_{5} \sigma$ by Lemma 2.19. As a consequence, if $\sigma<_{5} w^{\infty}$, then either $\operatorname{dc} \sigma \nsubseteq(D)_{4}$ or there is some $\left(\beta_{i}\right)_{4} \notin(D)_{4}$.

If $\mathfrak{t}_{5}^{\mathcal{B}_{\ell}[s]}(b)=w$, but at a later stage $t, \mathfrak{t}_{5}^{\mathcal{B}_{\ell}[t]}(b)>_{5} w$, then there is a partition $b=b_{0} \dot{V} \ldots \dot{\vee} b_{k}$, where each $b_{i} \in \mathcal{B}_{\ell}[t]$ is minimal, $\mathfrak{t}_{5}^{\mathcal{B}_{\ell}[t]}\left(b_{i}\right)=\beta_{i}$ and $w<_{5}$ $\beta_{0}+\ldots+\beta_{k}$. It follows from Lemma 2.19 that $w \leq_{5} \beta_{i}$ for some $i$ and is an exclusive 5 -bf-type because $e_{6} \notin \mathrm{dc} \beta_{i}$. In fact, $w<_{5} \beta_{i}$ because if $w \equiv_{5} \beta_{i}$, then since $w \triangleleft_{5} \beta_{j}$ for each $j \neq i$ we would have $w \equiv_{5} \beta_{0}+\ldots+\beta_{k}$ by several applications of Lemma 2.20. Thus, for all stages $t^{\prime} \geq t, b$ will bound a minimal element in $\mathcal{B}_{\ell}\left[t^{\prime}\right]$ whose 5-bf-type $>_{5} w$, and whose 5-bf-type must therefore be $u$ (as we noted at the beginning of Section 4).

Finally, since $e_{6} \triangleleft_{4} \beta$ for each $\beta \in \mathbf{B F}_{4}$ (using [HM, Section 6]), it follows from several applications of Lemma 2.20 that $e_{6} \equiv_{4} e_{6}+\rho$ for each $\rho \in \mathbf{I N V}_{4}$. As a consequence, if $\operatorname{num}\left(e_{6}, \mathcal{B}_{\ell}\lceil b)=\infty\right.$, then $T_{4}^{\mathcal{B}_{\ell}}(b)=e_{6}$.

Lemma 6.7. Suppose there exists an $\ell_{0}$ with $z(s)=\ell_{0}$ for infinitely many $s$. Then one of the following holds:

(1) There exist a $b_{0} \in \mathcal{B}_{\ell_{0}}$ and some $s_{0}$ such that for every $\ell_{0}$-stage $s \geq s_{0}, G_{\ell_{0}}$ is restrained by $b_{0}$. In this case, we win by (S1).

(2) There is no such $b_{0}$, and we win by (S2). 
Proof. Suppose first that there exists a $b_{0}$ as in (1). Then $\operatorname{num}\left(w, \mathcal{B}_{\ell_{0}}\left[r_{s_{0}+1}\right]\left\lceil b_{0}\right)>\right.$ $\operatorname{num}\left(w, \mathcal{A}_{\leq \ell_{0}}\left[s_{0}\right]\right)$. By Lemma 6.5 the number of $w$ elements does not change between successive $\ell_{0}$-stages, and since $G_{\ell_{0}}$ is restrained by $b_{0}$ at all $\ell_{0}$-stages $\geq s_{0}$ and a $w$ element can only bound a single minimal $w$ element (by Lemma $4.1(\overline{5 b})$ ), no new $w$ elements are added at $\ell_{0}$-stages. $\operatorname{Son} \operatorname{num}\left(w, \mathcal{A}_{\leq \ell_{0}}\left[s_{0}\right]\right)=\operatorname{num}(w, \mathcal{A})$. Since $b_{0}$ requires attention at every $\ell_{0}$-stage $s \geq s_{0}$, we have that $\operatorname{num}\left(u, \mathcal{B}_{\ell_{0}}\left[r_{s}\right]\left\lceil b_{0}\right)=0\right.$ at all these stages. This implies that no $w$ element in $\mathcal{B}_{\ell_{0}}\left[r_{s_{0}+1}\right]\lceil b$ can change its 5-bf-type, because, by Observation 6.6. some new $u$ element would show up. Hence $\operatorname{num}\left(w, \mathcal{B}_{\ell_{0}}\left\lceil b_{0}\right) \geq \operatorname{num}\left(w, \mathcal{B}_{\ell_{0}}\left[r_{s_{0}+1}\right]\left\lceil b_{0}\right)\right.\right.$. We conclude that

$$
\operatorname{num}\left(w, \mathcal{B}_{\ell_{0}}\right) \geq \operatorname{num}\left(w, \mathcal{B}_{\ell_{0}}\left[r_{s_{0}+1}\right]\left\lceil b_{0}\right)>\operatorname{num}\left(w, \mathcal{A}_{\leq \ell_{0}}\left[s_{0}\right]\right)=\operatorname{num}(w, \mathcal{A}),\right.
$$

and thus (S1).

Suppose now there is no such $b_{0}$. We first prove that there are infinitely many unrestrained $\ell_{0}$-stages, and hence that $\mathcal{A}_{\ell_{0}}$ is a 1 - $w$-atom. Note that if $s_{0}$ and $s_{1}$ are consecutive $\ell_{0}$-stages at which $G_{\ell_{0}}$ is restrained (say, by $c_{0}$ and $c_{1}$ respectively), then necessarily $c_{0} \geq_{\mathbb{N}} c_{1}$ (because if $c_{0}$ is less than the least $c$ that requires attention at stage $s_{1}$, namely, $c_{1}$, then $G_{\ell_{0}}$ would have an unrestrained strategy at $s_{1}$ ). Therefore, if $G_{\ell_{0}}$ is restrained from some $\ell_{0}$-stage on, then from some point on it will be restrained by the same $c$. But we are assuming that (1) does not hold. This implies that there are infinitely many unrestrained $\ell_{0}$-stages. Then, by Lemma 4.4 . $\mathcal{A}_{\ell_{0}}$ is a 1 -w-atom.

Next, we prove that there is no $b \in \mathcal{B}_{\ell_{0}}$ such that $\mathcal{B}_{\ell_{0}}\lceil b$ is a 1 -w-atom. At the same time, by induction on $b \in \mathbb{N}$, we show that for each $b$ there is a stage after which $G_{\ell_{0}}$ is never restrained by $b$. Suppose this is the case for all $c<_{\mathbb{N}} b$. Let $s_{0}$ be an $\ell_{0}$-stage after which $G_{\ell_{0}}$ is never restrained by any $c<_{\mathbb{N}} b$. If $G_{\ell_{0}}$ is ever restrained by $b$ at some $\ell_{0}$-stage $s \geq s_{0}$, then at some stage $s_{1}>s$ it has to stop requiring attention since we are assuming (11) does not hold. This means that either $\operatorname{num}\left(w, \mathcal{B}_{\ell_{0}}\left[r_{s_{1}}\right]\lceil b) \leq \operatorname{num}\left(w, \mathcal{A}_{\leq \ell}\left[s_{1}-1\right]\right)\right.$ or $\operatorname{num}\left(u, \mathcal{B}_{\ell_{0}}\left[r_{s_{1}}\right]\lceil b)>0\right.$. In the former case, we have that $\operatorname{num}\left(w, \mathcal{A}_{\leq \ell}\left[s_{1}-1\right]\right)=\operatorname{num}\left(w, \mathcal{A}_{\leq \ell}[s]\right)$, by using Lemma 6.5 at the $\ell_{0}$-stages between $s$ and $s_{1}$. So, $\operatorname{num}\left(w, \mathcal{B}_{\ell_{0}}\left[r_{s}\right]\lceil\bar{b})>\operatorname{num}\left(w, \mathcal{B}_{\ell_{0}}\left[r_{s_{1}}\right]\lceil b)\right.\right.$. But the only way the number of $w$ elements can decrease is if some of them increase their 5-bf-type and elements of exclusive type $>_{5} w$ appear (by Observation 6.6). Therefore, in either case, some $>_{5} w$ element had to appear below $b$ at stage $s_{1}$. Since the 5-bf-type elements never decrease, $b$ will never again require attention, and is not a $1-w$-atom. Thus, there is a stage after which $G_{\ell_{0}}$ is never again restrained by $b$, and $b$ never again requires attention.

Assume that $b$ is a 1 - $w$-atom. By Definition 4.3 of a 1 - $w$-atom, $T_{5}^{\mathcal{B}_{\ell_{0}}}(b)=w^{\infty}$, and so $\operatorname{num}\left(e_{6}, \mathcal{B}_{\ell_{0}}\lceil b)=\infty\right.$. For any partition $b=b_{0} \dot{V} \ldots \dot{\vee} b_{k}$, there is some $i$ for which $\operatorname{num}\left(e_{6}, \mathcal{B}_{\ell_{0}}\left\lceil b_{i}\right)=\infty\right.$, so that $T_{4}^{\mathcal{B}_{\ell_{0}}}\left(b_{i}\right)=e_{6}$ (by the final paragraph of Observation 6.6), and thus $T_{5}^{\mathcal{B}_{\ell_{0}}}\left(b_{i}\right)=w^{\infty}$ (from part (ii) of Definition 4.3). If $\mathfrak{t}_{5}^{\mathcal{B}_{\ell_{0}}[s]}(b)<_{5} w^{\infty}$ at any stage $s$ after $b$ first enters the 5 -approximation for $\mathcal{B}_{\ell_{0}}$, then by the second paragraph of Observation 6.6. either $\operatorname{dc}_{5} \mathfrak{B}_{5}{ }^{\mathcal{\ell}_{0}}[s](b) \not \subset(D)_{4}=\mathrm{dc} w^{\infty}$, so that there is a $\beta \in \mathfrak{t}_{5}^{\mathcal{B}_{\ell_{0}}[s]}(b)-(D)_{4}$ or $\mathfrak{t}_{5}^{\mathcal{B}_{\ell_{0}}[s]}(b)=\tau+\beta^{*}$ where $\left(\beta^{*}\right)_{4} \notin(D)_{4}$. However, in either case since $b$ splits into a $\mathfrak{t}_{5}^{\mathcal{B}_{\ell_{0}}[s]}(b)$-full partition in $B_{\ell_{0}}[s]$ there is a $b^{\prime}<b$ in $B_{\ell_{0}}[s]$ with $\mathfrak{t}_{4}^{\mathcal{B}_{\ell_{0}}[s]}\left(b^{\prime}\right) \notin(D)_{4}$. In fact, there must be a minimal $b^{\prime}<b$ in $B_{\ell_{0}}[s]$ with $\mathfrak{t}_{4}^{\mathcal{B}_{\ell_{0}}[s]}\left(b^{\prime}\right) \notin(D)_{4}$ : suppose $b^{\prime}<b$ in $B_{\ell_{0}}[s]$ with $\mathfrak{t}_{4}^{\mathcal{B}_{\ell_{0}}[s]}\left(b^{\prime}\right) \notin(D)_{4}$ and let $b^{\prime}=c_{0} \dot{\vee} \ldots \dot{\vee} c_{k}$ be a partition into minimal elements in $B_{\ell_{0}}[s]$, then there is some 
$c_{i}$ with $\mathfrak{t}_{4}^{\mathcal{B}_{\ell_{0}}[s]}\left(b^{\prime}\right) \leq_{4} \mathfrak{t}_{4}^{\mathcal{B}_{\ell_{0}}[s]}\left(c_{i}\right)$ so that $\mathfrak{t}_{4}^{\mathcal{B}_{\ell_{0}}[s]}\left(c_{i}\right) \notin(D)_{4}$ as well. Therefore, we must have $\mathfrak{t}_{5}^{\mathcal{B}_{\ell_{0}}[s]}(b)=w^{\infty}$ for all stages $s$ after $b$ that first enter the 5 -approximation for $\mathcal{B}_{\ell_{0}}$. So, if $b=b_{0} \dot{\vee} \ldots \dot{\vee} b_{k}$ is a partition in $\mathcal{B}_{\ell_{0}}[s]$ into minimal elements, there must be some $i$ with $\mathfrak{t}_{5}^{\mathcal{B}_{\ell_{0}}[s]}\left(b_{i}\right) \leq_{5} w^{\infty}$, but since $\mathfrak{t}_{5}^{\mathcal{B}_{\ell_{0}}[s]}(b)=w^{\infty}$, we must have $\mathfrak{t}_{5}^{\mathcal{B}_{\ell_{0}}[s]}\left(b_{i}\right)=w^{\infty}$. Moreover, each $c<b$ which is an $e_{6}$ element with exclusive 5-bftype in $\mathcal{B}_{\ell_{0}}[s]$ must have $\mathfrak{t}_{5}^{\mathcal{B}_{\ell_{0}}[s]}(c)=w$, because all exclusive 5 -bf-types are $\geq_{5} w$ and $b$ bounds no elements whose 5 -bf-type is $>_{5} w$ by part (ii) of Definition 4.3. Let $s_{1}$ be an $\ell_{0}$-stage beyond which $b$ never again restrains $G_{\ell_{0}}$ and let $b_{1} \leq b$ in $\mathcal{B}_{\ell_{0}}\left[s_{1}\right]$ be a minimal $w^{\infty}$ element for which $r^{b_{1}}$ is defined by (W2). It follows that $\operatorname{num}\left(e_{6}, \mathcal{B}_{\ell_{0}}\left[r_{s_{1}}\right]\lceil b) \geq 2 \cdot \operatorname{num}\left(e_{6}, \mathcal{A}\left[s_{1}-1\right]\right)+2\right.$. Let $d \in \mathcal{B}_{\ell_{0}}\left[s_{1}\right]\lceil b$ be such that

$$
\begin{aligned}
\operatorname{num}\left(e_{6}, \mathcal{B}_{\ell_{0}}\left[r_{s_{1}}\right]\lceil d)\right. & >\operatorname{num}\left(e_{6}, \mathcal{A}\left[s_{1}-1\right]\right) \quad \text { and } \\
\operatorname{num}\left(e_{6}, \mathcal{B}_{\ell_{0}}\left[r_{s_{1}}\right]\lceil b-d)\right. & >\operatorname{num}\left(e_{6}, \mathcal{A}\left[s_{1}-1\right]\right) .
\end{aligned}
$$

If both $e_{6} \in \operatorname{dc} T_{5}^{\mathcal{B}_{\ell_{0}}}(d)$ and $e_{6} \in \operatorname{dc} T_{5}^{\mathcal{B}_{\ell}}(b-d)$, then it follows from the first paragraph of Observation 6.6 that $\operatorname{num}\left(e_{6}, d\right)=\operatorname{num}\left(e_{6}, b-d\right)=\infty$, contradicting our assumption that $\mathcal{B}_{\ell_{0}}\left\lceil b\right.$ is a 1-w-atom. Suppose $e_{6} \notin \mathrm{dc} T_{5}^{\mathcal{B}_{\ell_{0}}}(d)$, and thus that there is an $\ell_{0}$-stage $s>s_{1}$ with $e_{6} \notin \mathrm{dc} \mathfrak{t}_{5}^{\mathcal{B}_{\ell_{0}}\left[r_{s}\right]}(d)$. Let $s_{3}>s_{1}$ be the first such stage and note that $\operatorname{num}\left(e_{6}, \mathcal{B}_{\ell_{0}}\left[r_{s_{3}}\right]\lceil d)=\operatorname{num}\left(w, \mathcal{B}_{\ell_{0}}\left[r_{s_{3}}\right]\lceil d)\right.\right.$, since each minimal $e_{6}$ element below $d$ is an exclusive 5 -bf-type. We will show that $b$ requires attention at $s_{3}$, in contradiction to our assumption that this never happens beyond $s_{1}$. To establish this, we will first show that for each $\ell_{0}$-stage $s$ with $s_{1} \leq s<s_{3}$,

$$
\operatorname{num}\left(e_{6}, \mathcal{A}[s-1]\right)<\operatorname{num}\left(e_{6}, \mathcal{B}_{\ell_{0}}\left[r_{s}\right]\lceil d) .\right.
$$

This is true at $s=s_{1}$ by hypothesis. Let $s^{\prime}$ be the previous $\ell_{0}$-stage to $s$, so that $s_{1} \leq s^{\prime}<s<s_{3}$. Since $s_{3}$ is the first $\ell_{0}$-stage with $e_{6} \notin \mathrm{dc} \mathfrak{t}_{5}^{\mathcal{B}_{\ell_{0}}\left[r_{s_{3}}\right]}(d)$, for each $r_{s_{1}} \leq t \leq r_{s}$ we have $e_{6} \in \mathrm{dc} \mathfrak{t}_{5}^{\mathcal{B}_{\ell_{0}}[t]}(d)$, so that if $d=d_{0} \dot{V} \ldots \dot{V} d_{k}$ is a partition into minimal elements of $\mathcal{B}_{\ell_{0}}[t]$, then there is some $i$ with $e_{6} \in \operatorname{dct}_{5}^{\mathcal{B}_{\ell_{0}}[t]}\left(d_{i}\right)$. Thus, there is a minimal element $d_{2}$ in $\mathcal{B}_{\ell_{0}}\left[r_{s}\right]$ with $e_{6} \in \mathrm{dct}_{5}^{\mathcal{B}_{\ell_{0}}\left[r_{s}\right]}\left(d_{2}\right)$; furthermore, $d_{2}$ must lie below some minimal $d_{1} \in \mathcal{B}_{\ell_{0}}\left[r_{s^{\prime}}\right]$ for which $e_{6} \in \operatorname{dct}_{5} \mathfrak{B}_{\ell_{0}}[t]\left(d_{1}\right)$ for all $r_{s^{\prime}} \leq t \leq r_{s}$. Fix $r_{s^{\prime}} \leq t<r_{s}$ and let $c<d_{1}$ be minimal in $\mathcal{B}_{\ell_{0}}[t]$ for which $e_{6} \in \operatorname{dct}_{5}^{\mathcal{B}_{\ell_{0}}[t+1]}(c)$ and let $c=c_{0} \dot{V} \ldots \dot{V} c_{k}$ be minimal in $\mathcal{B}_{\ell_{0}}[t+1]$. We must have some $c_{i}$ with $\mathfrak{t}_{4}^{\mathcal{B}_{\ell_{0}}[t+1]}\left(c_{i}\right)=e_{6}$ by part (2) of Definition 3.2 (otherwise there would be an element below $c$ whose 4 -bf-type is not in $\left.(D)_{4}\right)$, so that $\mathfrak{t}_{4}^{\mathcal{B}_{0}}{ }^{[t+1]}(c)=e_{6}$ as well by the final paragraph of Observation 6.6. It follows that there are at least two new minimal elements of type $e_{6}$ below $c$ in $\mathcal{B}_{\ell_{0}}[t+1]$ (one is required by part (1) of Definition 3.2 and the rest by part $(2))$, and so $\operatorname{num}\left(e_{6}, \mathcal{B}_{\ell_{0}}[t]\left\lceil d_{1}\right)<\operatorname{num}\left(e_{6}, \mathcal{B}_{\ell_{0}}[t+1]\left\lceil d_{1}\right)\right.\right.$. Since the number of elements of type $e_{6}$ is increasing for each $r_{s^{\prime}} \leq t<r_{s}$ and $d_{1}$ is minimal in $\mathcal{B}_{\ell_{0}}\left[r_{s^{\prime}}\right], r^{d_{1}}$ will be defined by (W2), and so

$$
\operatorname{num}\left(e_{6}, \mathcal{A}[s-1]\right)<\operatorname{num}\left(e_{6}, \mathcal{B}_{\ell_{0}}\left[r_{s}\right]\left\lceil d_{1}\right) \leq \operatorname{num}\left(e_{6}, \mathcal{B}_{\ell_{0}}\left[r_{s}\right]\lceil d) .\right.\right.
$$

Let $s_{2}$ be the last $\ell_{0}$-stage before $s_{3}$; then

$$
\operatorname{num}\left(e_{6}, \mathcal{A}\left[s_{2}-1\right]\right)<\operatorname{num}\left(e_{6}, \mathcal{B}_{\ell_{0}}\left[r_{s_{2}}\right]\lceil d) \leq \operatorname{num}\left(e_{6}, \mathcal{B}_{\ell_{0}}\left[r_{s_{3}}\right]\lceil d) .\right.\right.
$$

By Lemma 6.5,

$$
\operatorname{num}\left(w, \mathcal{A}_{\leq \ell_{0}}\left[s_{3}-1\right]\right)=\operatorname{num}\left(w, \mathcal{A}\left[s_{2}\right]\right) \leq \operatorname{num}\left(e_{6}, \mathcal{A}\left[s_{2}-1\right]\right) .
$$


Hence $\operatorname{num}\left(w, \mathcal{A}_{\leq \ell_{0}}\left[s_{3}-1\right]\right)<\operatorname{num}\left(w, \mathcal{B}_{\ell_{0}}\left[r_{s_{3}}\right]\lceil b)\right.$ and $b$ requires attention at stage $s_{3}$ in contradiction to our assumption that $b$ never requires attention after $s_{1}$.

This proves that $G_{\ell_{0}}$ is satisfied and hence finishes the proof of Proposition 6.2 and Theorem 6.1

6.2. Main theorem. We now come to the main result in this paper.

Theorem 6.8. There is a low 5 Boolean algebra that is not $0^{(7)}$-isomorphic to any computable Boolean algebra.

Proof. We relativize Theorem 6.1 to $0^{(4)}$ : There exists a $5-0^{(5)}$-approximableBoolean algebra $\mathcal{A}$ which is not $0^{(7)}$-isomorphic to any $4-0^{(4)}$-approximable algebra. Recall that every computable presentation of a Boolean algebra is $4-0^{(4)}$ approximable, hence $\mathcal{A}$ is not $0^{(7)}$-isomorphic to any computable Boolean algebra. By Theorem 2.11, there is a low ${ }_{5}$ copy $\mathcal{B}$ of $\mathcal{A}$ via an isomorphism that is computable in $0^{(5)}$. If $\mathcal{B}$ were $0^{(7)}$-isomorphic to a computable Boolean algebra, then $\mathcal{A}$ would be too.

Remark 6.9. The Boolean algebra $\mathcal{A}$ constructed in the previous proof is isomorphic to a computable one. The reason is that the Boolean algebra $\mathcal{A}$ (that is, $\mathcal{A}_{\langle e, i\rangle}$ ) constructed in Proposition 6.2 can be shown to be isomorphic to either

$$
\bigoplus_{i=1}^{k} \mathcal{A}_{w} \oplus \sum_{i=1}^{\omega}\left(\mathcal{A}_{u} \oplus \mathcal{A}_{w^{\infty}}\right) \quad \text { or } \quad \mathcal{A}_{1 w} \oplus \sum_{i=1}^{\omega}\left(\mathcal{A}_{u} \oplus \mathcal{A}_{w^{\infty}}\right),
$$

when $\ell_{0}$ exists, and to

$$
\sum_{i=1}^{\omega}\left(\mathcal{A}_{u} \oplus \mathcal{A}_{w} \oplus \mathcal{A}_{w^{\infty}}\right)
$$

when it does not, where $\mathcal{A}_{u}$ is the unique isomorphism type of 5-bf-type $u$, namely, $\operatorname{Int}\left(\omega^{2}+\eta\right) ; \mathcal{A}_{w}$ is the unique isomorphism type of 5-bf-type $w$ which satisfies properties (D1)-(D4) of Section 4. namely, the interval algebra $\operatorname{Int}\left(\omega^{3}+\eta\right) ; \mathcal{A}_{w^{\infty}}$ is the isomorphism type of the Boolean algebra of 5-bf-type $w^{\infty}$, which satisfies (D1)-(D4) and has the property that the 5-bf-types never increase, namely, $\operatorname{Int}\left(\left(\omega^{2}+1+\eta\right) \cdot \eta\right)$; and $\mathcal{A}_{1 w}$ is the 1 - $w$-atom which satisfies (D1)-(D4), namely, $\operatorname{Int}\left(\left(\omega^{3}+\eta\right) \cdot \omega\right)$. All these algebras are computably presentable.

Furthermore, $0^{(7)}$ can go through the construction in the proof of Proposition 6.2 and decide how the requirements are satisfied (recall that the proof given there is later relativized to $0^{(5)}$ ), so $0^{(7)}$ can find an isomorphism between $\mathcal{A}_{\langle e, i\rangle}$ and one of these computable algebras. However, $0^{(7)}$ does not know if $\ell_{0}$ exists, so it cannot uniformly compute these isomorphisms. But $0^{(8)}$ can. One can then show that the relativization of the Boolean algebra $\mathcal{A}=\sum_{e, i \in \omega} \mathcal{A}_{\langle e, i\rangle}$ constructed in the proof of Theorem 6.1 is $0^{(8)}$-isomorphic to the following computable Boolean algebra:

$$
\sum_{j=1}^{\omega}\left(\mathcal{A}_{w} \oplus \mathcal{A}_{1 w} \oplus \sum_{i=1}^{\omega}\left(\mathcal{A}_{u} \oplus \mathcal{A}_{w}\right) \oplus \sum_{i=1}^{\omega}\left(\mathcal{A}_{u} \oplus \mathcal{A}_{w} \oplus \mathcal{A}_{w}\right)\right) .
$$

\section{ACKNOWLEDGEMENT}

The authors would like to thank the anonymous referee for a detailed report. 


\section{REFERENCES}

[AK00] C. J. Ash and J. Knight, Computable structures and the hyperarithmetical hierarchy, Studies in Logic and the Foundations of Mathematics, vol. 144, North-Holland Publishing Co., Amsterdam, 2000. MR1767842 (2001k:03090)

[Ala04] P. E. Alaev, Computable homogeneous Boolean algebras and a metatheorem (Russian, with Russian summary), Algebra Logika 43 (2004), no. 2, 133-158, 256, DOI 10.1023/B:ALLO.0000020844.03135.a6; English transl., Algebra Logic 43 (2004), no. 2, 73-87. MR2072567 (2005d:03082)

[DJ94] Rod Downey and Carl G. Jockusch, Every low Boolean algebra is isomorphic to a recursive one, Proc. Amer. Math. Soc. 122 (1994), no. 3, 871-880, DOI 10.2307/2160766. MR $1203984(95 \mathrm{a}: 03044)$

[HM] Kenneth Harris and Antonio Montalbán, On the $n$-back-and-forth types of Boolean algebras. Trans. Amer. Math. Soc. 364 (2012), no. 2, 827-866. MR2846355

[Kec95] Alexander S. Kechris, Classical descriptive set theory, Graduate Texts in Mathematics, vol. 156, Springer-Verlag, New York, 1995. MR1321597 (96e:03057)

[KS00] Julia F. Knight and Michael Stob, Computable Boolean algebras, J. Symbolic Logic 65 (2000), no. 4, 1605-1623, DOI 10.2307/2695066. MR1812171(2001m:03086)

[Mon] Antonio Montalbán, Notes on the jump of a structure, Mathematical theory and computational practice, Lecture Notes in Comput. Sci., vol. 5635, Springer, Berlin, 2009, pp. 372-378, DOI 10.1007/978-3-642-03073-4_38. MR2545911 (2011h:03059)

[Mon89] Sabine Koppelberg, Handbook of Boolean algebras. Vol. 1, North-Holland Publishing Co., Amsterdam, 1989. Edited by J. Donald Monk and Robert Bonnet. MR991565 (90k:06002)

[Thu95] John J. Thurber, Every low 2 Boolean algebra has a recursive copy, Proc. Amer. Math. Soc. 123 (1995), no. 12, 3859-3866, DOI 10.2307/2161917. MR1283564 (96b:03047)

Department of Mathematics, University of Michigan, Ann Arbor, Michigan 48109

E-mail address: kaharri@umich.edu

Department of Mathematics, University of Chicago, Chicago, Illinois 60637

E-mail address: antonio@math.uchicago.edu

$U R L$ : www.math.uchicago.edu/ antonio 